\title{
Cantor spectrum of graphene in magnetic fields
}

\author{
Simon Becker ${ }^{1}$ - Rui Han ${ }^{2}$. \\ Svetlana Jitomirskaya ${ }^{3}$
}

Received: 8 May 2018 / Accepted: 13 June 2019 / Published online: 17 August 2019

(C) The Author(s) 2019

\begin{abstract}
We consider a quantum graph as a model of graphene in magnetic fields and give a complete analysis of the spectrum, for all constant fluxes. In particular, we show that if the reduced magnetic flux $\Phi / 2 \pi$ through a honeycomb is irrational, the continuous spectrum is an unbounded Cantor set of Lebesgue measure zero.
\end{abstract}

\section{Introduction}

Graphene is a two-dimensional material that consists of carbon atoms at the vertices of a hexagonal lattice. Its experimental discovery, unusual properties, and applications led to a lot of attention in physics, see e.g. [44]. Electronic properties of graphene have been extensively studied rigorously in the absence of magnetic fields [20-22,39].

Simon Becker

simon.becker@damtp.cam.ac.uk

Rui Han

rui.han@math.gatech.edu

Svetlana Jitomirskaya

szhitomi@math.uci.edu

1 DAMTP, University of Cambridge, Wilberforce Rd, Cambridge CB3 OWA, UK

2 School of Math, Georgia Institute of Technology, Atlanta, USA

3 Department of Mathematics, University of California, Irvine, USA 
Magnetic properties of graphene have also attracted strong interest in physics (e.g. [27,55]). The purpose of this paper is to provide for the first time an analysis of the spectrum of honeycomb structures in magnetic fields with constant flux.

The fact that magnetic electron spectra have fractal structures was first predicted by Azbel [3] and then numerically observed by Hofstadter [31], for the Harper's model. The scattering plot of the electron spectrum as a function of the magnetic flux is nowadays known as Hofstadter's butterfly. Verifying such results experimentally has been restricted for a long time due to the extraordinarily strong magnetic fields required. Only recently, self-similar structures in the electron spectrum in graphene have been observed [15,17,23,25].

With this work, we provide a rigorous foundation for self-similarity by showing that for irrational fluxes, the electron spectrum of a model of graphene is a Cantor set. We say $A$ is a Cantor set if it is closed, nowhere dense and has no isolated points (so compactness not required). The Schrödinger operator $H^{B}$ we study, see (3.7), is defined on a metric honeycomb graph ${ }^{1}$ and is a direct sum, over all edges $\vec{e}$ of the graph, of Schrödinger operators

$$
H_{\vec{e}}^{B}=\left(-i \partial_{x}-A_{\vec{e}}\right)^{2}+V_{\vec{e}}
$$

with magnetic potential $A_{\vec{e}}$, describing a constant magnetic field, and potential $V_{\vec{e}} \in L^{2}(\vec{e})$. We write $\sigma^{\Phi}, \sigma_{\text {cont }}^{\Phi}, \sigma_{\text {ess }}^{\Phi}$ for the (continuous, essential) spectra of $H^{B}$ and set $H^{D}$ to be the Dirichlet operator (no magnetic field) defined in (2.14) (2.11), and denote by $\sigma\left(H^{D}\right)$ its spectrum. Let $\sigma_{p}^{\Phi}$ be the collection of eigenvalues of $H^{B}$. Then we have the following description of the topological structure and point/continuous decomposition of the spectrum

Theorem 1 For any symmetric Kato-Rellich potential $V_{\vec{e}} \in L^{2}(\vec{e})$ we have

(1) $\sigma^{\Phi}=\sigma_{\text {ess }}^{\Phi}$,

(2) $\sigma_{p}^{\Phi}=\sigma\left(H^{D}\right)$,

(3) $\sigma_{\text {cont }}^{\Phi}$ is

- a Cantor set of measure zero for $\Phi \notin 2 \pi \mathbb{Q}$,

- a countable union of disjoint intervals for $\Phi \in 2 \pi \mathbb{Q}$,

(4) $\sigma_{p}^{\Phi} \cap \sigma_{\text {cont }}^{\Phi}=\emptyset$ for $\Phi \notin 2 \pi \mathbb{Z}$,

(5) the Hausdorff dimension $\operatorname{dim}_{H}\left(\sigma^{\Phi}\right) \leq 1 / 2$ for generic ${ }^{2} \Phi$.

Thus for irrational flux, the spectrum is a zero measure Cantor set plus a countable collection of flux-independent isolated eigenvalues, each of infinite

\footnotetext{
1 Schrödinger operators defined on metric graphs are also called quantum graphs.

2 In this paper, "generic" refers to a dense $G_{\delta}$ set. Recently, a stronger continuity of spectra statement was proved in [33], which combined with Lemma 4.3 allowed the authors to extend the Hausdorff dimension statement to all irrational $\Phi$.
} 
multiplicity, while for rational flux the Cantor set is replaced by a countable union of intervals.

Furthermore, we can also describe the spectral decomposition of $H^{B}$.

\section{Theorem 2 For any symmetric Kato-Rellich potential $V_{\vec{e}} \in L^{2}(\vec{e})$ we have}

(1) For $\Phi \notin 2 \pi \mathbb{Q}$, the spectrum on $\sigma_{\text {cont }}^{\Phi}$ is purely singular continuous.

(2) For $\Phi \in 2 \pi \mathbb{Q}$, the spectrum on $\sigma_{\text {cont }}^{\Phi}$ is absolutely continuous.

Of course our results only describe the quantum graph model of graphene in a magnetic field, which is both single-electron and high contrast. In particular, we believe that the isolated eigenvalues are unphysical, being an artifact of the graph model which does not allow something similar to actual Coulomb potentials close to the carbon atoms or dissolving of eigenstates supported on edges in the bulk. However, there are reasons to expect that continuous spectrum of the quantum graph operator (thus the Cantor set described in this paper) does adequately capture the experimental properties of graphene in the magnetic field [14]. In particular, certain properties of the density of states of our model (which starts from actual differential operator and is exact in every step) better correspond to the experimental observations [24] than those of the commonly used tight-binding model [4]. We refer the reader to [13,14] for detail. Finally, our analysis provides full description of the spectrum of the tight-binding Hamiltonian as well. Moreover, the applicability of our model is certainly not limited to graphene.

Earlier work showing Cantor spectrum on quantum graphs with magnetic fields, e.g. for the square lattice [11] and magnetic chains studied in [19], has been mostly limited to applications of the Cantor spectrum of the almost Mathieu operator [5,45]. On the honeycomb graph, we can no longer resort to this operator. The discrete operator is then matrix-valued and can be further reduced to a one-dimensional discrete quasiperiodic operator using supersymmetry. The resulting discrete operator is a singular Jacobi matrix ${ }^{3}$ Cantor spectrum (in fact, a stronger, dry ten martini type statement) for Jacobi matrices of this type has been studied in the framework of the extended Harper's model [29]. However, the method of [29] that goes back to that of [6] relies on (almost) reducibility, and thus in particular is not applicable in absence of (dual) absolutely continuous spectrum which is prevented by singularity. Similarly, the method of [5] breaks down in presence of singularity in the Jacobi matrix as well. Instead, we present a novel way that exploits singularity rather than circumvents it by showing that the singularity leads to vanishing of the measure of the spectrum, and thus Cantor structure and singular continuity,

\footnotetext{
3 A Jacobi matrix is called singular if its off-diagonal entries are not bounded away from zero.
} 
once (4) of Theorem 1 is established. ${ }^{4}$ Our method applies also to proving zero measure Cantor spectrum of the extended Harper's model whenever the corresponding Jacobi matrix is singular and either the Lyapunov exponent is zero on the spectrum or one can estimate the measure of the spectrum for the rational frequency. The latter is also useful for estimating the Hausdorff dimension and was only available previously for the almost Mathieu operator $[9,42]$ with, in particular, the method of [9] extendable only to situations when measure of the spectrum is not zero, and the method of [42] very almost Mathieu specific. Here we develop a novel method, that applies to general singular Jacobi matrices (see e.g. Lemma 6.8) for which one can establish a Chambers-type formula.

As mentioned, our first step is a reduction to a matrix-valued tight-binding hexagonal model. This leads to an operator $Q_{\Lambda}$ defined in (4.1). This operator has been studied before for the case of rational magnetic flux (see [28] and references therein). Our analysis gives complete spectral description for this operator as well.

Theorem 3 The spectrum of $Q_{\Lambda}(\Phi)$ is

- a finite union of intervals and purely absolutely continuous for $\Phi / 2 \pi=$ $p / q$, which is a reduced rational number, with the following measure estimate

$$
\left|\sigma\left(Q_{\Lambda}(\Phi)\right)\right| \leq \frac{C}{\sqrt{q}}
$$

where $C>0$ is an absolute constant.

- singular continuous and a zero measure Cantor set for $\Phi \notin 2 \pi \mathbb{Q}$,

- a set of Hausdorff dimension $\operatorname{dim}_{H}\left(\sigma\left(Q_{\Lambda}(\Phi)\right)\right) \leq 1 / 2$ for generic ${ }^{5} \Phi$.

Remark 1 We will show that the constant $C$ in the first item can be bounded by $\frac{8 \sqrt{6 \pi}}{9}$.

The theory of magnetic Schrödinger operators on graphs can be found in [41]. The effective one-particle graph model for graphene without magnetic fields was introduced in [39]. After incorporating a magnetic field according to [41] in the model of [39], the reduction of differential operators on the graph to a discrete tight-binding operator can be done using Krein's extension theory for general self-adjoint operators on Hilbert spaces. This technique has been introduced in [46] for magnetic quantum graphs on the square lattice. The

\footnotetext{
${ }^{4}$ We note that singular continuity of the spectrum of critical extended Harper's model (including for parameters leading to singularity in the corresponding Jacobi matrix) has been proved recently in $[7,30]$ without establishing the Cantor nature.

5 See Footnote 2.
} 
quantum graph nature of the differential operators causes, besides the contribution of the tight-binding operator to the continuous spectrum, a contribution to the point spectrum that consists of Dirichlet eigenfunctions vanishing at every vertex.

In this paper we develop the corresponding reduction for the hexagonal structure and derive spectral conclusions in a way that allows easy generalization to other planar graphs spanned by two basis vectors. In particular, our techniques should be applicable to study quantum graphs on the triangular lattice, which will be pursued elsewhere.

One of the striking properties of graphene is the presence of a linear dispersion relation which leads to the formation of conical structures of the dispersion surfaces in the Brillouin zone, see Fig. 5. The points where the cones match are called Dirac points to account for the special dispersion relation. We use a spectral equivalence between the magnetic Schrödinger operators on the graph and tight-binding operators that is based on Krein's theory in a version introduced in $[47,48]$. In particular, the bands of the graph model always touch at the Dirac points and are shown to have open gaps at the band edges of the associated Hill operator if the magnetic flux is non-trivial. We obtain the preceding results by first proving a bound on the operator norm of the tight-binding operator and analytic perturbation theory.

In [39] it was shown that the Dirichlet contribution to the spectrum in the non-magnetic case is generated by compactly supported eigenfunctions and that this is the only contribution to the point spectrum of the Schrödinger operator on the graph. We extend this result to magnetic Schrödinger operators on hexagonal graphs. Let $H_{p p}$ be the pure point subspace accociated with $H^{B}$. Then

Theorem 4 For any $\Phi, H_{p p}$ is spanned by compactly supported eigenfunctions (in fact, by double hexagonal states).

While for the rational $\Phi$ the proof is based on ideas similar to those of [39], for the irrational $\Phi$ we no longer have an underlying periodicity thus cannot use the arguments of [36]. After showing that there are double hexagonal state eigenfunctions for each Dirichlet eigenvalue, it remains to show their completeness. While there are various ways to show that all $\ell^{1}$ (in a suitable sense) eigenfunctions are in the closure of the span of double hexagonal states, the $\ell^{2}$ condition is more elusive. Bridging the gap between $\ell^{1}$ and $\ell^{2}$ has been a known difficult problem in several other scenarios $[1,7,10,32]$. Here we achieve this by constructing, for each $\Phi$, an operator that would have all slowly decaying $\ell^{2}$ eigenfunctions in its kernel and showing its invertibility. This is done using constructive arguments and properties of holomorphic families of operators. We note that, to the best of our knowledge, Theorem 4 is the first 
result of this sort in absence of periodicity, and our way of bridging the gap between $\ell^{1}$ and $\ell^{2}$ is also a novel argument.

\subsection{Outline}

Section 2 serves as background, in particular it reviews results on the honeycomb quantum graph model without magnetic fields. In Sect. 3, we introduce the magnetic Schrödinger operator $H^{B}$ show that this one is unitarily equivalent to a non-magnetic Schrödinger operator $\Lambda^{B}$ with magnetic contributions moved into the boundary conditions. In Sect. 4 , we present several key ingredients of the proofs of the main theorems: Lemmas 4.1-4.4. Lemma 4.1 involves a further reduction from $\Lambda^{B}$ to a two-dimensional tight-binding Hamiltonian $Q_{\Lambda}(\Phi)$, and Lemmas 4.2-4.4 reveal the topological structure of $\sigma\left(Q_{\Lambda}(\Phi)\right)$ (thus proving the topological part of Theorem 3). The proofs of Lemmas 4.1-4.4 are given is Sects. 5, 6 and 7. Section 8 is devoted to a complete spectral analysis of $H^{B}$, thus proving Theorem 1, with the analysis of Dirichlet spectrum in Sect. 8.2, where, in particular, we prove Theorem 4; absolutely continuous spectrum for rational flux in Sect. 8.3, singular continuous spectrum for irrational flux in Sect. 8.4 (thus proving Theorem 2). Since most of the proofs for different parts of Theorems 1-4 are distributed throughout the paper, we give an index to them, for the reader's convenience in Sect. 8.5.

\section{Preliminaries}

\subsection{Notation}

Given a graph $G$, we denote the set of edges of $G$ by $\mathcal{E}(G)$, the set of vertices by $\mathcal{V}(G)$, and the set of edges adjacent to a vertex $\boldsymbol{v} \in \mathcal{V}(G)$ by $\mathcal{E}_{\boldsymbol{v}}(G)$.

For an operator $H$, let $\sigma(H)$ be its spectrum and $\rho(H)$ be the resolvent set.

The space $c_{00}$ is the space of all infinite sequences with only finitely many non-zero terms (finitely supported sequences). We denote by $\Omega^{i}\left(\mathbb{R}^{2}\right)$ the vector space of all $i$-covectors or differential forms of degree $i$ on $\mathbb{R}^{2}$.

For a set $U \subseteq \mathbb{R}$, let $|U|$ be its Lebesgue measure. We define $\mathbb{T}_{2}^{*}:=$ $\mathbb{R}^{2} /(2 \pi \mathbb{Z})^{2}$ and $\mathbb{T}:=\mathbb{T}_{1}:=\mathbb{R} / \mathbb{Z}$.

\section{List of main symbols used in this article.}

- $\boldsymbol{r}_{0}$ and $\boldsymbol{r}_{1}$ are the vertices of the fundamental cell (2.1).

- $\vec{f}, \vec{g}, \vec{h}$ are the vectors of the fundamental cell (2.2).

- $W_{\Lambda}:=\left\{\vec{f}, \vec{g}, \vec{h}, \boldsymbol{r}_{0}, \boldsymbol{r}_{1}\right\}$ is the fundamental cell.

- $\vec{b}_{1}, \vec{b}_{2}$ are the basis vectors of the lattice (2.3).

- $\Lambda$ is the metric honeycomb graph (2.4). 
- $[v],[\vec{e}]$ denotes the translate of a vertex $v$ or edge $\vec{e}$ into the fundamental cell (2.5).

- $v=\left(\gamma_{1}, \gamma_{2},[\boldsymbol{v}]\right), \vec{e}=\left(\gamma_{1}, \gamma_{2},[\vec{e}]\right)$ are defined in the paragraph below (2.5).

- $i, t$ map edges to their respective initial and terminal vertex (2.6).

- $\kappa_{\vec{e}}$ is the chart defined in (2.9).

- $\mathcal{H}^{n}$ are the Sobolev spaces (2.10).

- $H_{\vec{e}}$ is the maximal Schrödinger operator on an edge $\vec{e}$ (2.11).

- $V$ is the potential as defined in (2.12).

- $H^{D}$ is the Schrödinger operator with Dirichlet boundary conditions (2.14).

- $H$ is the Schrödinger operator without magnetic field (2.16).

- $T_{\gamma}^{\text {st }}$ are lattice translations (2.17).

- $H(\boldsymbol{k})$ are non-magnetic Schrödinger operators satisfying Floquet boundary conditions (2.21).

- $\psi_{\lambda, 1}, \psi_{\lambda, 2}$ are solutions to the boundary value problem stated in (2.23).

- $\eta(\lambda)$ is introduced below (2.25).

- $c_{\lambda, \vec{e}}$ and $s_{\lambda, \vec{e}}$ are defined in (2.27).

- Hill potential $V_{\text {Hill }}$ and Hill operator $H_{\text {Hill }}$ are defined in (2.33) and (2.34).

- $\Delta(\lambda)$ is the Floquet discriminant defined in (2.37).

- $H_{\Phi, \theta} \in \mathcal{L}\left(l^{2}(\mathbb{Z})\right)$ is the Jacobi operator defined in (2.41), with spectrum $\Sigma_{\Phi, \theta}$ and $\Sigma_{\Phi}:=\bigcup_{\theta \in \mathbb{T}_{1}} \Sigma_{\Phi, \theta}$.

- $\Theta$ is the set of zeros of $c(\theta)$ as defined in Sect. 2.3.1.

- $A^{\lambda}, A_{n}^{\lambda}, \widetilde{A}^{\lambda}$ and are the transfer, $n$-step transfer, and normalized transfer matrix defined in (2.42), (2.43), and (2.46).

- $D^{\lambda}$ and $D_{n}^{\lambda}$ are derived from transfer matrices in (2.49).

- $L(\lambda, \Phi)$ is the Lyapunov exponent defined in (2.44).

- Vector potential $A$, integrated vector potential $\beta$, and flux $\Phi$ are defined in (3.1) and (3.2).

- $H^{B}$ is the Schrödinger operator introduced in (3.7)

- $\Lambda^{B}$ is the Schrödinger operator introduced in (3.14).

- $Q_{\Lambda}(\Phi)$ is the tight-binding operator stated in (4.1).

- $\tau_{0}$ and $\tau_{1}$ are discrete magnetic translation operators defined in (4.2).

- $\gamma(\lambda)$ is defined in (7.8), $M(\lambda, \Phi)$ is given in (7.9), $K_{\lambda}$ is defined in (7.10).

- $T_{\gamma}^{B}$ are magnetic translation defined in (8.1).

\subsection{Hexagonal quantum graphs}

This subsection is devoted to reviewing hexagonal quantum graphs without magnetic fields. The readers could refer to [39] for details. We include some material here that serves as a preparation for the study of quantum graphs with magnetic fields in Sect. 3. 
A model for effective one electron behavior in graphene is given by a hexagonal graph with Schrödinger operators defined on each edge [39]. The hexagonal graph $\Lambda$ is obtained by translating its fundamental cell $W_{\Lambda}$, the red colored part of Fig. 1, consisting of vertices

$$
\boldsymbol{r}_{0}:=(0,0) \text { and } \boldsymbol{r}_{1}:=\left(\frac{1}{2}, \frac{\sqrt{3}}{2}\right)
$$

and edges

$$
\begin{aligned}
\vec{f} & :=\operatorname{conv}\left(\left\{\boldsymbol{r}_{0}, \boldsymbol{r}_{1}\right\}\right) \backslash\left\{\boldsymbol{r}_{0}, \boldsymbol{r}_{1}\right\}, \\
\vec{g} & :=\operatorname{conv}\left(\left\{\boldsymbol{r}_{0},(-1,0)\right\}\right) \backslash\left\{\boldsymbol{r}_{0},(-1,0)\right\}, \text { and } \\
\vec{h} & :=\operatorname{conv}\left(\left\{\boldsymbol{r}_{0},\left(\frac{1}{2},-\frac{\sqrt{3}}{2}\right)\right\}\right) \backslash\left\{\boldsymbol{r}_{0},\left(\frac{1}{2},-\frac{\sqrt{3}}{2}\right)\right\},
\end{aligned}
$$

along the basis vectors of the lattice. The basis vectors are

$$
\vec{b}_{1}:=\left(\frac{3}{2}, \frac{\sqrt{3}}{2}\right) \text { and } \vec{b}_{2}:=(0, \sqrt{3})
$$

and so the hexagonal graph $\Lambda \subset \mathbb{R}^{2}$ is given by the range of a $\mathbb{Z}^{2}$-action on the fundamental domain $W_{\Lambda}$

$$
\Lambda:=\left\{\boldsymbol{x} \in \mathbb{R}^{2}: x=\gamma_{1} \vec{b}_{1}+\gamma_{2} \vec{b}_{2}+\boldsymbol{y} \text { for } \boldsymbol{\gamma} \in \mathbb{Z}^{2} \text { and } \boldsymbol{y} \in W_{\Lambda}\right\} .
$$

The fundamental domain of the dual lattice can be identified with the dual 2-torus $\mathbb{T}_{2}^{*}$.

For any vertex $\boldsymbol{v} \in \mathcal{V}(\Lambda)$, we denote by $[\boldsymbol{v}] \in \mathcal{V}\left(W_{\Lambda}\right)$ the unique vertex, $\boldsymbol{r}_{0}$ or $\boldsymbol{r}_{1}$, for which there is $\boldsymbol{\gamma} \in \mathbb{Z}^{2}$ such that

$$
\boldsymbol{v}=\gamma_{1} \vec{b}_{1}+\gamma_{2} \vec{b}_{2}+[\boldsymbol{v}] .
$$

We will occasionally denote $\boldsymbol{v}$ by $\left(\gamma_{1}, \gamma_{2},[\boldsymbol{v}]\right)$ to emphasize the location of $\boldsymbol{v}$. We also introduce a similar notation for edges. For an edge $\vec{e} \in \mathcal{E}(\Lambda)$, we will sometimes denote it by $\left(\gamma_{1}, \gamma_{2},[\vec{e}]\right)$. Finally, for any $\boldsymbol{x} \in \Lambda$, we will also denote its unique preimage in $W_{\Lambda}$ by $[x]{ }^{6}$

We can orient the edges in terms of initial and terminal maps

$$
i: \mathcal{E}(\Lambda) \rightarrow \mathcal{V}(\Lambda) \text { and } t: \mathcal{E}(\Lambda) \rightarrow \mathcal{V}(\Lambda)
$$

6 So that $y$ in $(2.4)=[x]$. 


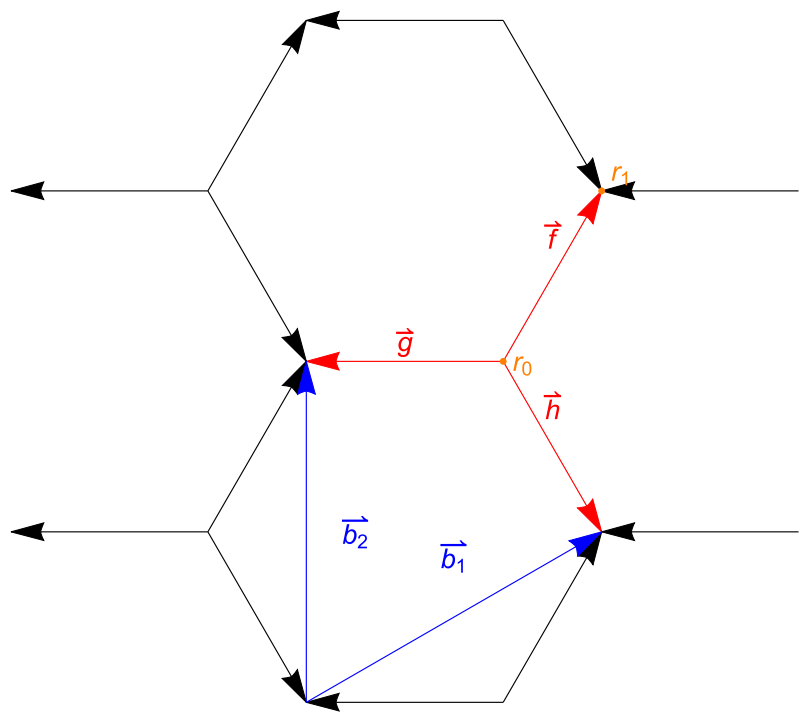

Fig. 1 The fundamental cell $W_{\Lambda}$, colored in red and including points $r_{0}, r_{1}$, and lattice basis vectors of $\Lambda$ (color figure online)

where $i$ and $t$ map edges to their initial and terminal ends respectively. It suffices to specify the orientation on the edges of the fundamental domain $W_{\Lambda}$ to obtain an oriented graph $\Lambda$

$$
\begin{aligned}
& i(\vec{f})=i(\vec{g})=i(\vec{h})=\boldsymbol{r}_{0}, \\
& t(\vec{f})=\boldsymbol{r}_{1}, t(\vec{g})=\boldsymbol{r}_{1}-\vec{b}_{1}, \text { and } t(\vec{h})=\boldsymbol{r}_{1}-\vec{b}_{2} .
\end{aligned}
$$

For arbitrary $\vec{e} \in \mathcal{E}(\Lambda)$, we then just extend those maps by

$$
i(\vec{e}):=\gamma_{1} \vec{b}_{1}+\gamma_{2} \vec{b}_{2}+i([\vec{e}]) \text { and } t(\vec{e}):=\gamma_{1} \vec{b}_{1}+\gamma_{2} \vec{b}_{2}+t([\vec{e}]) .
$$

Let $i(\Lambda)=\{\boldsymbol{v} \in \mathcal{V}(\Lambda): \boldsymbol{v}=i(\vec{e})$ for some $\vec{e} \in \mathcal{E}(\Lambda)\}$ be the collection of initial vertices, and $t(\Lambda)=\{\boldsymbol{v} \in \mathcal{V}(\Lambda): \boldsymbol{v}=t(\vec{e})$ for some $\vec{e} \in \mathcal{E}(\Lambda)\}$ be the collection of terminal ones. It should be noted that based on our orientation, $\mathcal{V}(\Lambda)$ is a disjoint union of $i(\Lambda)$ and $t(\Lambda)$.

Every edge $\vec{e} \in \mathcal{E}(\Lambda)$ is of length one and thus has a canonical chart

$$
\begin{aligned}
\kappa_{\vec{e}}: & \vec{e} \rightarrow(0,1), \\
& (i(\vec{e}) x+t(\vec{e})(1-x)) \mapsto x
\end{aligned}
$$

that allows us to define function spaces and operators on $\vec{e}$ and finally on the entire graph. For $n \in \mathbb{N}_{0}$, the Sobolev space $\mathcal{H}^{n}(\mathcal{E}(\Lambda))$ on $\Lambda$ is the Hilbert space direct sum 
Fig. 2 The potential is the same on all edges and symmetric with respect to the centre of the edge

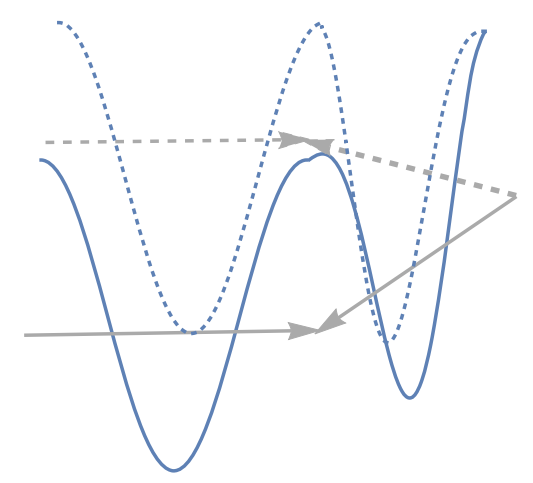

$$
\mathcal{H}^{n}(\mathcal{E}(\Lambda)):=\bigoplus_{\vec{e} \in \mathcal{E}(\Lambda)} \mathcal{H}^{n}(\vec{e})
$$

On every edge $\vec{e} \in \mathcal{E}(\Lambda)$ we define the maximal Schrödinger operator

$$
\begin{aligned}
& H_{\vec{e}}: \mathcal{H}^{2}(\vec{e}) \subset L^{2}(\vec{e}) \rightarrow L^{2}(\vec{e}) \\
& H_{\vec{e}} \psi_{\vec{e}}:=-\psi_{\vec{e}}^{\prime \prime}+V_{\vec{e}} \psi_{\vec{e}}
\end{aligned}
$$

with Kato-Rellich potential $V_{\vec{e}} \in L^{2}(\vec{e})$ that is the same on every edge and even with respect to the center of the edge, see Fig. 2. Let

$$
V(t)=V_{\vec{e}}\left(\left(\kappa_{\vec{e}}\right)^{-1}(t)\right)
$$

Then

$$
V(t)=V(1-t)
$$

One self-adjoint restriction of (2.11) is the Dirichlet operator

$$
\begin{aligned}
& H^{D}:=\bigoplus_{\vec{e} \in \mathcal{E}(\Lambda)}\left(\mathcal{H}_{0}^{1}(\vec{e}) \cap \mathcal{H}^{2}(\vec{e})\right) \subset L^{2}(\mathcal{E}(\Lambda)) \rightarrow L^{2}(\mathcal{E}(\Lambda)) \\
& \left(H^{D} \psi\right)_{\vec{e}}:=H_{\vec{e}} \psi_{\vec{e}},
\end{aligned}
$$

where $\mathcal{H}_{0}^{1}(\vec{e})$ is the closure of compactly supported smooth functions in $\mathcal{H}^{1}(\vec{e})$. The Hamiltonian we will use to model the graphene without magnetic fields is the self-adjoint [36] operator $H$ on $\Lambda$ with Neuman type boundary conditions 


$$
\begin{array}{r}
D(H):=\left\{\psi=\left(\psi_{\vec{e}}\right) \in \mathcal{H}^{2}(\mathcal{E}(\Lambda)): \text { for all } \boldsymbol{v} \in \mathcal{V}(\Lambda), \psi_{\vec{e}_{1}}(\boldsymbol{v})\right. \\
=\psi_{\vec{e}_{2}}(\boldsymbol{v}) \text { if } \vec{e}_{1}, \vec{e}_{2} \in \mathcal{E}_{\boldsymbol{v}}(\Lambda) \\
\text { and } \left.\sum_{\vec{e} \in \mathcal{E}_{\boldsymbol{v}}(\Lambda)} \psi_{\vec{e}}^{\prime}(\boldsymbol{v})=0\right\}
\end{array}
$$

and defined by

$$
\begin{aligned}
& H: D(H) \subset L^{2}(\mathcal{E}(\Lambda)) \rightarrow L^{2}(\mathcal{E}(\Lambda)) \\
& (H \psi)_{\vec{e}}:=H_{\vec{e}} \psi_{\vec{e}} .
\end{aligned}
$$

Remark 2 The self-adjointness of $H$ will also follow from the self-adjointness of the more general family of magnetic Schrödinger operators that is obtained in Sec. 7.

Remark 3 The orientation is chosen so that all edges at any vertex are either all incoming or outgoing. Thus, there is no need to distinguish those situations in terms of a directional derivative in the boundary conditions (2.15).

\subsubsection{Floquet-Bloch decomposition}

Operator $H$ commutes with the standard lattice translations

$$
\begin{aligned}
T_{\gamma}^{\mathrm{st}}: & L^{2}(\mathcal{E}(\Lambda)) \rightarrow L^{2}(\mathcal{E}(\Lambda)) \\
f & \mapsto f\left(\cdot-\gamma_{1} \vec{b}_{1}-\gamma_{2} \vec{b}_{2}\right)
\end{aligned}
$$

for any $\gamma \in \mathbb{Z}^{2}$. In terms of those, we define the Floquet-Bloch transform for $\boldsymbol{x} \in \mathcal{E}\left(W_{\Lambda}\right)$ and $\boldsymbol{k} \in \mathbb{T}_{2}^{*}$ first on function $f \in C_{c}(\mathcal{E}(\Lambda))$

$$
(U f)(\boldsymbol{k}, \boldsymbol{x}):=\sum_{\boldsymbol{\gamma} \in \mathbb{Z}^{2}}\left(T_{\boldsymbol{\gamma}}^{\mathrm{st}} f\right)(\boldsymbol{x}) e^{i\langle\boldsymbol{k}, \boldsymbol{\gamma}\rangle}
$$

and then extend it to a unitary map $U \in \mathcal{L}\left(L^{2}(\mathcal{E}(\Lambda)), L^{2}\left(\mathbb{T}_{2}^{*} \times \mathcal{E}\left(W_{\Lambda}\right)\right)\right)$ with inverse

$$
\left(U^{-1} \varphi\right)(\boldsymbol{x})=\int_{\mathbb{T}_{2}^{*}} \varphi(\boldsymbol{k},[\boldsymbol{x}]) e^{-i\langle\boldsymbol{\gamma}, \boldsymbol{k}\rangle} \frac{d \boldsymbol{k}}{(2 \pi)^{2}},
$$

where $[\boldsymbol{x}] \in \mathcal{E}\left(W_{\Lambda}\right)$ is the unique pre-image of $\boldsymbol{x}$ in $W_{\Lambda}$, and $\boldsymbol{\gamma} \in \mathbb{Z}^{2}$ is defined by $\boldsymbol{x}=\gamma_{1} \vec{b}_{1}+\gamma_{2} \vec{b}_{2}+[\boldsymbol{x}]$.

Then standard Floquet-Bloch theory implies that there is a direct integral representation of $H$

$$
U H U^{-1}=\int_{\mathbb{T}_{2}^{*}}^{\oplus} H(\boldsymbol{k}) \frac{d \boldsymbol{k}}{(2 \pi)^{2}}
$$



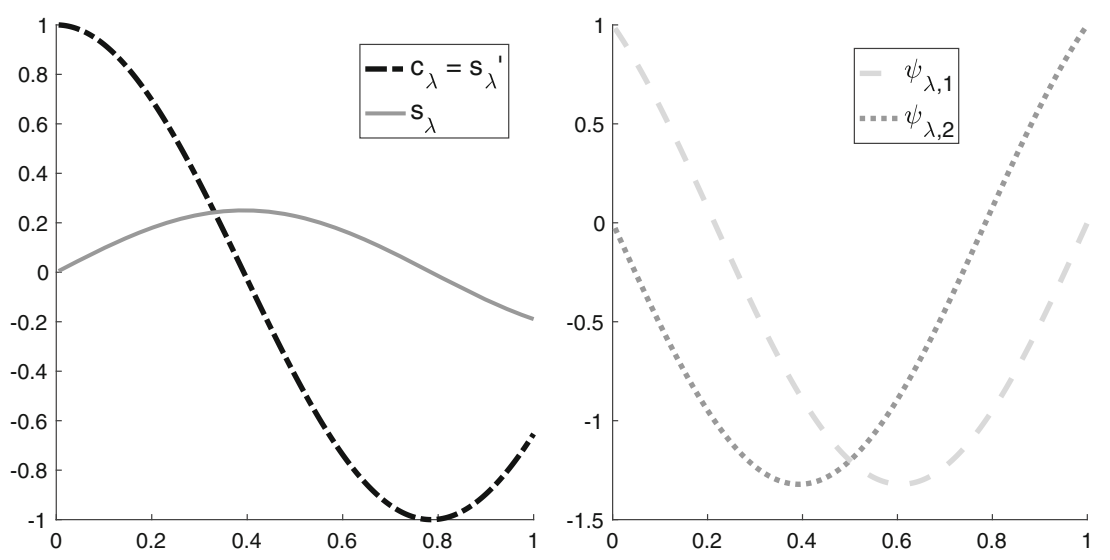

Fig. 3 For zero potential, we illustrate functions (2.23) and (2.27) for $\lambda=16$

in terms of self-adjoint operators $H(\boldsymbol{k})$

$$
\begin{aligned}
& H(\boldsymbol{k}): D(H(\boldsymbol{k})) \subset L^{2}\left(\mathcal{E}\left(W_{\Lambda}\right)\right) \rightarrow L^{2}\left(\mathcal{E}\left(W_{\Lambda}\right)\right) \\
& (H(\boldsymbol{k}) \psi)_{\vec{e}}:=\left(H_{\vec{e}} \psi_{\vec{e}}\right)
\end{aligned}
$$

on the fundamental domain $W_{\Lambda}$ with Floquet boundary conditions

$$
\begin{aligned}
& D(H(\boldsymbol{k})):=\left\{\psi \in \mathcal{H}^{2}\left(\mathcal{E}\left(W_{\Lambda}\right)\right): \psi_{\vec{f}}\left(\boldsymbol{r}_{0}\right)=\psi_{\vec{g}}\left(\boldsymbol{r}_{0}\right)=\psi_{\vec{h}}\left(\boldsymbol{r}_{0}\right)\right. \text { and } \\
& \sum_{\vec{e} \in \mathcal{E}_{r_{0}}(\Lambda)} \psi_{\vec{e}}^{\prime}\left(r_{0}\right)=0 \\
& \text { as well as } \psi_{\vec{f}}\left(\boldsymbol{r}_{1}\right)=e^{i k_{1}} \psi_{\vec{g}}\left(\boldsymbol{r}_{1}-\vec{b}_{1}\right)=e^{i k_{2}} \psi_{\vec{h}}\left(\boldsymbol{r}_{1}-\vec{b}_{2}\right) \\
& \text { and } \left.\psi_{\vec{f}}^{\prime}\left(\boldsymbol{r}_{1}\right)+e^{i k_{1}} \psi_{\vec{g}}^{\prime}\left(\boldsymbol{r}_{1}-\vec{b}_{1}\right)+e^{i k_{2}} \psi_{\vec{h}}^{\prime}\left(\boldsymbol{r}_{1}-\vec{b}_{2}\right)=0\right\} \text {. }
\end{aligned}
$$

Fix an edge $\vec{e} \in \mathcal{E}(\Lambda)$ and $\lambda \notin \sigma\left(H^{D}\right)$. There are linearly independent $\mathcal{H}^{2}(\vec{e})$-solutions $\psi_{\lambda, 1, \vec{e}}$ and $\psi_{\lambda, 2, \vec{e}}$ to the equation $H_{\vec{e}} \psi_{\vec{e}}=\lambda \psi_{\vec{e}}$ with the following boundary condition (Fig. 3)

$$
\begin{aligned}
& \psi_{\lambda, 1, \vec{e}}(i(\vec{e}))=1, \quad \psi_{\lambda, 1, \vec{e}}(t(\vec{e}))=0, \\
& \psi_{\lambda, 2, \vec{e}}(i(\vec{e}))=0, \quad \text { and } \psi_{\lambda, 2, \vec{e}}(t(\vec{e}))=1
\end{aligned}
$$

Any eigenfunction to operators $H(\boldsymbol{k})$, with eigenvalues away from $\sigma\left(H^{D}\right)$, can therefore be written in terms of thosefunctions for constants $a, b \in \mathbb{C}$ 


$$
\psi:= \begin{cases}a \psi_{\lambda, 1, \vec{f}}+b \psi_{\lambda, 2, \vec{f}} & \text { along edge } \vec{f} \\ a \psi_{\lambda, 1, \vec{g}}+e^{-i k_{1}} b \psi_{\lambda, 2, \vec{g}} & \text { along edge } \vec{g} \\ a \psi_{\lambda, 1, \vec{h}}+e^{-i k_{2}} b \psi_{\lambda, 2, \vec{h}} & \text { along edge } \vec{h}\end{cases}
$$

with the continuity conditions of (2.22) being already incorporated in the representation of $\psi$. Imposing the conditions stated on the derivatives in (2.22) shows that $\psi$ is non-trivial ( $a, b$ not both equal to zero) and therefore an eigenfunction with eigenvalue $\lambda \in \mathbb{R}$ to $H(\boldsymbol{k})$ iff

$$
\eta(\lambda)^{2}=\frac{\left|1+e^{i k_{1}}+e^{i k_{2}}\right|^{2}}{9}
$$

with $\eta(\lambda):=\frac{\psi_{\lambda, 2, \vec{e}}^{\prime}(t(\vec{e}))}{\psi_{\lambda, 2, \vec{e}}^{\prime}(i(\vec{e}))}$ well-defined away from the Dirichlet spectrum.

By noticing that the range of the function on the right-hand side of (2.25) is $[0,1]$, the following spectral characterization is obtained [39, Theorem 3.6].

Theorem 5 As a set, the spectrum of $H$ away from the Dirichlet spectrum is given by

$$
\sigma(H) \backslash \sigma\left(H^{D}\right)=\{\lambda \in \mathbb{R}:|\eta(\lambda)| \leq 1\} \backslash \sigma\left(H^{D}\right) .
$$

\subsubsection{Dirichlet-to-Neuman map}

Fix an edge $\vec{e} \in \mathcal{E}(\Lambda)$. Let $c_{\lambda, \vec{e}}, s_{\lambda, \vec{e}}$, which for $V_{\vec{e}}=0$ reduce to just $c_{\lambda, \vec{e}}=$ $\cos (\sqrt{\lambda} \bullet)$ and $s_{\lambda, \vec{e}}=\sin (\sqrt{\lambda} \bullet) / \sqrt{\lambda}$, be solutions to $H_{\vec{e}} \psi_{\vec{e}}=\lambda \psi_{\vec{e}}$ with the following boundary condition

$$
\left(\begin{array}{l}
c_{\lambda, \vec{e}}(i(\vec{e})) s_{\lambda, \vec{e}}(i(\vec{e})) \\
c_{\lambda, \vec{e}}^{\prime}(i(\vec{e})) s_{\lambda, \vec{e}}^{\prime}(i(\vec{e}))
\end{array}\right)=\left(\begin{array}{ll}
1 & 0 \\
0 & 1
\end{array}\right)
$$

We point out that $c_{\lambda}(t):=c_{\lambda, \vec{e}}\left(\kappa_{\vec{e}}^{-1}(t)\right)$ and $s_{\lambda}(t):=s_{\lambda, \vec{e}}\left(\kappa_{\vec{e}}^{-1}(t)\right)$ are independent of $\vec{e}$. They are clearly solutions to $-\psi^{\prime \prime}+V \psi=\lambda \psi$ on $(0,1)$, with $c_{\lambda}(0)=1, c_{\lambda}^{\prime}(0)=0, s_{\lambda}(0)=0, s_{\lambda}^{\prime}(0)=1$, where $V$ is defined in (2.12).

Then for $\lambda \notin \sigma\left(H^{D}\right)$, namely when $s_{\lambda}(1) \neq 0$, any $\mathcal{H}^{2}(\vec{e})$-solution $\psi_{\lambda, \vec{e}}$ can be written as a linear combination of $c_{\lambda, \vec{e}}, s_{\lambda, \vec{e}}$

$$
\psi_{\lambda, \vec{e}}(\boldsymbol{x})=\frac{\psi_{\lambda, \vec{e}}(t(\vec{e}))-\psi_{\lambda, \vec{e}}(i(\vec{e})) c_{\lambda}(1)}{s_{\lambda}(1)} s_{\lambda, \vec{e}}(\boldsymbol{x})+\psi_{\lambda, \vec{e}}(i(\vec{e})) c_{\lambda, \vec{e}}(\boldsymbol{x})
$$


The Dirichlet-to-Neuman map is defined by

$$
m(\lambda):=\frac{1}{s_{\lambda}(1)}\left(\begin{array}{cc}
-c_{\lambda}(1) & 1 \\
1 & -s_{\lambda}^{\prime}(1)
\end{array}\right)
$$

with the property that for $\psi_{\lambda, \vec{e}}$ as in (2.28), one has

$$
\left(\begin{array}{c}
\psi_{\lambda, \vec{e}}^{\prime}(i(\vec{e})) \\
-\psi_{\lambda, \vec{e}}^{\prime}(t(\vec{e}))
\end{array}\right)=m(\lambda)\left(\begin{array}{c}
\psi_{\lambda, \vec{e}}(i(\vec{e})) \\
\psi_{\lambda, \vec{e}}(t(\vec{e}))
\end{array}\right)
$$

For the second component, the constancy of the Wronskian is used. Since $V(t)$ is assumed to be even, the intuitive relation

$$
c_{\lambda}(1)=s_{\lambda}^{\prime}(1)
$$

remains also true for non-zero potentials.

For $\lambda \notin \sigma\left(H^{D}\right)$, by expressing $c_{\lambda}(1)$ in terms of $\psi_{\lambda, 1, \vec{e}}$ and $\psi_{\lambda, 2, \vec{e}}$, it follows immediately that

$$
\eta(\lambda)=s_{\lambda}^{\prime}(1)
$$

\subsubsection{Relation to Hill operators}

Using the potential $V(t)$ (2.12), we define the $\mathbb{Z}$-periodic Hill potential $V_{\mathrm{Hill}} \in$ $L_{\text {loc }}^{2}(\mathbb{R})$.

$$
V_{\text {Hill }}(t):=V(t(\bmod 1)),
$$

for $t \in \mathbb{R}$. The associated self-adjoint Hill operator on the real line is given by

$$
\begin{aligned}
& H_{\text {Hill }}: \mathcal{H}^{2}(\mathbb{R}) \subset L^{2}(\mathbb{R}) \rightarrow L^{2}(\mathbb{R}) \\
& H_{\text {Hill }} \psi:=-\psi^{\prime \prime}+V_{\text {Hill }} \psi .
\end{aligned}
$$

Then $c_{\lambda}, s_{\lambda} \in \mathcal{H}^{2}(0,1)$, extending naturally to $\mathcal{H}_{\text {loc }}^{2}(\mathbb{R})$, become solutions to

$$
H_{\text {Hill }} \psi=\lambda \psi \text {. }
$$

The monodromy matrix associated with $H_{\text {Hill }}$ is the matrix valued function

$$
Q(\lambda):=\left(\begin{array}{ll}
c_{\lambda}(1) & s_{\lambda}(1) \\
c_{\lambda}^{\prime}(1) & s_{\lambda}^{\prime}(1)
\end{array}\right)
$$

and depends by standard ODE theory holomorphically on $\lambda$. Its normalized trace

$$
\Delta(\lambda):=\frac{\operatorname{tr}(Q(\lambda))}{2}=s_{\lambda}^{\prime}(1)
$$




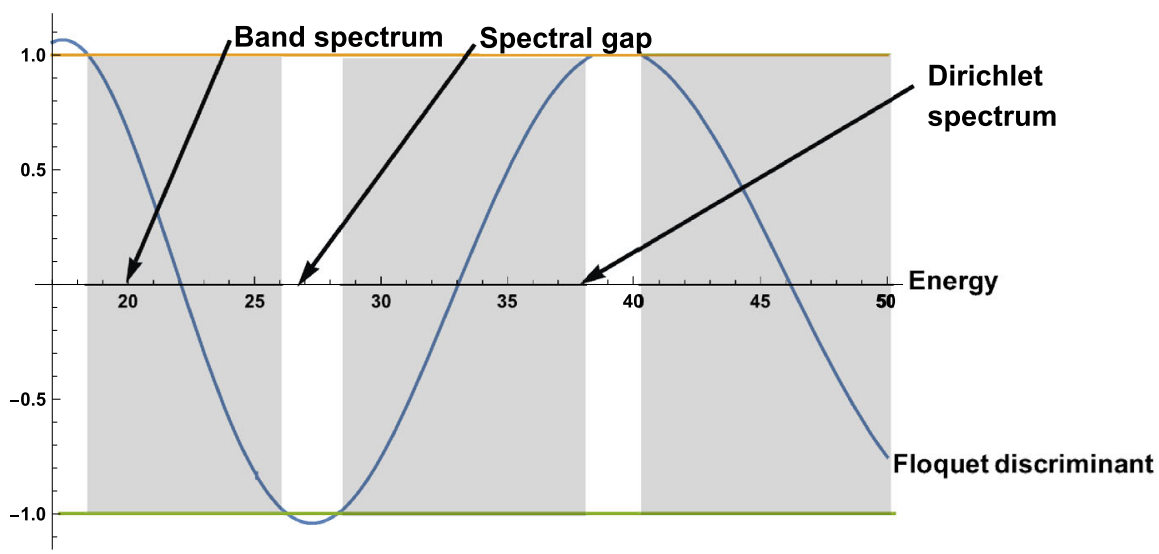

Fig. 4 The Floquet discriminant for a Mathieu potential $V(t)=4 \cos (6 t)$. Energies in shaded regions are inside the band spectrum. Dirichlet eigenvalues are located at the band edges

is called the Hill (aka Floquet) discriminant. In the simplest case when $V_{\text {Hill }}=$ 0 , the Floquet discriminant is just $\Delta(\lambda)=\cos (\sqrt{\lambda})$ for $\lambda \geq 0$.

By the well-known spectral decomposition of periodic differential operators on the line [49], the spectrum of the Hill operator is purely absolutely continuous and satisfies

$$
\sigma\left(H_{\text {Hill }}\right)=\{\lambda \in \mathbb{R}:|\Delta(\lambda)| \leq 1\}=\bigcup_{n=1}^{\infty}\left[\alpha_{n}, \beta_{n}\right]
$$

where $B_{n}:=\left[\alpha_{n}, \beta_{n}\right]$ denotes the $n$-th Hill band with $\beta_{n} \leq \alpha_{n+1}$. We have $\left.\Delta\right|_{\text {int }\left(B_{n}\right)} ^{\prime}(\lambda) \neq 0$.

Putting (2.32) and (2.37) together, we get the following relation

$$
\Delta(\lambda)=\eta(\lambda), \text { for } \lambda \notin \sigma\left(H^{D}\right),
$$

that connects the Hill spectrum with the spectrum of the graphene Hamiltonian.

Also, if $\lambda \in \sigma\left(H^{D}\right)$, then by the symmetry of the potential, the Dirichlet eigenfunction are either even or odd with respect to $\frac{1}{2}$. Thus, Dirichlet eigenvalues can only be located at the edges of the Hill bands, see Fig. 4. Namely,

$$
\Delta(\lambda)= \pm 1, \text { for } \lambda \in \sigma\left(H^{D}\right) .
$$

\subsubsection{Spectral decomposition}

The singular continuous spectrum of $H$ is empty by the direct integral decomposition (2.20) [26]. Due to Thomas [51] there is the characterization, stated also in [37, Corollary 6.11], of the pure point spectrum of fibered operators: 


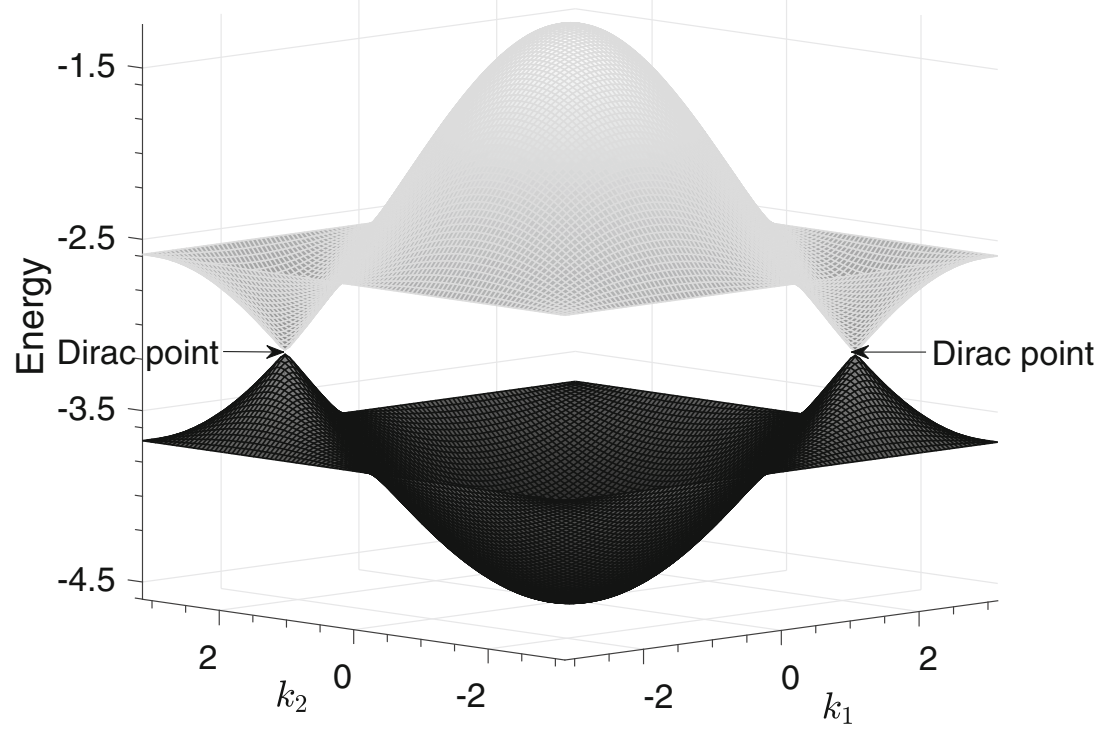

Fig. 5 The first two bands of the Schrödinger operator on the graph with Mathieu potential $V(t)=20 \cos (2 \pi t)$ and no magnetic field showing the characteristic conical Dirac points where the two differently colored bands touch. The two bands are differently colored

$\lambda$ is in the pure point spectrum iff the set $\left\{\boldsymbol{k} \in \mathbb{T}_{2}^{*} ; \lambda_{j}(\boldsymbol{k})=\lambda\right\}$ has positive measure where $\lambda_{j}(\boldsymbol{k})$ is the $j$-th eigenvalue of $H(\boldsymbol{k})$. Away from the Dirichlet spectrum, the condition $\mathbb{R} \ni \lambda=\lambda_{j}(\boldsymbol{k})$ is by (2.25) equivalent to $\Delta(\lambda)^{2}=\frac{\left|1+e^{i k_{1}}+e^{i k_{2}}\right|^{2}}{9}$. Yet, the level-sets of this function are of measure zero. The spectrum of $H$ away from the Dirichlet spectrum is therefore purely absolutely continuous. The Dirichlet spectrum coincides with the point spectrum of $H$ and is spanned by so-called loop states that consist of six Dirichlet eigenfunctions wrapped around each hexagon of the lattice [39, Theorem 3.6(v)]. Hence, the spectral decomposition in the case without magnetic field is given by

Theorem 6 The spectra of $\sigma(H)$ and $\sigma\left(H_{\text {Hill }}\right)$ coincide as sets. Aside from the Dirichlet contribution to the spectrum, $H$ has absolutely continuous spectrum as in Fig. 5 with conical cusps at the points (Dirac points) where two bands on each Hill band meet. The Dirichlet spectrum is contained in the spectrum of $H$, is spanned by loop states supported on single hexagons, and is thus infinitely degenerated. 


\subsection{One-dimensional quasi-periodic Jacobi matrices}

The proof of the main Theorems will involve the study of a one-dimensional quasi-periodic Jacobi matrix. We include several general facts that will be useful.

Let $H_{\Phi, \theta} \in \mathcal{L}\left(l^{2}(\mathbb{Z})\right)$ be a quasi-periodic Jacobi matrix, that is given by

$$
\begin{aligned}
\left(H_{\Phi, \theta} u\right)_{m}= & c\left(\theta+m \frac{\Phi}{2 \pi}\right) u_{m+1}+\overline{c\left(\theta+(m-1) \frac{\Phi}{2 \pi}\right)} u_{m-1} \\
& +v\left(\theta+m \frac{\Phi}{2 \pi}\right) u_{m}
\end{aligned}
$$

Let $\Sigma_{\Phi, \theta}:=\sigma\left(H_{\Phi, \theta}\right)$ be the spectrum of $H_{\Phi, \theta}$ and $\Sigma_{\Phi}=\bigcup_{\theta \in \mathbb{T}_{1}} \Sigma_{\Phi, \theta}$. It is a well known result that for irrational $\frac{\Phi}{2 \pi}$, the set $\Sigma_{\Phi, \theta}$ is independent of $\theta$, thus $\Sigma_{\Phi, \theta}=\Sigma_{\Phi}$. It is also well known that, for any $\Phi, \Sigma_{\Phi}$ has no isolated points. $^{7}$

\subsubsection{Transfer matrix and Lyapunov exponent}

We assume that $c(\theta)$ has finitely many zeros (counting multiplicity), and label them as $\theta_{1}, \theta_{2}, \ldots, \theta_{m} .{ }^{8}$ Let $\Theta:=\cup_{j=1}^{m} \cup_{k \in \mathbb{Z}}\left\{\theta_{j}+k \frac{\Phi}{2 \pi}\right\}$, in particular if $\frac{\Phi}{2 \pi} \in \mathbb{Q}$, then $\Theta$ is a finite set in $\mathbb{T}$.

For $\theta \notin \Theta$, the eigenvalue equation $H_{\Phi, \theta} u=\lambda u$ has the following dynamical reformulation:

$$
\left(\begin{array}{c}
u_{n+1} \\
u_{n}
\end{array}\right)=A^{\lambda}\left(\theta+n \frac{\Phi}{2 \pi}\right)\left(\begin{array}{c}
u_{n} \\
u_{n-1}
\end{array}\right),
$$

where

$$
\mathrm{GL}(2, \mathbb{C}) \ni A^{\lambda}(\theta)=\frac{1}{c(\theta)}\left(\begin{array}{cc}
\lambda-v(\theta) & -\overline{c\left(\theta-\frac{\Phi}{2 \pi}\right)} \\
c(\theta) & 0
\end{array}\right)
$$

is called the transfer matrix. Let

$$
A_{n}^{\lambda}(\theta)=A^{\lambda}\left(\theta+(n-1) \frac{\Phi}{2 \pi}\right) \cdots A^{\lambda}\left(\theta+\frac{\Phi}{2 \pi}\right) A^{\lambda}(\theta)
$$

be the $n$-step transfer matrix.

\footnotetext{
${ }^{7}$ For rational $\frac{\Phi}{2 \pi}$ and singular $H_{\Phi, \theta}, \Sigma_{\Phi, \theta}$ may consist of infinitely degenerate isolated eigenvalues, if $c$ vanishes somewhere on the orbit of rotation of $\theta$ by $\frac{\Phi}{2 \pi}$.

${ }^{8}$ In our concrete model, $c(\theta)=1+e^{-2 \pi i \theta}$, see (5.4), hence has a single zero $\theta_{1}=1 / 2$.
} 
We define the Lyapunov exponent of $H_{\Phi, \theta}$ at energy $\lambda$ as

$$
L(\lambda, \Phi):=\lim _{n \rightarrow \infty} \frac{1}{n} \int_{\mathbb{T}_{1}} \log \left\|A_{n}^{\lambda}(\theta)\right\| \mathrm{d} \theta .
$$

By a trivial bound $\|A\|^{2} \geq|\operatorname{det} A|$, which comes from the fact $A$ is a $2 \times 2$ matrix, we get

$$
L(\lambda, \Phi) \geq \lim _{n \rightarrow \infty} \frac{1}{2 n} \int_{\mathbb{T}_{1}} \log \left(\frac{\left|c\left(\theta-\frac{\Phi}{2 \pi}\right)\right|}{\mid c\left(\theta+(n-1) \frac{\Phi}{2 \pi} \mid\right.}\right) \mathrm{d} \theta=0 .
$$

\subsubsection{Normalized transfer matrix}

Let $|c(\theta)|=\sqrt{c(\theta) \overline{c(\theta)}}$. We introduce the normalized transfer matrix:

$$
\operatorname{SL}(2, \mathbb{R}) \ni \tilde{A}^{\lambda}(\theta)=\frac{1}{\sqrt{|c(\theta)|\left|c\left(\theta-\frac{\Phi}{2 \pi}\right)\right|}}\left(\begin{array}{cc}
\lambda-v(\theta)-\left|c\left(\theta-\frac{\Phi}{2 \pi}\right)\right| \\
|c(\theta)| & 0
\end{array}\right)
$$

and the $n$-step normalized transfer matrix $\tilde{A}_{n}^{\lambda}(\theta)$.

The following connection between $A^{\lambda}$ and $\tilde{A}^{\lambda}$ is clear:

$$
\tilde{A}^{\lambda}(\theta)=\frac{c(\theta)}{\sqrt{|c(\theta)|\left|c\left(\theta-\frac{\Phi}{2 \pi}\right)\right|}}\left(\begin{array}{ll}
1 & 0 \\
0 & \frac{0}{\mid c(\theta)}
\end{array}\right) A^{\lambda}(\theta)\left(\begin{array}{ll}
1 & \frac{0}{c(\theta) \mid} \\
0 & \frac{\bar{c}\left(\theta-\frac{\Phi}{2 \pi}\right)}{\left|c\left(\theta-\frac{\Phi}{2 \pi}\right)\right|}
\end{array}\right)^{-1} .
$$

When $\frac{\Phi}{2 \pi}=\frac{p}{q}$ is rational, (2.47) yields

$$
\operatorname{tr}\left(\tilde{A}_{q}^{\lambda}(\theta)\right)=\frac{\prod_{j=0}^{q-1} c\left(\theta+j \frac{p}{q}\right)}{\prod_{j=0}^{q-1}\left|c\left(\theta+j \frac{p}{q}\right)\right|} \operatorname{tr}\left(A_{q}^{\lambda}(\theta)\right) .
$$

Let

$$
D^{\lambda}(\theta)=c(\theta) A^{\lambda}(\theta)=\left(\begin{array}{cc}
\lambda-v(\theta) & -\overline{c\left(\theta-\frac{\Phi}{2 \pi}\right)} \\
c(\theta) & 0
\end{array}\right)
$$

and $D_{n}^{\lambda}(\theta)=D^{\lambda}\left(\theta+(n-1) \frac{\Phi}{2 \pi}\right) \cdots D^{\lambda}\left(\theta+\frac{\Phi}{2 \pi}\right) D^{\lambda}(\theta)$. Then when $\frac{\Phi}{2 \pi}=\frac{p}{q}$ is rational, (2.48) becomes

$$
\operatorname{tr}\left(\tilde{A}_{q}^{\lambda}(\theta)\right)=\frac{\operatorname{tr}\left(D_{q}^{\lambda}(\theta)\right)}{\prod_{j=0}^{q-1}\left|c\left(\theta+j \frac{p}{q}\right)\right|} .
$$


Note that although $A_{n}^{\lambda}(\theta)$ is not well-defined for $\theta \in \Theta, D_{n}^{\lambda}(\theta)$ is always well-defined.

\section{Magnetic Hamiltonians on quantum graphs}

\subsection{Magnetic potential}

Given a vector potential $A(\boldsymbol{x})=A_{1}\left(x_{1}, x_{2}\right) d x_{1}+A_{2}\left(x_{1}, x_{2}\right) d x_{2} \in \Omega^{1}\left(\mathbb{R}^{2}\right)$, the scalar potential $A_{\vec{e}} \in C^{\infty}(\vec{e})$ along edges $\vec{e} \in \mathcal{E}(\Lambda)$ is obtained by evaluating the form $A$ on the graph along the vector field generated by edges $[\vec{e}] \in \mathcal{E}\left(W_{\Lambda}\right)$

$$
A_{\vec{e}}(x):=A(x)\left([\vec{e}]_{1} \partial_{1}+[\vec{e}]_{2} \partial_{2}\right) .
$$

The integrated vector potentials are defined as $\beta_{\vec{e}}:=\int_{\vec{e}} A_{\vec{e}}(\boldsymbol{x}) d \boldsymbol{x}$ for $\vec{e} \in \mathcal{E}(\Lambda)$.

Assumption 1 The magnetic flux $\Phi$ through each hexagon $\oslash$ of the lattice

$$
\Phi:=\int_{\circlearrowright} d A
$$

is assumed to be constant.

Let us mention that the assumption above is equivalent to the following equation, in terms of the integrated vector potentials

$$
\begin{aligned}
& \beta_{\gamma_{1}, \gamma_{2}, \vec{f}}-\beta_{\gamma_{1}, \gamma_{2}+1, \vec{h}}+\beta_{\gamma_{1}, \gamma_{2}+1, \vec{g}}-\beta_{\gamma_{1}-1, \gamma_{2}+1, \vec{f}}+\beta_{\gamma_{1}-1, \gamma_{2}+1, \vec{h}}-\beta_{\gamma_{1}, \gamma_{2}, \vec{g}} \\
& \quad=\Phi
\end{aligned}
$$

for any $\gamma_{1}, \gamma_{2} \in \mathbb{Z}$.

Example 1 The vector potential $A \in \Omega^{1}\left(\mathbb{R}^{2}\right)$ of a homogeneous magnetic field $B \in \Omega^{2}\left(\mathbb{R}^{2}\right)$

$$
B(x)=B_{0} d x_{1} \wedge d x_{2}
$$

can be chosen as

$$
A(x):=B_{0} x_{1} d x_{2} .
$$

This scalar potential is invariant under $\vec{b}_{2}$-translations. The integrated vector potentials $\beta_{\vec{e}}$ are given by

$$
\beta_{\gamma_{1}, \gamma_{2}, \vec{f}}=\frac{\Phi}{2}\left(\gamma_{1}+\frac{1}{6}\right), \beta_{\gamma_{1}, \gamma_{2}, \vec{g}}=0, \text { and } \beta_{\gamma_{1}, \gamma_{2}, \vec{h}}=-\beta_{\gamma_{1}, \gamma_{2}, \vec{f}},
$$

where, in this case, the magnetic flux through each hexagon is $\Phi=\frac{3 \sqrt{3}}{2} B_{0}$. 


\subsection{Magnetic differential operator and modified Peierls' substitution}

In terms of the magnetic differential operator $\left(D^{B} \psi\right)_{\vec{e}}:=-i \psi_{\vec{e}}^{\prime}-A_{\vec{e}} \psi_{\vec{e}}$, the Schrödinger operator modeling graphene in a magnetic field reads

$$
\begin{aligned}
& H^{B}: D\left(H^{B}\right) \subset L^{2}(\mathcal{E}(\Lambda)) \rightarrow L^{2}(\mathcal{E}(\Lambda)) \\
& \left(H^{B} \psi\right)_{\vec{e}}:=\left(D^{B} D^{B} \psi\right)_{\vec{e}}+V_{\vec{e}} \psi_{\vec{e}},
\end{aligned}
$$

and is defined on

$$
\begin{gathered}
D\left(H^{B}\right):=\left\{\psi \in \mathcal{H}^{2}(\mathcal{E}(\Lambda)): \psi_{\vec{e}_{1}}(\boldsymbol{v})=\psi_{\vec{e}_{2}}(\boldsymbol{v}) \text { for any } \vec{e}_{1}, \vec{e}_{2} \in \mathcal{E}_{\boldsymbol{v}}(\Lambda)\right. \\
\text { and } \left.\sum_{\vec{e} \in \mathcal{E}_{\boldsymbol{v}}(\Lambda)}\left(D^{B} \psi\right)_{\vec{e}}(\boldsymbol{v})=0\right\} .
\end{gathered}
$$

Let us first introduce a unitary operator $U$ on $L^{2}(\mathcal{E}(\Lambda))$, defined as

$$
U \psi_{\gamma_{1}, \gamma_{2}, \vec{e}}=\zeta_{\gamma_{1}, \gamma_{2}} \psi_{\gamma_{1}, \gamma_{2}, \vec{e}} \text { for } \vec{e}=\vec{f}, \vec{g}, \vec{h},
$$

the factors $\zeta_{\gamma_{1}, \gamma_{2}}$ are defined as follows. First, choose a path $p(\cdot): \mathbb{N} \rightarrow \mathbb{Z}^{2}$ connecting $(0,0)$ to $\left(\gamma_{1}, \gamma_{2}\right)$ with

$$
p(0)=(0,0) \text { and } p\left(\left|\gamma_{1}\right|+\left|\gamma_{2}\right|\right)=\left(\gamma_{1}, \gamma_{2}\right) \text {. }
$$

Note that (3.10) implies that both components of $p(\cdot)$ are monotonic functions. Then we define $\zeta_{\gamma_{1}, \gamma_{2}}$ recursively through the following relations along $p(\cdot)$ :

$$
\begin{aligned}
\zeta_{0,0} & =1, \\
\zeta_{\gamma_{1}+1, \gamma_{2}} & =e^{i \beta_{\gamma_{1}, \gamma_{2}, \vec{f}}-i \beta_{\gamma_{1}+1, \gamma_{2}, \vec{g}}} \zeta_{\gamma_{1}, \gamma_{2}}, \\
\zeta_{\gamma_{1}, \gamma_{2}+1} & =e^{i \beta_{\gamma_{1}, \gamma_{2}, \vec{f}}-i \beta_{\gamma_{1}, \gamma_{2}+1, \vec{h}}-i \Phi \gamma_{1}} \zeta_{\gamma_{1}, \gamma_{2}} .
\end{aligned}
$$

Due to (3.3), it is easily seen that the definition of $\zeta_{\gamma_{1}, \gamma_{2}}$ is independent of the choice of $p(\cdot)$, hence is well-defined.

The unitary Peierls' substitution ${ }^{9}$ is the multiplication operator

$$
\begin{aligned}
P & : L^{2}(\mathcal{E}(\Lambda)) \rightarrow L^{2}(\mathcal{E}(\Lambda)) \\
& \left(\psi_{\vec{e}}\right) \mapsto\left(\left(\vec{e} \ni \boldsymbol{x} \mapsto e^{i \int_{i(\vec{e}) \rightarrow x} A_{\vec{e}}(s) d s}\right) \psi_{\vec{e}}\right),
\end{aligned}
$$

\footnotetext{
9 This transform is also known as minimal coupling.
} 
where $i(\vec{e}) \rightarrow \boldsymbol{x}$ denotes the straight line connecting $i(\vec{e})$ with $x \in \vec{e}$. It reduces the magnetic Schrödinger operator to non-magnetic ones with magnetic contribution moved into boundary condition, with multiplicative factors at terminal edges given by $e^{i \beta_{\vec{e}}}$.

We will define a modified Peierls' substitution that allows us to reduce the number of non-trivial multiplicative factors to one, by taking

$$
\tilde{P}=P U
$$

It transforms $H^{B}$ into

$$
\Lambda^{B}:=\left(-\frac{d^{2}}{d t_{\vec{e}}^{2}}+V_{\vec{e}}\right)_{\vec{e} \in \mathcal{E}(\Lambda)}=\tilde{P}^{-1} H^{B} \tilde{P} .
$$

The domain of $\Lambda^{B}$ is

$$
\begin{aligned}
D\left(\Lambda^{B}\right)=\{ & \psi \in \mathcal{H}^{2}(\mathcal{E}(\Lambda)): \text { any } \vec{e}_{1}, \vec{e}_{2} \in \mathcal{E}(\Lambda) \text { with } i\left(\vec{e}_{1}\right)=i\left(\vec{e}_{2}\right)=\boldsymbol{v} \text { satisfy } \\
& \psi_{\vec{e}_{1}}(\boldsymbol{v})=\psi_{\vec{e}_{2}}(\boldsymbol{v}) \text { and } \sum_{i(\vec{e})=v} \psi_{\vec{e}}^{\prime}(\boldsymbol{v})=0 \text {; whilst at edges for which } \\
& t\left(\vec{e}_{1}\right)=t\left(\vec{e}_{2}\right)=\boldsymbol{v}, e^{i \tilde{\beta}_{\vec{e}_{1}}} \psi_{\vec{e}_{1}}(\boldsymbol{v})=e^{i \tilde{\beta}_{\vec{e}_{2}}} \psi_{\vec{e}_{2}}(\boldsymbol{v}) \text { and } \\
& \left.\sum_{t(\vec{e})=v} e^{i \tilde{\beta}_{\vec{e}}} \psi_{\vec{e}}^{\prime}(\boldsymbol{v})=0\right\},
\end{aligned}
$$

where

$$
\tilde{\beta}_{\gamma_{1}, \gamma_{2}, \vec{g}} \equiv \tilde{\beta}_{\gamma_{1}, \gamma_{2}, \vec{f}} \equiv 0 \text { and } \tilde{\beta}_{\gamma_{1}, \gamma_{2}, \vec{h}}=-\Phi \gamma_{1} \text {. }
$$

Thus, the problem reduces to the study of non-magnetic Schrödinger operators with magnetic contributions moved into the boundary conditions.

Observe that the magnetic Dirichlet operator

$$
\begin{aligned}
& H^{D, B}: \bigoplus_{\vec{e} \in \mathcal{E}(\Lambda)}\left(\mathcal{H}_{0}^{1}(\vec{e}) \cap \mathcal{H}^{2}(\vec{e})\right) \subset L^{2}(\mathcal{E}(\Lambda)) \rightarrow L^{2}(\mathcal{E}(\Lambda)) \\
& \left(H^{D, B} \psi\right)_{\vec{e}}:=\left(D^{B} D^{B} \psi\right)_{\vec{e}}+V_{\vec{e}} \psi_{\vec{e}}
\end{aligned}
$$

is by the (modified) Peierls' substitution unitary equivalent to the Dirichlet operator without magnetic field

$$
H^{D}=\tilde{P}^{-1} H^{D, B} \tilde{P}=P^{-1} H^{D, B} P .
$$


Consequently, the spectrum of the Dirichlet operator $H^{D}$ is invariant under perturbations by the magnetic field.

\section{Main lemmas}

First, let us introduce the following two-dimensional tight-binding Hamiltonian

$$
Q_{\Lambda}(\Phi):=\frac{1}{3}\left(\begin{array}{cc}
0 & 1+\tau_{0}+\tau_{1} \\
\left(1+\tau_{0}+\tau_{1}\right)^{*} & 0
\end{array}\right)
$$

with translation operators $\tau_{0}, \tau_{1} \in \mathcal{L}\left(l^{2}\left(\mathbb{Z}^{2} ; \mathbb{C}\right)\right)$ which for $\gamma \in \mathbb{Z}^{2}$ and $u \in$ $l^{2}\left(\mathbb{Z}^{2} ; \mathbb{C}\right)$ are defined as

$$
\left(\tau_{0}(u)\right)_{\gamma_{1}, \gamma_{2}}:=u_{\gamma_{1}-1, \gamma_{2}} \text { and }\left(\tau_{1}(u)\right)_{\gamma_{1}, \gamma_{2}}:=e^{-i \Phi \gamma_{1}} u_{\gamma_{1}, \gamma_{2}-1} .
$$

The following lemma connects the spectrum of $H^{B}$ with $\sigma\left(Q_{\Lambda}\right)$. We have Lemma 4.1 Let $\Delta(\lambda)$ be the Hill discriminant defined in (2.37). A number $\lambda \in \rho\left(H^{D}\right)$ lies in $\sigma\left(H^{B}\right)$ iff $\Delta(\lambda) \in \sigma\left(Q_{\Lambda}(\Phi)\right)$. Such $\lambda$ is in the point spectrum of $H^{B}$ iff $\Delta(\lambda) \in \sigma_{p}\left(Q_{\Lambda}(\Phi)\right)$.

Remark 4 We will show in Lemma 5.2 that $\sigma_{p}\left(Q_{\Lambda}(\Phi)\right)$ is empty, thus $H^{B}$ has no point spectrum away from $\sigma\left(H^{D}\right)$.

Lemma 4.2 below shows $\sigma\left(Q_{\Lambda}(\Phi)\right)$ is a zero-measure Cantor set for irrational flux $\frac{\Phi}{2 \pi}$, Lemma 4.3 gives a measure estimate for rational flux, and Lemma 4.4 provides an upper bound on the Hausdorff dimension of the spectrum of $Q_{\Lambda}(\Phi)$. These three lemmas prove the topological structure part of Theorem 3.

Lemma 4.2 For $\frac{\Phi}{2 \pi} \in \mathbb{R} \backslash \mathbb{Q}, \sigma\left(Q_{\Lambda}(\Phi)\right)$ is a zero-measure Cantor set.

Lemma 4.3 If $\frac{\Phi}{2 \pi}=\frac{p}{q}$ is a reduced rational number, then $\sigma\left(Q_{\Lambda}(\Phi)\right)$ is a finite union of intervals, with measure estimate

$$
\left|\sigma\left(Q_{\Lambda}(\Phi)\right)\right| \leq \frac{8 \sqrt{6 \pi}}{9 \sqrt{q}} .
$$

Lemma 4.4 For generic $\Phi$, the Hausdorff dimension of $\sigma\left(Q_{\Lambda}(\Phi)\right)$ is $\leq \frac{1}{2}$.

\section{Reduction of $Q_{\Lambda}(\Phi)$ to a one-dimensional Jacobi matrix}

\subsection{Symmetric property of $Q_{\Lambda}$}

Lemma 5.1 The spectrum of $Q_{\Lambda}$ has the following properties: 
(1) $\sigma\left(Q_{\Lambda}(\Phi)\right)$ is symmetric with respect to 0 .

(2) $0 \in \sigma\left(Q_{\Lambda}(\Phi)\right)$.

Proof (1). Conjugating $Q_{\Lambda}$ in (4.1) by

$$
\Omega=\left(\begin{array}{cc}
-\mathrm{id} & 0 \\
0 & \mathrm{id}
\end{array}\right)
$$

shows that $\sigma\left(Q_{\Lambda}(\Phi)\right)$ is symmetric with respect to 0 [40, Prop. 3.5].

(2). If we view $Q_{\Lambda}(\Phi)$ as an operator-valued function of the flux $\Phi$, then

$$
\Phi \mapsto\left\langle Q_{\Lambda}(\Phi) x, y\right\rangle
$$

for $x, y \in c_{00}$ arbitrary, is analytic and $Q_{\Lambda}$ therefore is a bounded analytic map. If there was $\Phi_{0} / 2 \pi \in \mathbb{R} \backslash \mathbb{Q}$ where $Q_{\Lambda}\left(\Phi_{0}\right)$ was invertible, then $Q_{\Lambda}(\Phi)$ would also be invertible in a sufficiently small neighborhood of $\Phi_{0}$ (e.g. [38, Ch.7.1]). Yet, in [28, Prop.4.1] it has been shown that for rational $\Phi / 2 \pi$, $0 \in \sigma\left(Q_{\Lambda}(\Phi)\right)$. Thus, by density $0 \in \sigma\left(Q_{\Lambda}(\Phi)\right)$, independent of $\Phi \in \mathbb{R}$.

\subsection{Reduction to the one-dimensional Hamiltonian}

Relating the spectrum of $Q_{\Lambda}$ to that of $Q_{\Lambda}^{2}$, we obtain the following characterization of $\sigma\left(Q_{\Lambda}\right)$.

Lemma 5.2 (1) The spectrum of the operator $Q_{\Lambda}(\Phi)$ as a set is given by

$$
\sigma\left(Q_{\Lambda}(\Phi)\right)= \pm \sqrt{\frac{\bigcup_{\theta \in \mathbb{T}_{1}} \sigma\left(H_{\Phi, \theta}\right)}{9}+\frac{1}{3}} \bigcup\{0\}
$$

where $H_{\Phi, \theta} \in \mathcal{L}\left(l^{2}(\mathbb{Z})\right)$ is the one-dimensional quasi-periodic Jacobi matrix defined as in (2.41) with

$$
c(\theta)=1+e^{-2 \pi i \theta}, \text { and } v(\theta)=2 \cos 2 \pi \theta .
$$

(2) $Q_{\Lambda}(\Phi)$ has no point spectrum.

Proof (1). Let $A:=\frac{1}{3}\left(1+\tau_{0}+\tau_{1}\right)$. Then squaring the operator $Q_{\Lambda}(\Phi)$ yields

$$
Q_{\Lambda}^{2}(\Phi)=\left(\begin{array}{cc}
A A^{*} & 0 \\
0 & A^{*} A
\end{array}\right)
$$

The spectral mapping theorem implies that $\sigma\left(Q_{\Lambda}^{2}(\Phi)\right)=\sigma\left(Q_{\Lambda}(\Phi)\right)^{2}$ and from Lemma 5.2 we conclude that $\sigma\left(Q_{\Lambda}(\Phi)\right)= \pm \sqrt{\sigma\left(Q_{\Lambda}^{2}(\Phi)\right)}$. Clearly, 
the operator $\left.A A^{*}\right|_{\operatorname{ker}\left(A^{*}\right)^{\perp}}$ and $\left.A^{*} A\right|_{\operatorname{ker}(A)^{\perp}}$ are unitarily equivalent. Thus, the spectrum can be expressed by

$$
\sigma\left(Q_{\Lambda}(\Phi)\right)= \pm \sqrt{\sigma\left(A A^{*}\right)} \cup\{0\}
$$

where we are able to use either of the two $\left(A A^{*}\right.$ or $\left.A^{*} A\right)$ since $0 \in \sigma\left(Q_{\Lambda}(\Phi)\right)$ due to Lemma 5.1.

Then, it follows that

$$
A A^{*}=\frac{\mathrm{id}}{3}+\frac{\mathrm{id}}{9} \underbrace{\left(\left(\tau_{0}+\tau_{0}^{*}\right)+\left(\tau_{1}+\tau_{1}^{*}\right)+\tau_{0} \tau_{1}^{*}+\tau_{1} \tau_{0}^{*}\right)}_{=: H_{\Phi}} .
$$

Observe that

$$
\begin{aligned}
H_{\Phi} \psi_{m, n}= & \psi_{m-1, n}+\psi_{m+1, n}+e^{-i \Phi m} \psi_{m, n-1}+e^{i \Phi m} \psi_{m, n+1} \\
& +e^{i \Phi(m-1)} \psi_{m-1, n+1}+e^{-i \Phi m} \psi_{m+1, n-1} .
\end{aligned}
$$

Since $H_{\Phi}$ is invariant under discrete translations in $n$, the operator is unitarily equivalent to the direct integral operator $\int_{\mathbb{T}_{1}}^{\oplus} H_{\Phi, \theta} d \theta$, which gives the claim.

(2). It follows from a standard argument that the two dimensional operator $H_{\Phi}$ has no point spectrum. Indeed, assume $H_{\Phi}$ has point spectrum at energy $E$, then $H_{\Phi, \theta}$ would have the same point spectrum $E$ for a.e. $\theta \in \mathbb{T}_{1}$. This implies the integrated density of states of $H_{\Phi, \theta}$ has a jump discontinuity at $E$, which is impossible. Therefore the point spectrum of $H_{\Phi}$ is empty, hence the same holds for $Q_{\Lambda}(\Phi)$.

\section{Proof of Lemmas 4.2, 4.3 and 4.4}

For a set $U$, let $\operatorname{dim}_{H}(U)$ be its Hausdorff dimension.

Lemma 4.2 follows as a direct consequence of (5.3) and the following Theorem 7. Let $\Sigma_{\Phi}$ be defined as in Sect. 2.3.

Theorem 7 For $\frac{\Phi}{2 \pi} \in \mathbb{R} \backslash \mathbb{Q}, \Sigma_{\Phi}$ is a zero-measure Cantor set.

We will postpone the proof of Theorem 7 till the end of this section. We will first present the proofs of Lemmas 4.3 and 4.4, which are based on the following three lemmas. First, we have

Lemma 6.1 Let $\frac{\Phi}{2 \pi}=\frac{p}{q}$ be a reduced rational number, then $\Sigma_{2 \pi p / q}$ is a union of $q$ (possibly touching) bands with $\left|\Sigma_{2 \pi p / q}\right|<\frac{16 \pi}{3 q}$.

Lemma 6.1 will be proved in Sects. 6.4 and 6.5 after some further preparation. The following lemma addresses the continuity of the spectrum $\Sigma_{\Phi}$ in 
$\Phi$, extending a result of [9] (see Proposition 7.1 therein) from quasiperiodic Schrödinger operators to Jacobi matrices.

Lemma 6.2 There exist absolute constants $C_{1}, C_{2}>0$ such that if $\lambda \in \Sigma_{\Phi}$ and $\left|\Phi-\Phi^{\prime}\right|<C_{1}$, then there exists $\lambda^{\prime} \in \Sigma_{\Phi^{\prime}}$ such that

$$
\left|\lambda-\lambda^{\prime}\right| \leq C_{2}\left|\Phi-\Phi^{\prime}\right|^{\frac{1}{2}}
$$

We will prove Lemma 6.2 in "Appendix C".

The next lemma provides an upper bound on the Hausdorff dimension of a set.

Lemma 6.3 (Lemma 5.1 of [42]) Let $S \subset \mathbb{R}$, and suppose that $S$ has a sequence of covers: $\left\{S_{n}\right\}_{n=1}^{\infty}, S \subset S_{n}$, such that each $S_{n}$ is a union of $q_{n}$ intervals, $q_{n} \rightarrow \infty$ as $n \rightarrow \infty$, and for each $n$,

$$
\left|S_{n}\right|<\frac{C}{q_{n}^{\beta}},
$$

where $\beta$ and $C$ are positive constants, then

$$
\operatorname{dim}_{H}(S) \leq \frac{1}{1+\beta}
$$

\section{Proof of Lemma 4.3}

The fact that $\sigma\left(Q_{\Lambda}(\Phi)\right)$ is a finite union of intervals follows from (5.3) and Lemma 6.1.

It suffices to prove the measure estimate. It is clear that for any $\varepsilon>0$, we have

$$
\sqrt{\Sigma_{2 \pi p / q}+3} \subseteq[0, \sqrt{\varepsilon}] \bigcup \sqrt{\left(\Sigma_{2 \pi p / q}+3\right) \bigcap(\varepsilon, \infty)} .
$$

Hence by Lemma 6.1, we have

$$
\left|\sqrt{\Sigma_{2 \pi p / q}+3}\right| \leq \sqrt{\varepsilon}+\frac{\left|\Sigma_{2 \pi p / q}\right|}{2 \sqrt{\varepsilon}} \leq \sqrt{\varepsilon}+\frac{8 \pi}{3 \sqrt{\varepsilon} q} .
$$

Optimizing in $\varepsilon$ leads to

$$
\left|\sqrt{\Sigma_{2 \pi p / q}+3}\right| \leq \frac{4 \sqrt{6 \pi}}{3 \sqrt{q}}
$$


Then (5.3) implies

$$
\left|\sigma\left(Q_{\Lambda}(2 \pi p / q)\right)\right| \leq \frac{8 \sqrt{6 \pi}}{9 \sqrt{q}} .
$$

\section{Proof of Lemma 4.4}

We will show that if $\frac{\Phi}{2 \pi}$ is an irrational obeying

$$
q_{n}^{4}\left|\frac{\Phi}{2 \pi}-\frac{p_{n}}{q_{n}}\right|<C
$$

for some constant $C$, and a sequence of reduced rationals $\left\{p_{n} / q_{n}\right\}$ with $q_{n} \rightarrow$ $\infty$, then $\operatorname{dim}_{H}\left(\sigma\left(Q_{\Lambda}(\Phi)\right)\right) \leq 1 / 2$. It is easy to see that the $\Phi$ 's satisfying (6.2) form a dense $G_{\delta}$ set of $\mathbb{R}$, hence is generic.

Without loss of generality, we may assume $\frac{\Phi}{2 \pi} \in(0,1)$.

First, by (5.3), we have that

$$
\operatorname{dim}_{H}\left(\sigma\left(Q_{\Lambda}(\Phi)\right)\right)=\sup _{k \geq 2} \operatorname{dim}_{H}\left( \pm \sqrt{\left(\frac{\Sigma_{\Phi}}{9}+\frac{1}{3}\right) \cap\left[\frac{1}{k}, 1\right]}\right)
$$

where we used a trivial bound $\left\|H_{\Phi, \theta}\right\| \leq 6$. Hence it suffices to show that for each $k \geq 2$,

$$
\operatorname{dim}_{H}\left(\sqrt{\left(\frac{\Sigma_{\Phi}}{9}+\frac{1}{3}\right) \cap\left[\frac{1}{k}, 1\right]}\right) \leq \frac{1}{2} .
$$

The rest of the argument is similar to that of [42]. By Lemma 6.2, taking any $\lambda \in \Sigma_{\Phi}$, for $n \geq n_{0}$, there exists $\lambda^{\prime} \in \Sigma_{2 \pi p_{n} / q_{n}}$ such that $\left|\lambda-\lambda^{\prime}\right| \leq$ $C_{2}\left|\frac{\Phi}{2 \pi}-\frac{p_{n}}{q_{n}}\right|^{\frac{1}{2}}$. This means $\Sigma_{\Phi}$ is contained in the $C_{2}\left|\frac{\Phi}{2 \pi}-\frac{p_{n}}{q_{n}}\right|^{\frac{1}{2}}$ neighbourhood of $\Sigma_{2 \pi p_{n} / q_{n}}$. By Lemma 6.1, $\Sigma_{2 \pi p_{n} / q_{n}}$ has $q_{n}$ (possibly touching) bands with total measure $\left|\Sigma_{2 \pi p_{n} / q_{n}}\right| \leq \frac{16 \pi}{3 q_{n}}$. Hence $\Sigma_{\Phi}$ has cover $S_{n}$ such that $S_{n}$ is a union of (at most) $q_{n}$ intervals with total measure

$$
\left|S_{n}\right| \leq \frac{16 \pi}{3 q_{n}}+2 C_{2} q_{n}\left|\frac{\Phi}{2 \pi}-\frac{p_{n}}{q_{n}}\right|^{\frac{1}{2}} .
$$


Since $q_{n}^{4}\left|\frac{\Phi}{2 \pi}-\frac{p_{n}}{q_{n}}\right| \leq C$, we have, by (6.4),

$$
\left|S_{n}\right| \leq \frac{16 \pi}{3 q_{n}}+\frac{2 C_{2} \sqrt{C}}{q_{n}}=: \frac{\tilde{C}}{q_{n}} .
$$

This implies $\left(\frac{\Sigma_{\Phi}}{9}+\frac{1}{3}\right) \cap\left[\frac{1}{k}, 1\right]$ has cover $\tilde{S}_{n}$ such that $\tilde{S}_{n}$ is a union of (at most) $q_{n}$ intervals with total measure

$$
\left|\tilde{S}_{n}\right| \leq \frac{\sqrt{k} \tilde{C}}{2 q_{n}} .
$$

Then Lemma 6.3 yields (6.3).

\subsection{Proof of Theorem 7}

Note that Lemmas 6.1 and 6.2 already imply zero measure (and thus Cantor nature) of the spectrum for fluxes $\Phi / 2 \pi$ with unbounded coefficients in the continued fraction expansion, thus for a.e. $\Phi$, by an argument similar to that used in the proof of Lemma 4.4. However extending the result to the remaining measure zero set this way would require a slightly stronger continuity in Lemma 6.2, which is not available. We circumvent this by the following strategy:

(1). Use quantization of acceleration techniques to prove the Lyapunov exponent of operator $H_{\Phi, \theta}$ identically vanishes on the spectrum, see Proposition 6.4;

(2). employ the singularity of the Jacobi matrix to show the absolutely continuous spectrum of $H_{\Phi, \theta}$ is empty, see Proposition 6.5;

(3). apply Kotani theory for Jacobi matrices, see Theorem 8.

Let $\Sigma_{a c}\left(H_{\Phi, \theta}\right)$ be the absolutely continuous spectrum of $H_{\Phi, \theta}$. Let $L(\lambda, \Phi)$ be the Lyapunov exponent of $H_{\Phi, \theta}$ at energy $\lambda$, as defined in (2.44). For a set $U \subseteq \mathbb{R}$, let $\bar{U}^{e s s}$ be its essential closure.

First, we are able to give a characterization of the Lyapunov exponent on the spectrum.

Proposition 6.4 For $\frac{\Phi}{2 \pi} \in \mathbb{R} \backslash \mathbb{Q}, L(\lambda, \Phi)=0$ if and only if $\lambda \in \Sigma_{\Phi}$.

The proof of this is similar to that for the almost Mathieu operator as given in [2] and the extended Harper's model [34]. The general idea is to complexify $\theta$ to $\theta+i \varepsilon$, and obtain asymptotic behavior of the Lyapunov exponent when $|\varepsilon| \rightarrow$ $\infty$. Convexity and quantization of the acceleration (see Theorem 5 of [2]) then bring us back to the $\varepsilon=0$ case. We will give the proof in "Appendix A".

Exploiting the fact that $c(\theta)=1+e^{-2 \pi i \theta}$ has a real zero $\theta_{1}=\frac{1}{2}$, we have 
Proposition 6.5 ([18], see also Proposition 7.1 of [34]) For $\frac{\Phi}{2 \pi} \in \mathbb{R} \backslash \mathbb{Q}$, and a.e. $\theta \in \mathbb{T}_{1}, \Sigma_{a c}\left(H_{\Phi, \theta}\right)$ is empty.

Hence our operator $H_{\Phi, \theta}$ has zero Lyapunov exponent on the spectrum and empty absolutely continuous spectrum. Celebrated Kotani theory identifies the essential closure of the set of zero Lyapunov exponents with the absolutely continuous spectrum, for general ergodic Schrödinger operators. This has been extended to the case of non-singular (that is $|c(\cdot)|$ uniformly bounded away from zero) Jacobi matrices in Theorem 5.17 of [52]. In our case $|c(\cdot)|$ is not bounded away from zero, however a careful inspection of the proof of Theorem 5.17 of [52] shows that it holds under a weaker requirement: $\log (|c(\cdot)|) \in L^{1}$. Namely, let $H_{c, v}(\theta)$ acting on $\ell^{2}(\mathbb{Z})$ be an ergodic Jacobi matrix,

$$
\left(H_{c, v}(\theta) u\right)_{m}=c\left(T^{m} \theta\right) u_{m+1}+\overline{c\left(T^{m-1} \theta\right)} u_{m-1}+v\left(T^{m} \theta\right) u_{m}
$$

where $c: M \rightarrow \mathbb{C}, v: M \rightarrow \mathbb{R}$, are bounded measurable functions, and $T: M \rightarrow M$ is an ergodic map. Let $L_{c, v}(\lambda)$ be the corresponding Lyapunov exponent. We have

Theorem 8 (Kotani theory) Assume $\log (|c(\cdot)|) \in L^{1}(M)$. Then for a.e. $\theta \in$ $M, \Sigma_{a c}\left(H_{c, v}(\theta)\right)={\overline{\left\{\lambda: L_{c, v}(\lambda)=0\right\}}}^{\text {ess }}$.

Proof The proof of Theorem 5.17 of [52] works verbatim.

Proof of Theorem 7 In our concrete model, $\log (|c(\theta)|)=\log (2|\cos \pi \theta|) \in$ $L^{1}\left(\mathbb{T}_{1}\right)$, thus Theorem 8 applies, and combining with Propositions 6.4, 6.5, it follows that $\Sigma_{\Phi}$ must be a zero measure set.

The rest of this section will be devoted to proving Lemma 6.1.

\subsection{Quick observations about $H_{2 \pi p / q, \theta}$}

Let $A^{\lambda}(\cdot), \tilde{A}^{\lambda}(\cdot), D^{\lambda}(\cdot), \Theta$ be defined as in Sect. 2.3.1. We start with several quick observations about $H_{2 \pi p / q, \theta}$.

Obervation 1 The sampling function $c(\theta)=0$ yields a unique solution $\theta=\frac{1}{2}$ $(\bmod 1)$, hence $\Theta=\frac{1}{2}+\frac{1}{q} \mathbb{Z}$. Then,

- for $\theta \notin \Theta$, we have $c\left(\theta+n \frac{p}{q}\right) \neq 0$ for any $n \in \mathbb{Z}$

- for $\theta \in \Theta$, there exists $k_{0} \in\{0,1, \ldots, q-1\}$ such that $c\left(\theta+n \frac{p}{q}\right)=0$ if and only if $n \equiv k_{0}(\bmod q)$. 
Note that $|c(\theta)|=2|\cos \pi \theta|$, so a simple computation yields that $\prod_{j=0}^{q-1}\left|c\left(\theta+j \frac{p}{q}\right)\right|=2\left|\sin \pi q\left(\theta+\frac{1}{2}\right)\right|$. Thus (2.50) becomes

$$
\operatorname{tr}\left(\tilde{A}_{q}^{\lambda}(\theta)\right)=\frac{\operatorname{tr}\left(D_{q}^{\lambda}(\theta)\right)}{2\left|\sin \pi q\left(\theta+\frac{1}{2}\right)\right|} .
$$

We have the following characterization of $\Sigma_{2 \pi p / q, \theta}$.

\subsubsection{Case 1.}

If $\theta \in \Theta$, we have the following

Obervation 2 For $\theta \in \Theta$, the infinite matrix $H_{2 \pi p / q, \theta}$ is decoupled into copies of the following block matrix $M_{q}$ of size $q$ :

$$
\left(\begin{array}{cccc}
\frac{v\left(\frac{1}{2}+\frac{p}{q}\right)}{c\left(\frac{1}{2}+\frac{p}{q}\right)} & v\left(\frac{1}{2}+\frac{p}{q}\right) & & \\
\ddots & & & \\
& & \ddots & \\
& & \frac{v\left(\frac{1}{2}+(q-1) \frac{p}{q}\right)}{c\left(\frac{1}{2}+(q-1) \frac{p}{q}\right)} & v\left(\frac{1}{2}\right)
\end{array}\right)
$$

Thus

$$
\Sigma_{2 \pi p / q, \theta}=\left\{\text { eigenvalues of } M_{q}\right\}, \text { for } \theta \in \Theta
$$

\subsubsection{Case 2.}

If $\theta \notin \Theta$, by Floquet theory, we have

$$
\Sigma_{2 \pi p / q, \theta}=\left\{\lambda:\left|\operatorname{tr} \tilde{A}_{q}^{\lambda}(\theta)\right| \leq 2\right\} .
$$

Furthermore, the set $\left\{\lambda: \operatorname{tr} \tilde{A}_{q}^{\lambda}(\theta)=2 \cos 2 \pi \nu\right\}$ contains $q$ individual points (counting multiplicities), which are eigenvalues of the following $q \times q$ matrix $M_{q, v}$ : 


$$
M_{q, v}(\theta)=\left(\begin{array}{ccccc}
v\left(\theta+\frac{p}{q}\right) & \left|c\left(\theta+\frac{p}{q}\right)\right| & & e^{2 \pi i v}|c(\theta)| \\
\left|c\left(\theta+\frac{p}{q}\right)\right| & v\left(\theta+2 \frac{p}{q}\right) & \ddots & & \\
& \ddots & \ddots & \\
& & & & \\
& & & v\left(\theta+(q-1) \frac{p}{q}\right) & \left|c\left(\theta+(q-1) \frac{p}{q}\right)\right| \\
e^{-2 \pi i v}|c(\theta)| & & & \left|c\left(\theta+(q-1) \frac{p}{q}\right)\right| & v(\theta)
\end{array}\right)
$$

Combining (6.10) with (6.7), we arrive at an alternative representation

$$
\Sigma_{2 \pi p / q, \theta}=\left\{\lambda:\left|\operatorname{tr}\left(D_{q}^{\lambda}(\theta)\right)\right| \leq 4\left|\sin \pi q\left(\theta+\frac{1}{2}\right)\right|\right\} .
$$

\subsection{Key lemmas}

Let

$$
d_{q}(\theta)=\operatorname{tr}\left(D_{q}^{\lambda}(\theta)\right)
$$

We have

Lemma 6.6 (Chambers' type formula) For all $\theta \in \mathbb{T}_{1}$, we have

$$
d_{q}(\theta)=-2 \cos 2 \pi q \theta+G_{q}(\lambda)
$$

where $G_{q}(\lambda)$ (defined by (6.14)) is independent of $\theta$.

Remark 5 Chambers' formula is well-known for the celebrated almost Mathieu operator. It was also recently developed for various models including the tight-binding model $Q_{\Lambda}(\Phi)$ in [28]. Here we do not use the Chambers' formula for $Q_{\Lambda}(\Phi)$, rather we develop one for one-dimensional Hamiltonian $H_{\Phi, \theta}$.

Proof It is easily seen that $d_{q}(\cdot)$ is a $1 / q$-periodic function, thus

$$
d_{q}(\theta)=G_{q}(\lambda)+a_{q} e^{2 \pi i q \theta}+a_{-q} e^{-2 \pi i q \theta},
$$

in which the $G_{q}(\lambda)$ part is independent of $\theta$. One can easily compute the coefficients $a_{q}, a_{-q}$, and get $a_{q}=a_{-q}=-1$.

Lemma 6.7 For $\theta \in \Theta$,

$$
\operatorname{det}\left(\lambda \cdot I d-M_{q}(\theta)\right)=\operatorname{tr}\left(D_{q}^{\lambda}(\theta)\right)
$$


The proof of this lemma is stated in "Appendix B".

Combining (6.12), (6.9) and Lemma 6.7 with the fact that $\left|\sin \pi q\left(\theta+\frac{1}{2}\right)\right|=$ 0 for $\theta \in \Theta$, we arrive at

$$
\Sigma_{2 \pi p / q, \theta}=\left\{\lambda:\left|\operatorname{tr}\left(D_{q}^{\lambda}(\theta)\right)\right| \leq 4\left|\sin \pi q\left(\theta+\frac{1}{2}\right)\right|\right\}
$$

holds uniformly for $\theta \in \mathbb{T}_{1}$.

By (6.14), we get the following alternative characterization of $\Sigma_{2 \pi p / q, \theta}$.

$$
\begin{aligned}
\Sigma_{2 \pi p / q, \theta} & =\left\{\lambda:-4\left|\sin \pi q\left(\theta+\frac{1}{2}\right)\right|+2 \cos 2 \pi q \theta \leq G_{q}(\lambda)\right. \\
& \left.\leq 4\left|\sin \pi q\left(\theta+\frac{1}{2}\right)\right|+2 \cos 2 \pi q \theta\right\} .
\end{aligned}
$$

Let us denote $L_{q}(\theta):=4\left|\sin \pi q\left(\theta+\frac{1}{2}\right)\right|+2 \cos 2 \pi q \theta$, and $l_{q}(\theta):=$ $-4\left|\sin \pi q\left(\theta+\frac{1}{2}\right)\right|+2 \cos 2 \pi q \theta$. Then (6.17) translates into

$$
\Sigma_{2 \pi p / q, \theta}=\left\{\lambda: l_{q}(\theta) \leq G_{q}(\lambda) \leq L_{q}(\theta)\right\} .
$$

This clearly implies

$$
\Sigma_{2 \pi p / q}=\left\{\lambda: \min _{\mathbb{T}_{1}} l_{q}(\theta) \leq G_{q}(\lambda) \leq \max _{\mathbb{T}_{1}} L_{q}(\theta)\right\} .
$$

Note that $G_{q}(\lambda)$ is a polynomial of $\lambda$ of degree $q$ with leading coefficient $1, \Sigma_{2 \pi p / q}$ consists of $q$ non-overlapping (but possibly touching) bands and $G^{\prime}(\lambda) \neq 0$ in the interior of each band, see e.g. Section 6 of [28].

The following lemma provides estimates of $\left|\Sigma_{2 \pi p / q, \theta}\right|$ and holds for any Jacobi matrix (2.41).

Lemma 6.8 We have

$$
\left|\Sigma_{2 \pi p / q, \theta}\right| \leq 4|c(\theta)| .
$$

Proof For $\theta \in \Theta$, by (6.9), $\left|\Sigma_{2 \pi p / q, \theta}\right|=0$. It then suffices to consider $\theta \notin \Theta$.

By (6.10), we have

$$
\Sigma_{\frac{2 \pi p}{q}, \theta}=\left\{\lambda:-2 \leq \operatorname{tr}\left(\tilde{A}_{q}^{\lambda}(\theta)\right) \leq 2\right\} .
$$

Note that $\operatorname{tr}\left(\tilde{A}_{q}^{\lambda}(\theta)\right)$ is a polynomial of degree $q$ in $\lambda$ with leading coefficient 1. By standard Floquet theory, see e.g. [52,53], we have

$$
\frac{d}{d \lambda} \operatorname{tr}\left(\tilde{A}_{q}^{\lambda}(\theta)\right) \neq 0
$$


holds for any $\lambda$ such that $\operatorname{tr}\left(\tilde{A}_{q}^{\lambda}(\theta)\right) \in(-2,2)$. Hence $\Sigma_{2 \pi p / q, \theta}$ is completely determined by the $\lambda$ 's such that $\operatorname{tr}\left(\tilde{A}_{q}^{\lambda}(\theta)\right)= \pm 2$. By (6.10) and the explanation below it,

$$
\left\{\begin{array}{l}
\left\{\lambda: \operatorname{tr}\left(\tilde{A}_{q}^{\lambda}(\theta)\right)=2\right\}=\left\{\text { eigenvalues of } M_{q, 0}(\theta)\right\} \\
\left\{\lambda: \operatorname{tr}\left(\tilde{A}_{q}^{\lambda}(\theta)\right)=-2\right\}=\left\{\text { eigenvalues of } M_{q, \frac{1}{2}}(\theta)\right\}
\end{array}\right.
$$

Let $\left\{\lambda_{i}(\theta)\right\}_{i=1}^{q}$ be eigenvalues of $M_{q, 0}(\theta)$, labelled in the increasing order. Let $\left\{\tilde{\lambda}_{i}(\theta)\right\}_{i=1}^{q}$ be eigenvalues of $M_{q, \frac{1}{2}}(\theta)$, labelled also in the increasing order. Then we have

$$
\begin{aligned}
\left|\Sigma_{\frac{2 \pi p}{q}, \theta}\right| & =\sum_{k=1}^{q}(-1)^{q-k}\left(\lambda_{k}(\theta)-\tilde{\lambda}_{k}(\theta)\right) \\
& =\sum_{k=1}^{\left[\frac{q+1}{2}\right]}\left(\lambda_{q-2 k+2}(\theta)-\tilde{\lambda}_{q-2 k+2}(\theta)\right) \\
& -\sum_{k=1}^{\left[\frac{q-1}{2}\right]}\left(\lambda_{q-2 k+1}(\theta)-\tilde{\lambda}_{q-2 k+1}(\theta)\right) .
\end{aligned}
$$

Note the coefficient of $\left(\lambda_{q}(\theta)-\tilde{\lambda}_{q}(\theta)\right)$ is 1 rather than -1 . This is due to the fact that the leading coefficient of $\operatorname{tr}\left(\tilde{A}_{q}^{\lambda}(\theta)\right)$ is positive.

Consider the difference matrix

$$
M_{q, 0}(\theta)-M_{q, \frac{1}{2}}(\theta)=\left(\begin{array}{ll}
2|c(\theta)| \\
2|c(\theta)| &
\end{array}\right)
$$

whose eigenvalues we denote by $\left\{E_{i}(\theta)\right\}_{i=1}^{q}$, namely,

$$
E_{1}(\theta)=-2|c(\theta)|<0=E_{2}(\theta)=\cdots=E_{q-1}(\theta)=0<2|c(\theta)|=E_{q}(\theta) .
$$

Recall the following Lidskii inequality and dual Lidskii inequality: Let $M_{j}$, $j=1,2$ be $n \times n$ self-adjoint matrices, let $E_{1}\left(M_{j}\right) \leq E_{2}\left(M_{j}\right) \leq \cdots \leq$ $E_{n}\left(M_{j}\right)$ be the eigenvalues of $M_{j}$. Then, for the eigenvalues of the sum of the two matrices, we have 


$$
\left\{\begin{array}{l}
\sum_{l=1}^{k} E_{i_{l}}\left(M_{1}+M_{2}\right) \leq \sum_{r=1}^{k} E_{i_{r}}\left(M_{1}\right)+\sum_{s=n-k+1}^{n} E_{s}\left(M_{2}\right) \\
\sum_{l=1}^{k} E_{i_{l}}\left(M_{1}+M_{2}\right) \geq \sum_{r=1}^{k} E_{i_{r}}\left(M_{1}\right)+\sum_{s=1}^{k} E_{s}\left(M_{2}\right)
\end{array}\right.
$$

for any $1 \leq i_{1}<\cdots<i_{k} \leq n$.

By Lidskii inequalities (6.22), we have

$$
\begin{aligned}
\sum_{k=1}^{\left[\frac{q+1}{2}\right]} \lambda_{q-2 k+2}(\theta) & \leq \sum_{k=1}^{\left[\frac{q+1}{2}\right]} \tilde{\lambda}_{q-2 k+2}(\theta)+\sum_{k=1}^{\left[\frac{q+1}{2}\right]} E_{q-k+1}(\theta) \\
& =\sum_{k=1}^{\left[\frac{q+1}{2}\right]} \tilde{\lambda}_{q-2 k+2}(\theta)+2|c(\theta)|
\end{aligned}
$$

and

$$
\begin{aligned}
\sum_{k=1}^{\left[\frac{q-1}{2}\right]} \lambda_{q-2 k+1}(\theta) & \geq \sum_{k=1}^{\left[\frac{q-1}{2}\right]} \tilde{\lambda}_{q-2 k+1}(\theta)+\sum_{k=1}^{\left[\frac{q-1}{2}\right]} E_{k}(\theta) \\
& =\sum_{k=1}^{\left[\frac{q-1}{2}\right]} \tilde{\lambda}_{q-2 k+1}(\theta)-2|c(\theta)| .
\end{aligned}
$$

Hence combining (6.21) with (6.23) (6.24), we get,

$$
\left|\Sigma_{\frac{2 \pi p}{q}, \theta}\right| \leq 4|c(\theta)| .
$$

\subsection{Proof of Lemma 6.1 for even $q$}

For sets/functions that depend on $\theta$, we will sometimes substitute $\theta$ in the notation with $A \subseteq \mathbb{T}_{1}$, if corresponding sets/functions are constant on $A$.

Since $q$ is even, a simple computation shows

$$
\left\{\begin{array}{l}
\max _{\mathbb{T}_{1}} L_{q}(\theta)=L_{q}\left(\frac{6 \mathbb{Z}+1}{6 q}\right)=L_{q}\left(\frac{6 \mathbb{Z}+5}{6 q}\right)=3, \\
\min _{\mathbb{T}_{1}} l_{q}(\theta)=l_{q}\left(\frac{2 \mathbb{Z}+1}{2 q}\right)=-6 .
\end{array}\right.
$$


A simple computation also shows $l_{q}\left(\frac{6 \mathbb{Z}+1}{6 q}\right)=-1$ and $L_{q}\left(\frac{2 \mathbb{Z}+1}{2 q}\right)=2$. Thus we have, by (6.19),

$$
\begin{aligned}
\Sigma_{2 \pi p / q} & =\left\{\lambda:-6 \leq G_{q}(\lambda) \leq 3\right\} \\
& =\left\{\lambda:-6 \leq G_{q}(\lambda) \leq 2\right\} \bigcup\left\{\lambda:-1 \leq G_{q}(\lambda) \leq 3\right\} \\
& =\Sigma_{\frac{2 \pi p}{q}, \frac{2 \mathbb{Z}+1}{2 q}} \bigcup \Sigma_{\frac{2 \pi p}{q}, \frac{6 \mathbb{Z}+1}{6 q}}
\end{aligned}
$$

This implies

$$
\left|\Sigma_{2 \pi p / q}\right| \leq\left|\Sigma_{\frac{2 \pi p}{q}, \frac{2 \mathbb{Z}+1}{2 q}}\right|+\left|\Sigma_{\frac{2 \pi p}{q}, \frac{6 \mathbb{Z}+1}{6 q}}\right| .
$$

Now it remains to estimate $\left|\Sigma_{\frac{2 \pi p}{q}}, \frac{2 \mathbb{Z}+1}{2 q}\right|$ and $\left|\Sigma_{\frac{2 \pi p}{q}}, \frac{6 \mathbb{Z}+1}{6 q}\right|$. Since $q$ is even, let us consider $\Sigma_{\frac{2 \pi p}{q}, \frac{q+1}{2 q}}$ and $\Sigma_{\frac{2 \pi p}{q}, \frac{3 q+1}{6 q}}$.

By Lemma 6.8, we have

$$
\left\{\begin{array}{l}
\left|\Sigma_{\frac{2 \pi p}{q}, \frac{q+1}{2 q}}\right| \leq 4\left|c\left(\frac{q+1}{2 q}\right)\right|<\frac{4 \pi}{q}, \\
\left|\Sigma_{\frac{2 \pi p}{q}, \frac{3 q+1}{6 q}}\right| \leq 4\left|c\left(\frac{3 q+1}{6 q}\right)\right|<\frac{4 \pi}{3 q} .
\end{array}\right.
$$

Hence putting (6.26), (6.27) together, we have

$$
\left|\Sigma_{\frac{2 \pi p}{q}}\right|<\frac{16 \pi}{3 q}
$$

\subsection{Proof of Lemma 6.1 for odd $q$}

Since the proof for odd $q$ is very similar to that for even $q$, we only sketch the steps here.

For odd $q$, similar to (6.26), we have

$$
\left|\Sigma_{\frac{2 \pi p}{q}}\right| \leq\left|\Sigma_{\frac{2 \pi p}{q}, \frac{3 \mathbb{Z}+1}{3 q}}\right|+\left|\Sigma_{\frac{2 \pi p}{q}, \frac{\mathbb{Z}}{q}}\right| .
$$

By Lemma 6.8, we have

$$
\left\{\begin{array}{l}
\left|\Sigma_{\frac{2 \pi p}{q}, \frac{3 q-1}{6 q}}\right| \leq 4\left|c\left(\frac{3 q-1}{6 q}\right)\right|<\frac{4 \pi}{3 q} \\
\left|\Sigma_{\frac{2 \pi p}{q}, \frac{q-1}{2 q}}\right| \leq 4\left|c\left(\frac{q-1}{2 q}\right)\right|<\frac{4 \pi}{q} .
\end{array}\right.
$$


Hence putting (6.29), (6.30) together, we have

$$
\left|\Sigma_{\frac{2 \pi p}{q}}\right|<\frac{16 \pi}{3 q} .
$$

This proves the claimed result.

\section{Proof of Lemma 4.1}

Lemma 4.1 is the reduction from $\Lambda^{B}$ to the tight-binding model $Q_{\Lambda}$. We now present its proof below.

Using ideas from $[11,46]$, we can express the resolvent of the operator $\Lambda^{B}$ (3.14) by Krein's resolvent formula in terms of the resolvent of the Dirichlet Hamiltonian and the resolvent of $Q_{\Lambda}$.

For this we need to introduce a few concepts first. The $l^{2}$-space on the vertices $l^{2}(\mathcal{V}(\Lambda))$ carries the inner product

$$
\langle f, g\rangle:=\sum_{\boldsymbol{v} \in \mathcal{V}(\Lambda)} 3 f(\boldsymbol{v}) \overline{g(\boldsymbol{v})}
$$

where the factor three accounts for the number of incoming or outgoing edges at each vertex.

A convenient method from classical extension theory required to state Krein's resolvent formula, and thus to link the magnetic Schrödinger operator $H^{B}$ with an effective Hamiltonian, is the concept of boundary triples.

Definition 7.1 Let $\mathcal{T}: D(\mathcal{T}) \subset \mathscr{H} \rightarrow \mathscr{H}$ be a closed linear operator on the Hilbert space $\mathscr{H}$, then the triple $\left(\pi, \pi^{\prime}, \mathscr{H}^{\prime}\right)$, with $\mathscr{H}^{\prime}$ being another Hilbert space and $\pi, \pi^{\prime}: D(\mathcal{T}) \rightarrow \mathscr{H}^{\prime}$, is a boundary triple for $\mathcal{T}$, if

- Green's identity holds on $D(\mathcal{T})$, i.e. for all $\psi, \varphi \in D(\mathcal{T})$

$$
\langle\psi, \mathcal{T} \varphi\rangle_{\mathscr{H}}-\langle\mathcal{T} \psi, \varphi\rangle_{\mathscr{H}}=\left\langle\pi \psi, \pi^{\prime} \varphi\right\rangle_{\mathscr{H}^{\prime}}-\left\langle\pi^{\prime} \psi, \pi \varphi\right\rangle_{\mathscr{H}^{\prime}}
$$

- $\operatorname{ker}\left(\pi, \pi^{\prime}\right)$ is dense in $\mathscr{H}$.

- $\left(\pi, \pi^{\prime}\right): D(\mathcal{T}) \rightarrow \mathscr{H}^{\prime} \oplus \mathscr{H}^{\prime}$ is a linear surjection.

The following lemma applies this concept to our setting.

Lemma 7.2 The operator $\mathcal{T}^{B}: D\left(\mathcal{T}^{B}\right) \subset L^{2}(\mathcal{E}(\Lambda)) \rightarrow L^{2}(\mathcal{E}(\Lambda))$ acting as the maximal Schrödinger operator (2.11) on every edge with domain

$$
D\left(\mathcal{T}^{B}\right):=\left\{\psi \in \mathcal{H}^{2}(\mathcal{E}(\Lambda)): \text { any } \vec{e}_{1}, \vec{e}_{2} \in \mathcal{E}_{\boldsymbol{v}}(\Lambda) \text { such that } i\left(\vec{e}_{1}\right)=i\left(\vec{e}_{2}\right)=\boldsymbol{v}\right. \text { satisfy }
$$




$$
\left.\begin{array}{l}
\psi_{\vec{e}_{1}}(\boldsymbol{v})=\psi_{\vec{e}_{2}}(\boldsymbol{v}) \text { and if } t\left(\vec{e}_{1}\right)=t\left(\vec{e}_{2}\right)=\boldsymbol{v}, \\
\text { then } e^{i \tilde{\beta}_{\vec{e}_{1}}} \psi_{\vec{e}_{1}}(\boldsymbol{v})=e^{i \tilde{\beta}_{\vec{e}_{2}}} \psi_{\vec{e}_{2}}(\boldsymbol{v})
\end{array}\right\}
$$

is closed. The maps $\pi, \pi^{\prime}$ on $D\left(\mathcal{T}^{B}\right)$ defined by

$$
\begin{aligned}
\pi(\psi)(\boldsymbol{v}) & :=\frac{1}{3}\left(\sum_{i(\vec{e})=\boldsymbol{v}} \psi_{\vec{e}}(\boldsymbol{v})+\sum_{t(\vec{e})=\boldsymbol{v}} e^{i \tilde{\beta}_{\vec{e}}} \psi_{\vec{e}}(\boldsymbol{v})\right) \\
\pi^{\prime}(\psi)(\boldsymbol{v}) & :=\frac{1}{3}\left(\sum_{i(\vec{e})=\boldsymbol{v}} \psi_{\vec{e}}^{\prime}(\boldsymbol{v})-\sum_{t(\vec{e})=\boldsymbol{v}} e^{i \tilde{\beta}_{\vec{e}}} \psi_{\vec{e}}^{\prime}(\boldsymbol{v})\right)
\end{aligned}
$$

form together with $\mathscr{H}^{\prime}:=l^{2}(\mathcal{V}(\Lambda))$ a boundary triple associated to $\mathcal{T}^{B}$.

Proof The proof follows the same strategy as in [46]. The operator $\mathcal{T}^{B}$ is closed iff its domain is a closed subspace (with respect to the graph norm) of the domain of some closed extension of $\mathcal{T}^{B}$. Such a closed extension is given by $\bigoplus_{\vec{e} \in \mathcal{E}(\Lambda)} \mathcal{H}_{\vec{e}}^{2}$ on $\mathcal{H}^{2}(\mathcal{E}(\Lambda))$. To see that $D\left(\mathcal{T}^{B}\right)$ is a closed subspace of $\mathcal{H}^{2}(\mathcal{E}(\Lambda)$ ), observe that in terms of continuous functionals

$$
\begin{aligned}
& l_{\vec{e}_{i}, \vec{e}_{j}}: \mathcal{H}^{2}(\mathcal{E}(\Lambda)) \rightarrow \mathbb{C}, \quad l_{\vec{e}_{i}, \vec{e}_{j}}(\psi)=\psi_{\vec{e}_{i}}\left(i\left(\vec{e}_{i}\right)\right)-\psi_{\vec{e}_{j}}\left(i\left(\vec{e}_{j}\right)\right) \\
& k_{\vec{e}_{i}, \vec{e}_{j}}: \mathcal{H}^{2}(\mathcal{E}(\Lambda)) \rightarrow \mathbb{C}, \quad k_{\vec{e}_{i}, \vec{e}_{j}}(\psi)=e^{i \tilde{\beta}_{\vec{e}_{i}}} \psi_{\vec{e}_{i}}\left(t\left(\vec{e}_{i}\right)\right)-e^{i \tilde{\beta}_{\vec{e}_{j}}} \psi_{\vec{e}_{j}}\left(t\left(\vec{e}_{j}\right)\right)
\end{aligned}
$$

we obtain

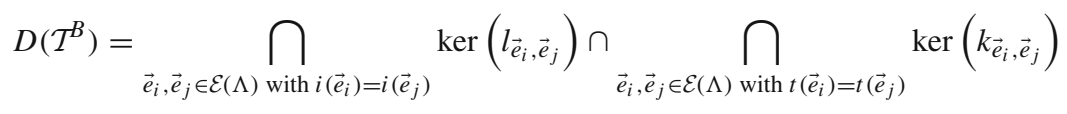

which proves closedness of $\mathcal{T}^{B}$. Green's identity follows directly from integration by parts on the level of edges. The denseness of $\operatorname{ker}\left(\pi, \pi^{\prime}\right)$ is obvious since this space contains $\bigoplus_{\vec{e} \in \mathcal{E}(\Lambda)} C_{c}^{\infty}(\vec{e})$. To show surjectivity, it suffices to consider a single edge. On those however, the property can be established by explicit constructions as in Lemma 2 in [46].

Any boundary triple for $\mathcal{T}$ as in Definition 7.1 and any self-adjoint relation $A \subseteq \mathscr{H}^{\prime} \oplus \mathscr{H}^{\prime}$ gives rise [50] to a self-adjoint restriction $\mathcal{T}_{A}$ of $\mathcal{T}$ with domain

$$
D\left(\mathcal{T}_{A}\right)=\left\{\psi \in D(\mathcal{T}):\left(\pi(\psi), \pi^{\prime}(\psi)\right) \in A\right\} .
$$

The restriction of $\mathcal{T}^{B}$ satisfying Dirichlet type boundary conditions on every edge is obtained by selecting $A_{1}:=\{0\} \oplus l^{2}(\mathcal{V}(\Lambda))$ and coincides with $H^{D}$ 
(2.14). The operator $\Lambda^{B}$ (3.14) is recovered from $\mathcal{T}^{B}$ by picking the relation $A_{2}:=l^{2}(\mathcal{V}(\Lambda)) \oplus\{0\}$.

Definition 7.3 Given the boundary triple for $\mathcal{T}^{B}$ as above, the gamma-field $\gamma: \rho\left(H^{D}\right) \rightarrow \mathcal{L}\left(l^{2}(\mathcal{V}(\Lambda)), L^{2}(\mathcal{E}(\Lambda))\right)$ is given by $\gamma(\lambda):=\left(\left.\pi\right|_{\operatorname{ker}\left(\mathcal{T}^{B}-\lambda\right)}\right)^{-1}$ and the Weyl function $M(\cdot, \Phi): \rho\left(H^{D}\right) \rightarrow \mathcal{L}\left(l^{2}(\mathcal{V}(\Lambda))\right)$ is defined as $M(\lambda, \Phi):=\pi^{\prime} \gamma(\lambda)$.

A computation shows that those maps are well-defined.

Lemma 7.4 For the operator $\mathcal{T}^{B}$, the gamma-field $\gamma$ and Weyl function $M$ can be explicitly written in terms of the solutions $s_{\lambda}, c_{\lambda}$ (2.35) on an arbitrary edge $\vec{e} \in \mathcal{E}(\Lambda)$ for $\lambda \in \rho\left(H^{D}\right)$ and $z \in l^{2}(\mathcal{V}(\Lambda))$ by

$$
(\gamma(\lambda) z) \vec{e}(\boldsymbol{x})=\frac{\left(s_{\lambda}(1) c_{\lambda, \vec{e}}(\boldsymbol{x})-s_{\lambda, \vec{e}}(\boldsymbol{x}) c_{\lambda}(1)\right) z(i(\vec{e}))+e^{-i \tilde{\beta}_{\vec{e}}} s_{\lambda, \vec{e}}(\boldsymbol{x}) z(t(\vec{e}))}{s_{\lambda}(1)}
$$

and

$$
M(\lambda, \Phi)=\frac{K_{\Lambda}(\Phi)-\Delta(\lambda)}{s_{\lambda}(1)}
$$

where

$$
\left(K_{\Lambda}(\Phi) z\right)(\boldsymbol{v}):=\frac{1}{3}\left(\sum_{\vec{e}: i(\vec{e})=\boldsymbol{v}} e^{-i \tilde{\beta}_{\vec{e}}} z(t(\vec{e}))+\sum_{\vec{e}: t(\vec{e})=v} e^{i \tilde{\beta}_{\vec{e}}} z(i(\vec{e}))\right)
$$

defines an operator in $\mathcal{L}\left(l^{2}(\mathcal{V}(\Lambda))\right)$ with $\left\|K_{\Lambda}(\Phi)\right\| \leq 1$.

Proof For $\lambda \in \rho\left(H^{D}\right)$ and $z \in l^{2}(\mathcal{V}(\Lambda))$ we define for $\vec{e} \in \mathcal{E}(\Lambda)$ arbitrary

$$
\psi_{\vec{e}}:=(\gamma(\lambda) z)_{\vec{e}}=\left(\left(\left.\pi\right|_{\operatorname{ker}\left(\mathcal{T}^{B}-\lambda\right)}\right)^{-1} z\right)_{\vec{e}}
$$

with $\psi:=\left(\psi_{\vec{e}}\right)$. In particular, $\psi_{\vec{e}}$ is the solution to $-\psi_{\vec{e}}^{\prime \prime}+V_{\vec{e}} \psi_{\vec{e}}=\lambda \psi_{\vec{e}}$ with the following boundary condition: $\psi_{\vec{e}}(i(\vec{e}))=z(i(\vec{e}))$ and $\psi_{\vec{e}}(t(\vec{e}))=$ $e^{-i \tilde{\beta}_{\vec{e}}} z(t(\vec{e}))$. The representation (7.8) is then an immediate consequence of (2.28).

The expression for the Weyl function on the other hand, follows from the Dirichlet-to-Neuman map (2.29).

$$
\begin{aligned}
(M(\lambda, \Phi) z)(\boldsymbol{v}) & =\left(\pi^{\prime} \gamma(\lambda) z\right)(\boldsymbol{v}) \\
& =\frac{1}{3}\left(\sum_{\vec{e}: i(\vec{e})=\boldsymbol{v}} \psi_{\vec{e}}^{\prime}(\boldsymbol{v})-\sum_{\vec{e}: t(\vec{e})=\boldsymbol{v}} e^{i \tilde{\beta}_{\vec{e}}} \psi_{\vec{e}}^{\prime}(\boldsymbol{v})\right)
\end{aligned}
$$




$$
\begin{aligned}
& =\frac{\left(K_{\Lambda}(\Phi) z\right)(\boldsymbol{v})}{s_{\lambda}(1)}-(\underbrace{\frac{c_{\lambda}(1)}{s_{\lambda}(1)} \delta_{\boldsymbol{v} \in i(\mathcal{V}(\Lambda))} z(\boldsymbol{v})+\frac{s_{\lambda}^{\prime}(1)}{s_{\lambda}(1)} \delta_{\boldsymbol{v} \in t(\mathcal{V}(\Lambda))} z(\boldsymbol{v})}_{=\frac{s_{\lambda}^{\prime}(1)}{s_{\lambda}(1)} z(\boldsymbol{v})}) \\
& =\frac{\left(K_{\Lambda}(\Phi) z-s_{\lambda}^{\prime}(1) z\right)(\boldsymbol{v})}{s_{\lambda}(1)},
\end{aligned}
$$

here we used (2.31). The formula (7.9) then follows from (7.12) and (2.37). Since $i(\Lambda) \cap t(\Lambda)=\emptyset$, we have $\left\|K_{\Lambda}(\Phi)\right\| \leq 1$.

The resolvents of $H^{D}=\mathcal{T}_{A_{1}}^{B}$ and $\Lambda^{B}=\mathcal{T}_{A_{2}}^{B}$ are then related by Krein's resolvent formula [50, Theorem 14.18] and a unitary equivalence between $\Lambda^{B}$ and $K_{\Lambda}(\Phi)$ away from the Dirichlet spectrum holds $[47,48]$

Theorem 9 Let $\left(l^{2}(\mathcal{V}(\Lambda)), \pi, \pi^{\prime}\right)$ be the boundary triple for $\mathcal{T}^{B}$ and $\gamma, M$ as above, then for $\lambda \in \rho\left(H^{D}\right) \cap \rho\left(\Lambda^{B}\right)$ there is also a bounded inverse of $M(\lambda, \Phi)$ and

$$
\left(\Lambda^{B}-\lambda\right)^{-1}-\left(H^{D}-\lambda\right)^{-1}=-\gamma(\lambda) M(\lambda, \Phi)^{-1} \gamma(\bar{\lambda})^{*}
$$

In particular, $\sigma\left(\Lambda^{B}\right) \backslash \sigma\left(H^{D}\right)=\left\{\lambda \in \mathbb{R} \cap \rho\left(H^{D}\right): 0 \in \sigma(M(\lambda, \Phi))\right\}$ and for intervals $J \subset \mathbb{R} \backslash \sigma\left(H^{D}\right)$

$$
\Delta\left(\Lambda^{B} \mathbb{1}_{J}\left(\Lambda^{B}\right)\right)=U\left[K_{\Lambda}(\Phi) \mathbb{1}_{\Delta(J)}\left(K_{\Lambda}(\Phi)\right)\right] U^{*}
$$

with unitary operator $U: \operatorname{ran}\left(K_{\Lambda}(\Phi) \mathbb{1}_{\Delta(J)}\left(K_{\Lambda}(\Phi)\right)\right) \rightarrow \operatorname{ran}\left(\Lambda^{B} \mathbb{1}_{J}\left(\Lambda^{B}\right)\right)$ given by

$$
U=\int_{J} \sqrt{\frac{\partial_{\lambda} s_{\lambda}(1)}{\Delta^{\prime}(\lambda)}} \gamma(\lambda) d \mathcal{E}_{K_{\Lambda}(\Phi)}(\Delta(\lambda))
$$

and $\mathcal{E}_{K_{\Lambda}(\Phi)}$ is the spectral measure of the self-adjoint operator $K_{\Lambda}(\Phi)$.

Since all vertices are integer translates of either of the two vertices $\boldsymbol{r}_{0}, \boldsymbol{r}_{1} \in$ $W_{\Lambda}$ by basis vectors $\vec{b}_{1}, \vec{b}_{2}$, we conclude that $l^{2}(\mathcal{V}(\Lambda)) \simeq l^{2}\left(\mathbb{Z}^{2} ; \mathbb{C}^{2}\right)$. Our next Lemma shows $K_{\Lambda}(\Phi)$ and $Q_{\Lambda}(\Phi)$ are unitary equivalent under this identification.

Lemma 7.5 $K_{\Lambda}(\Phi)$ is unitary equivalent to operator $Q_{\Lambda}(\Phi)$.

Proof By (3.16), (7.10),

$$
\left\{\begin{array}{l}
\left(K_{\Lambda}(\Phi) z\right)\left(\gamma_{1}, \gamma_{2}, \boldsymbol{r}_{0}\right)=\frac{1}{3}\left(z\left(\gamma_{1}, \gamma_{2}, \boldsymbol{r}_{1}\right)+z\left(\gamma_{1}-1, \gamma_{2}, \boldsymbol{r}_{1}\right)+e^{-i \Phi \gamma_{1}} z\left(\gamma_{1}, \gamma_{2}-1, \boldsymbol{r}_{1}\right)\right), \\
\left(K_{\Lambda}(\Phi) z\right)\left(\gamma_{1}, \gamma_{2}, \boldsymbol{r}_{1}\right)=\frac{1}{3}\left(z\left(\gamma_{1}, \gamma_{2}, \boldsymbol{r}_{0}\right)+z\left(\gamma_{1}+1, \gamma_{2}, \boldsymbol{r}_{0}\right)+e^{i \Phi \gamma_{1}} z\left(\gamma_{1}, \gamma_{2}+1, \boldsymbol{r}_{0}\right)\right) .
\end{array}\right.
$$


In order to transform $K_{\Lambda}$ to $Q_{\Lambda}$ we use the unitary identification $W$ : $l^{2}(\mathcal{V}(\Lambda)) \rightarrow l^{2}\left(\mathbb{Z}^{2}, \mathbb{C}^{2}\right)$

$$
(W z)_{\gamma_{1}, \gamma_{2}}:=\left(z\left(\gamma_{1}, \gamma_{2}, \boldsymbol{r}_{0}\right), z\left(\gamma_{1}, \gamma_{2}, \boldsymbol{r}_{1}\right)\right)^{T} \text {. }
$$

This way, $Q_{\Lambda}(\Phi)=W K_{\Lambda}(\Phi) W^{*}$.

Remark 6 In terms of $a \in l^{2}\left(\mathbb{Z}^{2}, \mathbb{C}^{2}\right)$ defined as

$$
\begin{aligned}
a_{(0,0)} & :=\frac{1}{3}\left(\begin{array}{ll}
0 & 1 \\
1 & 0
\end{array}\right), a_{(0,1)}:=\frac{1}{3}\left(\begin{array}{ll}
0 & 1 \\
0 & 0
\end{array}\right) \\
a_{(1,0)} & :=\frac{1}{3}\left(\begin{array}{ll}
0 & 1 \\
0 & 0
\end{array}\right), a_{(0,-1)}:=\frac{1}{3}\left(\begin{array}{ll}
0 & 0 \\
1 & 0
\end{array}\right) \\
a_{(-1,0)} & :=\frac{1}{3}\left(\begin{array}{ll}
0 & 0 \\
1 & 0
\end{array}\right), \text { and } a_{\gamma}:=0 \text { for other } \gamma \in \mathbb{Z}^{2},
\end{aligned}
$$

we can express (4.1) in the compact form

$$
Q_{\Lambda}(\Phi)=\sum_{\boldsymbol{\gamma} \in \mathbb{Z}^{2} ;|\boldsymbol{\gamma}| \leq 1} a_{\boldsymbol{\gamma}}\left(\tau_{0}\right)^{\gamma_{1}}\left(\tau_{1}\right)^{\gamma_{2}}
$$

where $|\boldsymbol{\gamma}|:=\left|\gamma_{1}\right|+\left|\gamma_{2}\right|$. This operator has already been studied, in different contexts, for rational flux quanta in $[4,28,40]$.

Finally, we point out that Lemma 4.1 follows from a combination of Theorem 9 and Lemmas 7.4 and 7.5.

\section{Spectral analysis}

This section is devoted to complete spectral analysis of $H^{B}$.

In view of Lemmas 4.1 and 5.1, an important technical fact is:

Lemma 8.1 The operator norm of $Q_{\Lambda}(\Phi)$ for non-trivial flux quanta $\Phi \notin$ $2 \pi \mathbb{Z}$ is strictly less than 1.

Indeed, then, away from the Dirichlet spectrum $\sigma\left(H^{D}\right)$, which are located on the edges of the Hill bands (2.40), we have the following characterization of $\sigma\left(H^{B}\right)$. Let $B_{n}$ and $\Delta$ be defined as in Sect. 2.2.3.

Lemma 8.2 For the magnetic Schrödinger operator $H^{B}$, the following properties hold.

(1) The level of the Dirac points $\left.\Delta\right|_{\operatorname{int}\left(B_{n}\right)} ^{-1}(0)$ always belongs to the spectrum of $H^{B}$, i.e. $\left.0 \in \Delta\right|_{\operatorname{int}\left(B_{n}\right)}\left(\sigma\left(H^{B}\right)\right)$. 
(2) $\left.\lambda \in \Delta\right|_{\operatorname{int}\left(B_{n}\right)}\left(\sigma\left(H^{B}\right)\right)$ iff $-\left.\lambda \in \Delta\right|_{\operatorname{int}\left(B_{n}\right)}\left(\sigma\left(H^{B}\right)\right)$. Consequently, the property $\Delta^{\prime}\left(\left.\Delta\right|_{\text {int }\left(B_{n}\right)} ^{-1}(0)\right) \neq 0$ implies that locally with respect to the Dirac points, the spectrum of $H^{B}$ is symmetric.

(3) $H^{B}$ has no point spectrum away from $\sigma\left(H^{D}\right)$.

(4) For non-trivial flux $\Phi \notin 2 \pi \mathbb{Z}, H^{B}$ has purely continuous spectrum bounded away from $\sigma\left(H^{D}\right)$.

In this paper, we only show the energy $\left.\Delta\right|_{\operatorname{int}\left(B_{n}\right)} ^{-1}(0)$ belongs to the spectrum of $H^{B}$. In [12] the first two authors show that not only this energy belongs to the spectrum, but also that Dirac cones actually form around this energy for any $\Phi \in 2 \pi \mathbb{Q}$.

Combining Lemma 8.2 with Lemma 4.4, we get

Lemma 8.3 For generic $\Phi, \operatorname{dim}_{H}\left(\sigma^{\Phi}\right) \leq \frac{1}{2}$.

\section{Proof of Lemma 8.3}

Lemmas 4.4 and 8.1, which implies that $\left.\Delta^{-1}\right|_{B_{n}}$ is Lipschitz on $\sigma\left(Q_{\Lambda}(\Phi)\right)$ for $\Phi \notin 2 \pi \mathbb{Z}$, show that for generic $\Phi$,

$$
\operatorname{dim}_{H}\left(\left.\Delta\right|_{\operatorname{int}\left(B_{n}\right)} ^{-1}\left(\sigma\left(Q_{\Lambda}(\Phi)\right)\right) \leq \frac{1}{2}\right.
$$

Hence since

$$
\sigma^{\Phi}=\sigma\left(H^{D}\right) \bigcup\left(\left.\cup_{n \in \mathbb{N}} \Delta\right|_{\operatorname{int}\left(B_{n}\right)} ^{-1}\left(\sigma\left(Q_{\Lambda}(\Phi)\right)\right)\right.
$$

we have

$\operatorname{dim}_{H}\left(\sigma^{\Phi}\right) \leq \sup \left\{\operatorname{dim}_{H}\left(\sigma\left(H^{D}\right)\right), \sup _{n \in \mathbb{N}} \operatorname{dim}_{H}\left(\left.\Delta\right|_{\text {int }\left(B_{n}\right)} ^{-1}\left(\sigma\left(Q_{\Lambda}(\Phi)\right)\right)\right\} \leq \frac{1}{2}\right.$

This proves Lemma 8.3.

\section{Proof of Lemma 8.2}

(1), (2) follow from a quick combination of Lemmas 4.1 and 5.1, and (3) follows from Part (2) of Lemma 5.2. (4) is a corollary of Lemma 8.1 and (3). 


\section{Proof of Lemma 8.1}

Without loss of generality $\Phi \in(0,2 \pi)$. By (5.3), it suffices to show $\left\|H_{\Phi, \theta}\right\|<$ $c_{\Phi}<6$ for some constant $c_{\Phi}$ independent of $\theta \in \mathbb{T}_{1}$. Let us take $\varphi \in \ell^{2}(\mathbb{Z})$ with $\|\varphi\|_{\ell^{2}(\mathbb{Z})}=1$. Consider

$$
\begin{aligned}
\left(H_{\Phi, \theta} \varphi\right)_{n}= & =\underbrace{c\left(\theta+n \frac{\Phi}{2 \pi}\right) \varphi_{n+1}}_{=:\left(h_{1} \varphi\right)_{n}}+\underbrace{c \overline{\left(\theta+(n-1) \frac{\Phi}{2 \pi}\right)} \varphi_{n-1}}_{=:\left(h_{2} \varphi\right)_{n}} \\
& +\underbrace{v\left(\theta+n \frac{\Phi}{2 \pi}\right) \varphi_{n}}_{=:\left(h_{3} \varphi\right)_{n}},
\end{aligned}
$$

in which $h_{1}, h_{2}, h_{3} \in \mathcal{L}\left(\ell^{2}(\mathbb{Z})\right)$. Hence

$$
\begin{aligned}
\left\|H_{\Phi, \theta} \varphi\right\|_{\ell^{2}(\mathbb{Z})}^{2} & \leq 3\left(\left\|h_{1} \varphi\right\|_{\ell^{2}(\mathbb{Z})}^{2}+\left\|h_{2} \varphi\right\|_{\ell^{2}(\mathbb{Z})}^{2}+\left\|h_{3} \varphi\right\|_{\ell^{2}(\mathbb{Z})}^{2}\right) \\
& \leq 3 \sup _{n \in \mathbb{Z}}\left(\left|c\left(\theta+(n-1) \frac{\Phi}{2 \pi}\right)\right|^{2}+\left|c\left(\theta+n \frac{\Phi}{2 \pi}\right)\right|^{2}+\left|v\left(\theta+n \frac{\Phi}{2 \pi}\right)\right|^{2}\right) \\
& \leq 12 \sup _{\theta \in \mathbb{T}_{1}}\left(\cos ^{2} \pi\left(\theta-\frac{\Phi}{2 \pi}\right)+\cos ^{2}(\pi \theta)+\cos ^{2}(2 \pi \theta)\right) \\
& =: c_{\Phi}^{2}<36 .
\end{aligned}
$$

In order to investigate further the Dirichlet spectrum and spectral decomposition of the continuous spectrum into absolutely and singular continuous parts, we start with constructing magnetic translations.

\subsection{Magnetic translations}

Below, let $\boldsymbol{\gamma}=\left(\gamma_{1}, \gamma_{2}\right)$ be in $\mathbb{Z}^{2}$ and $\vec{e}=\left(\tilde{\gamma}_{1}, \tilde{\gamma}_{2},[\vec{e}]\right)$ an arbitrary edge.

In general, $\Lambda^{B}$ does not commute with lattice translations $T_{\gamma}^{s t}$. Yet, there is a set of modified translations, introduced by [54], that do still commute with $\Lambda^{B}$, although they in general no longer commute with each other. We define those magnetic translations $T_{\gamma}^{B}: L^{2}(\mathcal{E}(\Lambda)) \rightarrow L^{2}(\mathcal{E}(\Lambda))$ as unitary operators given by

$$
\left(T_{\gamma}^{B} \psi\right)_{\vec{e}}:=u_{\gamma}^{B}(\vec{e})\left(T_{\gamma}^{\mathrm{st}} \psi\right)_{\vec{e}}
$$

for any $\psi:=\left(\psi_{\vec{e}}\right)_{\vec{e} \in \mathcal{E}(\Lambda)} \in L^{2}(\mathcal{E}(\Lambda))$ and $\gamma \in \mathbb{Z}^{2}$. The lattice translation $T_{\gamma}^{s t}$ is defined by $\left(T_{\gamma}^{s t} \psi\right)_{\vec{e}}(x)=\psi_{\vec{e}-\gamma_{1} \vec{b}_{1}-\gamma_{2} \vec{b}_{2}}\left(x-\gamma_{1} \vec{b}_{1}-\gamma_{2} \vec{b}_{2}\right)$ as before. The function $u_{\gamma}^{B}$ is constant on each copy of the fundamental domain, and defined as follows 
$u_{\gamma}^{B}\left(\tilde{\gamma}_{1}, \tilde{\gamma}_{2},[\vec{e}]\right)=e^{i \Phi \gamma_{1} \tilde{\gamma}_{2}}, \quad$ for $[\vec{e}]=\vec{f}, \vec{g}$ or $\vec{h}, \quad \gamma=\left(\gamma_{1}, \gamma_{2}\right) \in \mathbb{Z}^{2}$.

By the definition (8.1), (8.2), it is clear that for any $\psi \in L^{2}(\mathcal{E}(\Lambda))$,

$$
\frac{d}{d t} T_{\gamma}^{B} \psi=T_{\gamma}^{B} \frac{d}{d t} \psi \text { and } V T_{\gamma}^{B} \psi=T_{\gamma}^{B} V \psi
$$

In order to make sure $D\left(\Lambda^{B} T_{\gamma}^{B}\right)=D\left(T_{\gamma}^{B} \Lambda^{B}\right)$, it suffices to check $T_{\gamma}^{B}\left(D\left(\Lambda^{B}\right)\right)=D\left(\Lambda^{B}\right)$, which translates into

$$
\left\{\begin{array}{c}
u_{\gamma}\left(\vec{e}_{1}\right)=u_{\gamma}\left(\vec{e}_{2}\right) \text { whenever } i\left(\vec{e}_{1}\right)=i\left(\vec{e}_{2}\right) \\
\frac{e^{i \tilde{\beta}_{\vec{e}_{2}}} u_{\gamma}\left(\vec{e}_{2}\right)}{e^{i \vec{\beta}_{\vec{e}_{1}}} u_{\gamma}\left(\vec{e}_{1}\right)}=\frac{e^{i \tilde{\beta}_{\vec{e}_{2}}-\gamma_{1} \vec{b}_{1}-\gamma_{2} \vec{b}_{2}}}{e^{i \tilde{\beta}_{\vec{e}_{1}}-\gamma_{1} \vec{b}_{1}-\gamma_{2} \vec{b}_{2}}} \text { whenever } t\left(\vec{e}_{1}\right)=t\left(\vec{e}_{2}\right) .
\end{array}\right.
$$

This, by (3.16) is in turn equivalent to the following: for any $\tilde{\gamma}_{1}, \tilde{\gamma}_{2} \in \mathbb{Z}$ :

$$
\left\{\begin{array}{c}
u_{\gamma}\left(\tilde{\gamma}_{1}, \tilde{\gamma}_{2}, \vec{f}\right)=u_{\gamma}\left(\tilde{\gamma}_{1}, \tilde{\gamma}_{2}, \vec{g}\right)=u_{\gamma}\left(\tilde{\gamma}_{1}, \tilde{\gamma}_{2}, \vec{h}\right) \\
u_{\gamma}\left(\tilde{\gamma}_{1}, \tilde{\gamma}_{2}, \vec{f}\right)=u_{\gamma}\left(\tilde{\gamma}_{1}+1, \tilde{\gamma}_{2}, \vec{g}\right)=e^{-i \Phi \gamma_{1}} u_{\gamma}\left(\tilde{\gamma}_{1}, \tilde{\gamma}_{2}+1, \vec{h}\right)
\end{array}\right.
$$

The definition of $u_{\gamma}^{B}$ (8.2) clearly satisfies this requirement.

Therefore, although magnetic translations do not necessarily commute with one another, they commute with $\Lambda^{B}$

$$
T_{\boldsymbol{\gamma}}^{B} \Lambda^{B}=\Lambda^{B} T_{\boldsymbol{\gamma}}^{B}
$$

\subsection{Dirichlet spectrum}

In this subsection, we will study the energies belonging to the Dirichlet spectrum $\sigma\left(H^{D}\right)$. Lemma 8.4 below shows that $\sigma\left(H^{D}\right)$ is contained in the point spectrum of $H^{B}$, hence the only point spectrum of $H^{B}$, due to Part (3) of Lemma 8.2.

Consider a compactly supported simply closed loop, which is a path with vertices of degree 2 enclosing $q$ hexagons, see e.g. Fig. 7. Then this loop passes (proceeding in positive direction from the center of an edge $\vec{e}_{1}$ such that the first vertex we reach is $\left.t\left(\vec{e}_{1}\right)\right) n$ edges $\vec{e}_{1}, \ldots, \vec{e}_{n}$ in $\mathcal{E}(\Lambda)$, where $n$ is an even number. For a solution vanishing outside this loop, the boundary conditions imposed by (3.15) on the derivatives can be represented in a matrix equation

$$
T_{\Phi}(n) \psi^{\prime}(n)=0,
$$


where

$$
\begin{aligned}
& T_{\Phi}(n):=\left(\begin{array}{ccccccc}
e^{i \tilde{\beta}_{\vec{e}_{1}}} & e^{i \tilde{\beta}_{\vec{e}_{2}}} & 0 & 0 & 0 & \ldots & 0 \\
0 & 1 & 1 & 0 & 0 & \ldots & 0 \\
0 & 0 & e^{i \tilde{\beta}_{\vec{e}_{3}}} & e^{i \tilde{\beta}_{\vec{e}_{4}}} & 0 & \ldots & 0 \\
\vdots & \vdots & 0 & \ddots & \ddots & 0 & 0 \\
0 & 0 & 0 & 0 & 0 & e^{i \tilde{\beta}_{\vec{e}_{n-1}}} & e^{i \tilde{\beta}_{\vec{e}_{n}}} \\
1 & 0 & 0 & 0 & 0 & 0 & 1
\end{array}\right) \text { and } \\
& \psi^{\prime}(n):=\left(\begin{array}{c}
\psi_{\vec{e}_{1}}^{\prime}(1) \\
\psi_{\vec{e}_{2}}^{\prime}(1) \\
\psi_{\vec{e}_{3}}^{\prime}(1) \\
\vdots \\
\psi_{\vec{e}_{n-1}}^{\prime}(1) \\
\psi_{\vec{e}_{n}}^{\prime}(1)
\end{array}\right) \text {. }
\end{aligned}
$$

Remark 7 We observe that $T_{\Phi}(n)$ can be row-reduced to an upper triangular matrix with diagonal

$$
\begin{aligned}
& \left(e^{i \tilde{\beta}_{\vec{e}_{1}}}, 1, e^{i \tilde{\beta}_{\vec{e}_{3}}}, 1, e^{i \tilde{\beta}_{\vec{e}_{5}}}, \ldots, 1, e^{i \tilde{\beta}_{\vec{e}_{n-1}}}, 1-e^{i \sum_{j=1}^{n}(-1)^{j} \tilde{\beta}_{\vec{e}_{j}}}\right) \\
& =\left(e^{i \tilde{\beta}_{\vec{e}_{1}}}, 1, e^{i \tilde{\beta}_{\vec{e}_{3}}}, 1, e^{i \tilde{\beta}_{\vec{e}_{5}}}, \ldots, 1, e^{i \tilde{\beta}_{\vec{e}_{n-1}}}, 1-e^{ \pm i q \Phi}\right)
\end{aligned}
$$

where $q$ is the number of enclosed hexagons. Hence $\operatorname{rank}\left(T_{\Phi}(n)\right)=n$ iff $q \Phi \notin 2 \pi \mathbb{Z}$ and $\operatorname{rank}\left(T_{\Phi}(n)\right)=n-1$ otherwise.

Lemma 8.4 The Dirichlet eigenvalues $\lambda \in \sigma\left(H^{D}\right)$ are contained in the point spectrum of $H^{B}$.

Proof For $\Phi \in 2 \pi \mathbb{Z}$ the statement is known [39, Theroem 3.6], thus we focus on $\Phi \notin 2 \pi \mathbb{Z}$. By unitary equivalence, it suffices to construct an eigenfunction to $\Lambda^{B}$. We will construct an eigenfunction on two adjacent hexagons $\Gamma$ as in Fig. 6. Thus, $q=2$, the total number of edges is $m=11$, of which $n=10$ are on the outer loop. Let us denote the slicing edge by $\vec{e}$ and the edges on the outer loop by $\vec{e}_{1}, \vec{e}_{2}, \ldots, \vec{e}_{10}$ (see Fig. 6). Recall that $s_{\lambda, \vec{e}}$ is the Dirichlet eigenfunction on $\vec{e}$.

By Remark 7, for $2 \Phi \in 2 \pi \mathbb{Z}$, operator $T_{\Phi}(10)$ has a non-trivial nullspace. We could take

$$
a=\left(a_{j}\right) \in \operatorname{ker}\left(T_{\Phi}(10)\right) \backslash\{0\},
$$

and an eigenfunction $\psi$ on $\Gamma$ such that $\psi_{\vec{e}}=0$ and $\psi_{\vec{e}_{j}}=a_{j} s_{\lambda, \vec{e}_{j}}$.

If $2 \Phi \notin 2 \pi \mathbb{Z}$, we take a vector $y \in \mathbb{C}^{10}$ such that $y_{2}=-1, y_{7}=-e^{i \tilde{\beta}_{\vec{e}}}$ and $y_{j}=0$ otherwise. Since in this case $T_{\Phi}(10)$ is invertible, there exists a unique 
Fig. 6 Black arrows describe the double hexagon with slicing edge $\vec{e}$ indicated by the dashed arrow

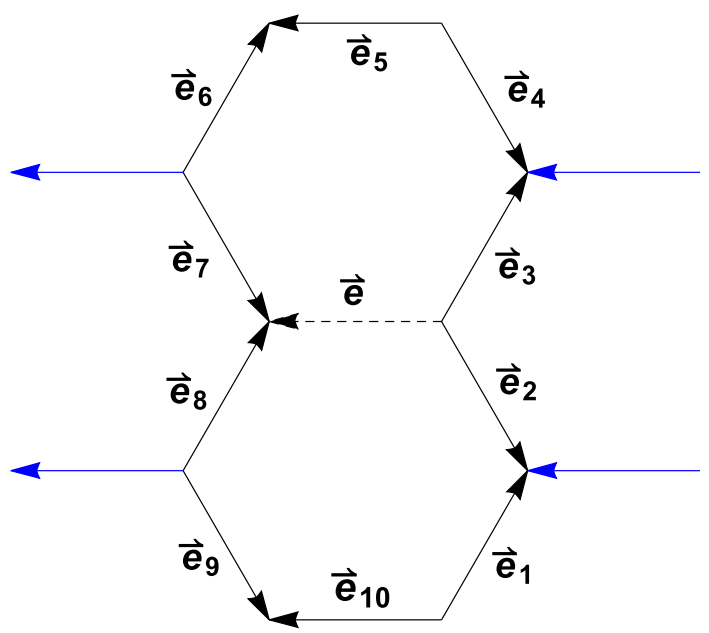

solution $a=\left(a_{j}\right)$ to the following equation:

$$
T_{\Phi}(10) a=y .
$$

Let us take $\psi$ on $\Gamma$ such that $\psi_{\vec{e}_{j}}=a_{j} s_{\lambda, \vec{e}_{j}}$ and $\psi_{\vec{e}}=s_{\lambda, \vec{e}}$, then one can easily check $\psi$ is indeed an eigenfunction on $\Gamma$.

As a corollary of Lemmas 8.1 and 8.4, we have the following:

Corollary 8.5 The spectrum of $H^{B}$ must always have open gaps for $\Phi \notin 2 \pi \mathbb{Z}$ at the edges of the Hill bands.

Remark 8 If the magnetic flux is trivial, i.e. $\Phi \in 2 \pi \mathbb{Z}$, then there do not have to be gaps. In particular, for zero potential in the non-magnetic case discussed in Theorem 6 all gaps of the absolutely continuous spectrum are closed and $\sigma_{\mathrm{ac}}\left(H^{B}\right)=[0, \infty)$.

The next lemma concerns the general feature of eigenspace of $H^{B}$. Before proceeding, let us introduce the degree of a vertex in order to distinguish different types of eigenfunctions.

Definition 8.6 An eigenfunction is said to have a vertex of degree $d$ if there is a vertex with exactly $d$ adjacent edges on which the eigenfunction does not vanish.

Lemma 8.7 For the point spectrum of $H^{B}$ it follows that

(1) Every eigenspace of $H^{B}$ is infinitely degenerated.

(2) Eigenfunctions of $H^{B}$ vanish at every vertex and are thus eigenfunctions of $H^{D}$ as well. 
(3) Eigenfunctions of $H^{B}$ with compact support cannot have vertices of degree 1. In particular, they must contain loops and the boundary edges of their support form loops as well.

Proof (1). This follows immediately using magnetic translations (8.1) and studying $\Lambda^{B}$, instead. Assume there was a finite-dimensional eigenspace of $\Lambda^{B}$. Because magnetic translations commute with $\Lambda^{B}$, they leave the eigenspaces of $\Lambda^{B}$ invariant. Magnetic translations are unitary, thus there is for any magnetic translation a normalized eigenfunction $\psi$ with eigenvalue $\lambda$ on the unit circle in $\mathbb{C}$. For $\psi$, there is a sufficiently large ball $B(0, R)$ such that

$$
\|\psi\|_{L^{2}(\mathcal{E}(\Lambda) \cap B(0, R))}>1-\varepsilon .
$$

Upon $n$-fold application of the magnetic translation, the point 0 gets translated to some point $x_{n}$ whereas the eigenfunction $\psi$ acquires only a complex phase $\lambda^{n}$. Thus, (8.10) still holds and we must also have that

$$
\|\psi\|_{L^{2}\left(\mathcal{E}(\Lambda) \cap B\left(x_{n}, R\right)\right)}>1-\varepsilon .
$$

Yet, there exists $n$ such that $B(0, R) \cap B\left(x_{n}, R\right)=\emptyset$. Therefore, (8.10) and (8.11) cannot hold at the same time for arbitrarily large $n$. This contradicts the existence of an eigenfunction to magnetic translations and thus the existence of a finite-dimensional eigenspace.

(2). If there is an eigenfunction to $H^{B}$ with eigenvalue $\lambda$ that does not vanish at a vertex, by (modified) Peierls' substitution (3.13), there is one to $\Lambda^{B}$, denoted as $\varphi$, as well. We may expand the function in local coordinates on every edge $\vec{e} \in \mathcal{E}(\Lambda)$ as $\varphi_{\vec{e}}=a_{\vec{e}} c_{\lambda, \vec{e}}+b_{\vec{e}} s_{\lambda, \vec{e}}$ according to (2.35). Recall also that the Dirichlet eigenfunction $s_{\lambda}$ is either even or odd. Thus, using (2.31) we conclude that $\left|c_{\lambda}(0)\right|=\left|c_{\lambda}(1)\right|$ and thus $\varphi$ cannot be compactly supported. In particular, $\varphi$ has the same absolute value at any vertex by boundary conditions (3.15). Due to

$$
\sum_{\vec{e} \in \mathcal{E}(\Lambda)}\left|\varphi_{\vec{e}}(i(\vec{e}))\right|^{2} \leq\|\varphi\|_{\mathcal{H}^{2}}^{2}<\infty
$$

$\varphi$ has to vanish at every vertex. Thus $\varphi$ is also an eigenfunction to $H^{D}$.

(3) clearly follows from (2) and (3.15).

\subsubsection{Dirichlet spectrum for rational flux quanta}

In this section, the flux quanta are assumed to be reduced fractions $\frac{\Phi}{2 \pi}=\frac{p}{q}$. If magnetic fields are absent, the point spectrum is spanned by hexagonal simply closed loop states, i.e. states supported on a single hexagon [39]. We will see in the following that similar statements remain true in the case of rational flux quanta and derive such a basis as well. The natural extension of 


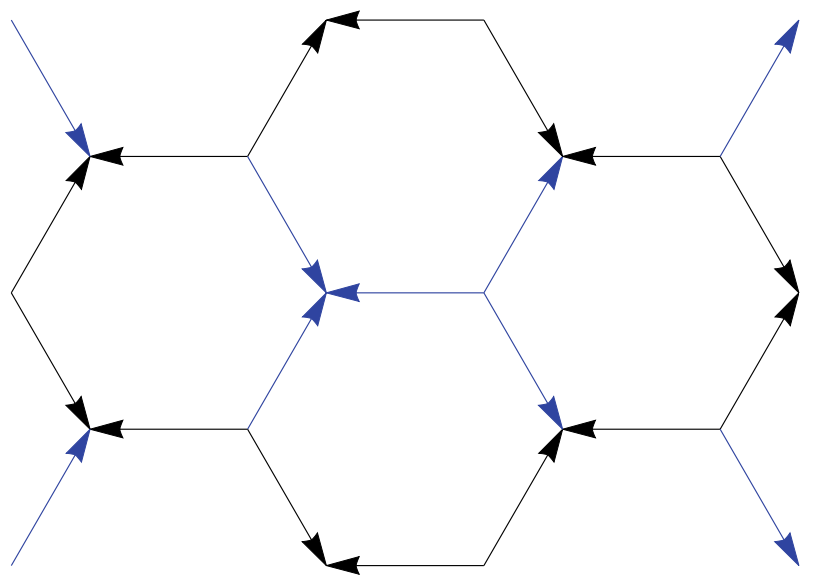

Fig. 7 Simply closed loop state supported on black arrows encloses area $\frac{4 \Phi}{B_{0}}$

loop states supported on a single hexagon, in the case of magnetic fields, are simply closed loops enclosing an area $\frac{q \Phi}{B_{0}}$ rather than just $\frac{\Phi}{B_{0}}$, see Fig. 7 .

Lemma 8.8 Any simply closed loop enclosing an area of $\frac{q \Phi}{B_{0}}$ has a unique (up to normalization) eigenfunction of $H^{B}$ supported on it.

Proof The existence of eigenfunctions on simply closed loops enclosing this flux follows directly from the non-trivial kernel of (8.7), see Remark 7. Due to $\operatorname{dim}\left(\operatorname{ker}\left(T_{\Phi}\right)\right)=1$, such eigenfunctions are also unique (up to normalization).

Lemma 8.9 The nullspaces $\operatorname{ker}\left(H^{B}-\lambda\right)$ where $\lambda \in \sigma\left(H^{D}\right)$ are generated by compactly supported eigenfunctions.

Proof Unitary equivalence allows us to work with $\Lambda^{B}$ rather than $H^{B}$. Without loss of generality, we assume that the Dirichlet eigenfunction to $\lambda$ is even. Due to Lemma 8.7, eigenfunctions of $\Lambda^{B}$ to Dirichlet eigenvalues vanish at every vertex. Thus, on every edge $\vec{e} \in \mathcal{E}(V)$, they are of the form $\varphi_{\vec{e}}=a_{\vec{e}} s_{\lambda, \vec{e}}$ for some $a_{\vec{e}}$.

Let $\varphi$ be such a function. We define the sequence $(u(v))_{v \in \mathcal{V}(\Lambda)}$ as follows

$$
\left\{\begin{array}{l}
u\left(\gamma_{1}, \gamma_{2}, \boldsymbol{r}_{0}\right):=\varphi_{\gamma_{1}, \gamma_{2}, \vec{g}}^{\prime}\left(\gamma_{1}, \gamma_{2}, \boldsymbol{r}_{0}\right) \\
u\left(\gamma_{1}, \gamma_{2}, \boldsymbol{r}_{1}\right):=\varphi_{\gamma_{1}, \gamma_{2}, \vec{f}}^{\left(\gamma_{1}, \gamma_{2}, \boldsymbol{r}_{1}\right) .}
\end{array}\right.
$$

Observe that the sequence $(u(v))$ determines the eigenfunction on every edge. Indeed, $a_{\gamma_{1}, \gamma_{2}, \vec{g}}=u\left(\gamma_{1}, \gamma_{2}, \boldsymbol{r}_{0}\right)$ and $a_{\gamma_{1}, \gamma_{2}, \vec{f}}=u\left(\gamma_{1}, \gamma_{2}, \boldsymbol{r}_{1}\right)$, since $s_{\lambda}^{\prime}(1)=$ $s_{\lambda}^{\prime}(0)$. At the same time, $a_{\gamma_{1}, \gamma_{2}, \vec{h}}$ can be determined in two different ways, one 
for each endpoint, from the boundary condition (3.15). Let us now introduce an operator $A \in \mathcal{L}\left(l^{2}(\mathcal{V}(\Lambda))\right)$ that has precisely the sequences $(u(v))$ with matching boundary conditions for $a_{\gamma_{1}, \gamma_{2}, \vec{h}}$ in its kernel. Then,

$$
\begin{aligned}
(A u)\left(\gamma_{1}, \gamma_{2}, \boldsymbol{r}_{0}\right):= & u\left(\gamma_{1}, \gamma_{2}, \boldsymbol{r}_{0}\right)+u\left(\gamma_{1}, \gamma_{2}, \boldsymbol{r}_{1}\right) \\
& -e^{2 \pi i \frac{p \gamma_{1}}{q}}\left(u\left(\gamma_{1}+1, \gamma_{2}-1, \boldsymbol{r}_{0}\right)+u\left(\gamma_{1}, \gamma_{2}-1, \boldsymbol{r}_{1}\right)\right) \text { and } \\
(A u)\left(\gamma_{1}, \gamma_{2}, \boldsymbol{r}_{1}\right):= & 0 .
\end{aligned}
$$

The operator $A$ is then a $\mathbb{Z}^{2}$-periodic finite-order difference operator. Any eigenfunction $\varphi$ satisfying $\left(\Lambda^{B}-\lambda\right) \varphi=0$ leads by standard arguments to a square-summable sequence $(u(v))$ as defined above in the nullspace of $A$. Conversely, any such element in the nullspace of $A$ uniquely defines an eigenfunction $\varphi=a_{\vec{e}} s_{\lambda, \vec{e}}$ to $\Lambda^{B}$. Theorem 8 in [36] implies then that the nullspace of $A$ is generated by sequences in $c_{00}(\mathcal{V}(\Lambda)$ ). It suffices now to observe that those compactly supported sequences also give rise to compactly supported eigenfunctions to conclude the claim.

Lemma 8.10 Let $\Phi \notin 2 \pi \mathbb{Z}$. The eigenspaces are spanned by the set of double hexagonal states, see Fig. 6.

Proof By Lemma 8.7, all eigenfunctions vanish at every vertex. Compactly supported eigenfunctions are dense in the eigenspace by the previous Lemma 8.9. Thus, it suffices, as in the non-magnetic [39] case, to show that any compactly supported eigenfunction is a linear combination of double hexagonal states. Let $\varphi$ be a compactly supported eigenfunction of $\Lambda^{B}$ to some Dirichlet eigenvalue $\lambda$. Consider an edge $\vec{d} \in \mathcal{E}(\Lambda)$ on the boundary loop of the support of $\varphi$. It exists due to (3) of Lemma 8.7. The boundary loop, which cannot be just a loop around a single hexagon, as this one does not support such eigenfunctions, necessarily encloses a double hexagon $\Gamma$, as in Fig. 6, which contains the chosen edge $\vec{d}$. Then, there is by the proof of Lemma 8.4 a state $\psi$ on $\Gamma$ so that the wavefunction $\psi_{\vec{d}}$ on $\vec{d}$ coincides with $\varphi_{\vec{d}}$. Subtracting $\psi$ from $\varphi$ leaves us with an eigenfunction to $\Lambda^{B}$ that encloses at least one single hexagon less than $\psi$. Thus, iterating this procedure shows that compactly supported eigenfunctions are spanned by double hexagonal states which implies the claim.

\subsubsection{Dirichlet spectrum for irrational flux quanta}

After proving Theorem 4 for rational flux quanta, we now prove the analogous result for irrational magnetic fluxes. We start by introducing the following definition. 
Definition 8.11 The Hilbert space $l^{2}(\mathcal{E}(\Lambda))$ is defined as

$$
l^{2}(\mathcal{E}(\Lambda)):=\left\{z: \mathcal{E}(\Lambda) \rightarrow \mathbb{C},\|z\|_{l^{2}(\mathcal{E}(\Lambda))}^{2}:=\sum_{\vec{e} \in \mathcal{E}(\Lambda)}|z(\vec{e})|^{2}<\infty\right\} .
$$

Theorem 10 The double hexagonal states generate the eigenspaces of Dirichlet spectrum of $H^{B}$ for irrational flux quanta.

We will give a proof of this theorem after a couple of auxiliary observations. For this entire discussion to follow we consider a fixed $\lambda \in \sigma\left(H^{D}\right)$.

Definition 8.12 We denote the closed $L^{2}(\mathcal{E}(\Lambda))$ subspace generated by linear combinations of all double hexagonal states on the entire graph $\Lambda$ by $D H_{\mathcal{E}(\Lambda)}(\Phi)$.

There is a countable orthonormal system of states $V(\Phi) \subset D H_{\mathcal{E}(\Lambda)}(\Phi)$ such that

$$
\overline{\operatorname{span}(V(\Phi))}=D H_{\mathcal{E}(\Lambda)}(\Phi) .
$$

We may label elements of $V(\Phi)$ by $\varphi_{\gamma}(\Phi)$ with $\boldsymbol{\gamma} \in \mathbb{Z}^{2}$. Without loss of generality, $\varphi_{\gamma}(\Phi)$ can be chosen to depend analytically on $\Phi \in(0,1)$. Every element $\varphi_{\gamma}(\Phi) \in V(\Phi)$ is due to Lemma 8.7 of the form

$$
\varphi_{\gamma}(\Phi)=\sum_{\vec{e} \in \mathcal{E}(\Lambda)} \varphi_{\gamma, \vec{e}}(\Phi) s_{\lambda, \vec{e}}
$$

because it is an element of $\operatorname{ker}\left(H^{B}-\lambda\right)$.

Now assume that the statement of Theorem 10 does not hold, this is equivalent to saying that $Z(\Phi):=\operatorname{ker}\left(H^{B}-\lambda\right) \cap D H_{\mathcal{E}(\Lambda)}(\Phi)^{\perp}$ is not the zero space, i.e. there are eigenfunctions not spanned by double hexagonal states. Our goal is to characterize $Z(\Phi)$ as the nullspace of a suitable operator we define next.

Definition 8.13 Let $A(\Phi) \in \mathcal{L}\left(l^{2}(\mathcal{E}(\Lambda))\right)$ be defined as

$$
\begin{aligned}
(A(\Phi) u)(\boldsymbol{\gamma}, \vec{f}):= & u(\boldsymbol{\gamma}, \vec{f})+u(\boldsymbol{\gamma}, \vec{g})+u(\boldsymbol{\gamma}, \vec{h}) \\
(A(\Phi) u)(\boldsymbol{\gamma}, \vec{g}):= & u\left(\gamma_{1}, \gamma_{2}-1, \vec{f}\right)+u\left(\gamma_{1}+1, \gamma_{2}-1, \vec{g}\right) \\
& +e^{-i \Phi \gamma_{1}} u\left(\gamma_{1}, \gamma_{2}, \vec{h}\right) \\
(A(\Phi) u)(\boldsymbol{\gamma}, \vec{h}):= & \left\langle u,\left.\left(\varphi_{\boldsymbol{\gamma}, \vec{e}}(\Phi)\right)\right|_{l^{2}(\mathcal{E}(\Lambda))}\right.
\end{aligned}
$$

for any $u \in l^{2}(\mathcal{E}(\Lambda))$.

Remark 9 The first two lines of this definition resemble the boundary conditions for the derivatives at outgoing/incoming vertices (3.17) and with the third line we monitor the orthogonality of $\sum_{\vec{e} \in \mathcal{E}(\Lambda)} u_{\vec{e}} s_{\lambda, \vec{e}}$ to $D H_{\mathcal{E}(\Lambda)}(\Phi)$. 
In particular, there is an isometric isomorphism $\eta \in \mathcal{L}(\operatorname{ker}(A(\Phi)), Z(\Phi))$ with

$$
\eta(u):=\sum_{\vec{e} \in \mathcal{E}(\Lambda)} \frac{u_{\vec{e}}}{\left\|s_{\lambda, \vec{e}}\right\|_{L^{2}(\vec{e})}} s_{\lambda, \vec{e}}
$$

We observe that by Lemma 8.9 and the isomorphism (8.19) the operator $A(\Phi)$ is injective for $\frac{\Phi}{2 \pi} \in \mathbb{Q} \cap(0,1)$. To prove Theorem 10 we only need the following Lemma:

Lemma 8.14 The operator $A(\Phi)$ is surjective for $\frac{\Phi}{2 \pi} \in(0,1)$. In particular, for any $(a(\vec{e})) \in l^{2}(\mathcal{E}(\Lambda))$, there exists $(u(\vec{e})) \in l^{2}(\mathcal{E}(\Lambda))$ such that $A(\Phi) u=$ $a$ and

$$
\|u\|_{l^{2}(\mathcal{E}(\Lambda))} \leq \frac{C}{\left|1-e^{-i \Phi}\right|}\|a\|_{l^{2}(\mathcal{E}(\Lambda))}
$$

holds for a universal constant $C$.

Combining Lemma 8.14 with the already established injectivity result, we have $A(\Phi)$ is continuously invertible for $\frac{\Phi}{2 \pi} \in \mathbb{Q} \cap(0,1)$ with the following control of its norm

$$
\left\|A(\Phi)^{-1}\right\| \leq \frac{C}{\left|1-e^{-i \Phi}\right|} .
$$

Now let us give the proof of Theorem 10, assuming the result of Lemma 8.14.

\section{Proof of Theorem 10}

Since $\|A(\Phi)\|$ is uniformly bounded by a constant and $\Phi \mapsto\langle x, A(\Phi) y\rangle$ is analytic for $x, y \in c_{00}(\mathcal{E}(\Lambda)), A(\Phi)$ is an analytic operator in $\Phi$. Thus for any $\frac{\tilde{\Phi}}{2 \pi} \in(0,1)$, there exists $\varepsilon_{1}(\tilde{\Phi})$ and $C(\tilde{\Phi})$ such that

$$
\|A(\Phi)-A(\tilde{\Phi})\| \leq C(\tilde{\Phi})|\Phi-\tilde{\Phi}|, \quad \text { for }|\Phi-\tilde{\Phi}|<\varepsilon_{1}(\tilde{\Phi}) .
$$

Also by $(8.21)$, for any irrational $\frac{\tilde{\Phi}}{2 \pi} \in(0,1)$ and rational $\frac{\Phi}{2 \pi}$ with $|\Phi-\tilde{\Phi}|<$ $\varepsilon_{2}(\tilde{\Phi})$, we have

$$
\left\|A(\Phi)^{-1}\right\| \leq \frac{2 C}{\left|1-e^{-i \tilde{\Phi}}\right|}
$$




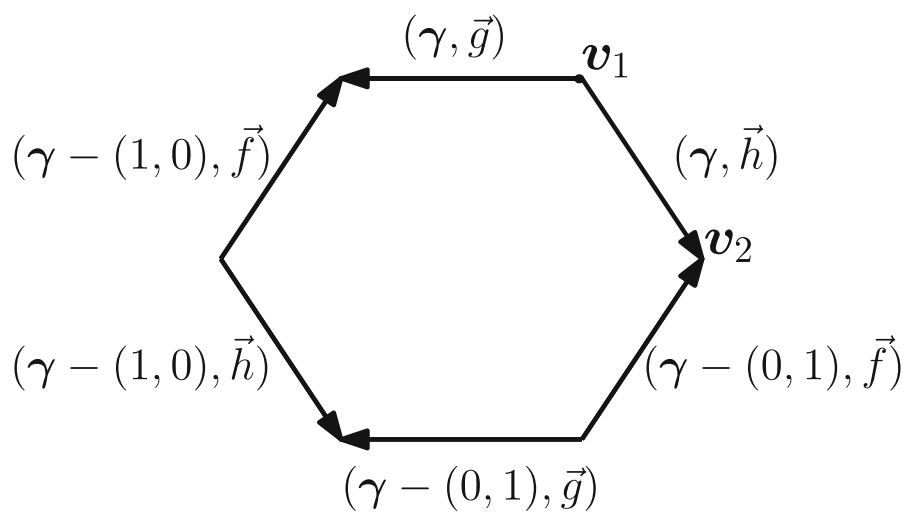

Fig. 8 Labelling of hexagon $\Gamma_{\gamma}$

Hence, taking $\frac{\Phi}{2 \pi} \in \mathbb{Q} \cap(0,1)$ that is close to $\frac{\tilde{\Phi}}{2 \pi}$ such that $|\Phi-\tilde{\Phi}|<$ $\min \left(\varepsilon_{1}(\tilde{\Phi}), \varepsilon_{2}(\tilde{\Phi}), \frac{\left|1-e^{-i \tilde{\Phi}}\right|}{2 C(\tilde{\Phi}) C}\right)$, we would get

$$
\left\|A(\Phi)^{-1}(A(\tilde{\Phi})-A(\Phi))\right\|<1 .
$$

This implies that

$$
A(\tilde{\Phi})=A(\Phi)\left(\mathrm{Id}+A(\Phi)^{-1}(A(\tilde{\Phi})-A(\Phi))\right)
$$

is invertible. Thus, we conclude that also for irrational fluxes $\operatorname{ker}(A(\Phi))=\{0\}$ and by (8.19) therefore $Z(\Phi)=\{0\}$ which shows the claim.

\section{Proof of Lemma 8.14}

We prove this Lemma by showing that there is a sufficiently sparse set of elements in $l^{2}(\mathcal{E}(\Lambda))$ that gets mapped under $A(\Phi)$ on the standard basis of $l^{2}(\mathcal{E}(\Lambda))$.

Let $\alpha_{\vec{e},(\gamma, \vec{h})}:=\varphi_{\gamma, \vec{e}}\left\|s_{\lambda, \vec{e}}\right\|_{L^{2}(\vec{e})}^{2}$. Since functions $\varphi_{\boldsymbol{\gamma}}$ satisfy the continuity conditions (3.17) and form an $L^{2}$ orthonormal system, we obtain the standard basis vectors $\delta_{\bullet,(\gamma, \vec{h})} \in l^{2}(\mathcal{E}(\Lambda))$ under $A(\Phi)$

$$
\begin{aligned}
& \left(A(\Phi) \alpha_{\bullet,(\boldsymbol{\gamma}, \vec{h})}\right)\left(\boldsymbol{\gamma}^{\prime}, \vec{f}\right):=0, \\
& \left(A(\Phi) \alpha_{\bullet,(\boldsymbol{\gamma}, \vec{h})}\right)\left(\boldsymbol{\gamma}^{\prime}, \vec{g}\right):=0, \text { and } \\
& \left(A(\Phi) \alpha_{\bullet,(\boldsymbol{\gamma}, \vec{h})}\right)\left(\boldsymbol{\gamma}^{\prime}, \vec{h}\right):=\delta_{\boldsymbol{\gamma}, \boldsymbol{\gamma}^{\prime}} .
\end{aligned}
$$


To obtain also the remaining basis vectors, let us define $L^{2}$ functions $\tilde{\psi}_{(\gamma, \vec{f})}$ and $\tilde{\psi}_{(\gamma, \vec{g})}$ supported on a single hexagon $\Gamma_{\gamma}$ as shown in Fig. 8. The indices of $\tilde{\psi}_{(\gamma,[\vec{e}])}$ are chosen to indicate the standard basis vectors $\delta_{\bullet,(\gamma,[\vec{e}])} \in l^{2}(\mathcal{E}(\Lambda))$ in the range of $A(\Phi)$ that we will construct from those functions. To define $\tilde{\psi}_{(\gamma, \vec{f})}$ and $\tilde{\psi}_{(\boldsymbol{\gamma}, \vec{g})}$, we introduce coefficients $\zeta_{\bullet,(\gamma, \vec{f})}$ and $\zeta_{\bullet,(\boldsymbol{\gamma}, \vec{g})}$ such that $\tilde{\psi}_{(\boldsymbol{\gamma}, \vec{f})}:=$ $\sum_{\vec{e} \in \mathcal{E}\left(\Gamma_{\gamma}\right)} \zeta_{\vec{e},(\gamma, \vec{f})} s_{\lambda, \vec{e}}$ and $\tilde{\psi}_{(\gamma, \vec{g})}:=\sum_{\vec{e} \in \mathcal{E}\left(\Gamma_{\gamma}\right)} \zeta_{\vec{e},(\gamma, \vec{g})} s_{\lambda, \vec{e}}$, respectively.

We do this in such a way that all continuity conditions for $\tilde{\psi}_{(\gamma, \vec{f})}$ at the vertices of $\Gamma_{\gamma}$ are satisfied up to a single one at the (initial) vertex $\boldsymbol{v}_{1}:=$ $i((\boldsymbol{\gamma}, \vec{g}))=i((\boldsymbol{\gamma}, \vec{h}))$, see Fig. 8 . We define for fixed $\vec{e}=(\boldsymbol{\gamma}, \vec{f})$

$$
\begin{aligned}
& \zeta_{(\gamma, \vec{h}), \vec{e}}:=\frac{1}{1-e^{-i \Phi}}, \zeta_{(\gamma-(0,1), \vec{f}), \vec{e}}:=\frac{-e^{-i \Phi \gamma_{1}}}{1-e^{-i \Phi}}, \zeta_{(\gamma-(0,1), \vec{g}), \vec{e}}:=\frac{e^{-i \Phi \gamma_{1}}}{1-e^{-i \Phi}}, \\
& \zeta_{(\gamma-(1,0), \vec{h}), \vec{e}}:=\frac{-e^{-i \Phi}}{1-e^{-i \Phi}}, \zeta_{(\gamma-(1,0), \vec{f}), \vec{e}}:=\frac{e^{-i \Phi}}{1-e^{-i \Phi}}, \zeta_{(\gamma, \vec{g}), \vec{e}}:=\frac{-e^{-i \Phi}}{1-e^{-i \Phi}}
\end{aligned}
$$

and all other $\zeta_{\bullet, \vec{e}}$ are taken to be zero. Since for $\tilde{\psi}_{(\gamma, \vec{f})}$ all but one continuity conditions are satisfied, we obtain for the first two components of (8.18)

$$
\left(A(\Phi) \zeta_{\bullet,(\gamma, \vec{f})}\right)\left(\boldsymbol{\gamma}^{\prime}, \vec{f}\right):=\delta_{\boldsymbol{\gamma}, \boldsymbol{\gamma}^{\prime}} \text { and }\left(A(\Phi) \zeta_{\bullet,(\boldsymbol{\gamma}, \vec{f})}\right)\left(\boldsymbol{\gamma}^{\prime}, \vec{g}\right):=0 .
$$

To ensure that we also get constant zero in the third component of (8.18), we project onto the orthogonal complement of the double hexagonal states $\psi_{(\gamma, \vec{f})}:=\widetilde{\psi}_{(\gamma, \vec{f})}-P_{D H_{\mathcal{L}(\Lambda)}(\Phi)} \widetilde{\psi}_{(\gamma, \vec{f})}$ where $P_{D H_{\mathcal{E}(\Lambda)}(\Phi)}$ is the orthogonal projection onto $D H_{\mathcal{E}(\Lambda)}(\Phi)$. Let now $\alpha_{\vec{e},(\gamma, \vec{f})}$ be such that

$$
\psi_{(\gamma, \vec{f})}=\sum_{\vec{e} \in \mathcal{E}\left(\Gamma_{\gamma}\right)} \alpha_{\vec{e},(\gamma, \vec{f})} s_{\lambda, \vec{e}}
$$

then it follows that

$$
\begin{aligned}
& \left(A(\Phi) \alpha_{\bullet,(\boldsymbol{\gamma}, \vec{f})}\right)\left(\boldsymbol{\gamma}^{\prime}, \vec{f}\right):=\delta_{\boldsymbol{\gamma}, \boldsymbol{\gamma}^{\prime}}, \\
& \left(A(\Phi) \alpha_{\bullet,(\boldsymbol{\gamma}, \vec{f})}\right)\left(\boldsymbol{\gamma}^{\prime}, \vec{g}\right):=0, \text { and } \\
& \left(A(\Phi) \alpha_{\bullet,(\boldsymbol{\gamma}, \vec{f})}\right)\left(\boldsymbol{\gamma}^{\prime}, \vec{h}\right):=0 .
\end{aligned}
$$

Similarly, we choose coefficients $\zeta_{\bullet}, \vec{e}$ with $\vec{e}=(\boldsymbol{\gamma}, \vec{g})$, such that the boundary conditions are satisfied up to the one at the (terminal) vertex $\boldsymbol{v}_{2}:=t((\boldsymbol{\gamma}, \vec{h}))=t((\boldsymbol{\gamma}-(0,1), \vec{f}))$, see Fig. 8 .

$$
\zeta_{(\gamma-(0,1), \vec{f}), \vec{e}}:=\frac{1}{1-e^{-i \Phi}}, \zeta_{(\gamma-(0,1), \vec{g}), \vec{e}}:=\frac{-1}{1-e^{-i \Phi}}, \zeta_{(\gamma-(1,0), \vec{h}), \vec{e}}
$$




$$
\begin{gathered}
:=\frac{e^{i \Phi\left(\gamma_{1}-1\right)}}{1-e^{-i \Phi}} \\
\zeta_{(\gamma-(1,0), \vec{f}), \vec{e}}:=\frac{-e^{i \Phi\left(\gamma_{1}-1\right)}}{1-e^{-i \Phi}}, \zeta_{(\gamma, \vec{g}), \vec{e}}:=\frac{e^{i \Phi\left(\gamma_{1}-1\right)}}{1-e^{-i \Phi}}, \zeta_{(\gamma, \vec{h}), \vec{e}}:=\frac{-e^{i \Phi\left(\gamma_{1}-1\right)}}{1-e^{-i \Phi}}
\end{gathered}
$$

and all other coefficients $\zeta_{\bullet}, \vec{e}$ equal to zero. Thus, we get for the first two components of (8.18)

$$
\left(A(\Phi) \zeta_{\bullet},(\boldsymbol{\gamma}, \vec{g})\right)\left(\boldsymbol{\gamma}^{\prime}, \vec{f}\right)=0 \text { and }\left(A(\Phi) \zeta_{\bullet},(\boldsymbol{\gamma}, \vec{g})\right)\left(\boldsymbol{\gamma}^{\prime}, \vec{g}\right)=\delta_{\boldsymbol{\gamma}, \boldsymbol{\gamma}^{\prime}}
$$

To ensure that we also get constant zero in the third component of (8.18), we project again on the orthogonal complement of the double hexagonal states $\psi_{(\gamma, \vec{g})}:=\widetilde{\psi}_{(\gamma, \vec{g})}-P_{D H_{\mathcal{E}(\Lambda)}(\Phi)} \widetilde{\psi}_{(\gamma, \vec{g})}$. Let now $\psi_{(\gamma, \vec{g})}=\sum_{\vec{e} \in \mathcal{E}\left(\Gamma_{\gamma}\right)} \alpha_{\vec{e},(\gamma, \vec{g})} s_{\lambda, \vec{e}}$, then

$$
\begin{aligned}
& \left(A(\Phi) \alpha_{\bullet},(\boldsymbol{\gamma}, \vec{g})\right)\left(\boldsymbol{\gamma}^{\prime}, \vec{f}\right):=0 \\
& \left(A(\Phi) \alpha_{\bullet,(\gamma, \vec{g})}\right)\left(\boldsymbol{\gamma}^{\prime}, \vec{g}\right):=\delta_{\boldsymbol{\gamma}, \boldsymbol{\gamma}^{\prime}}, \text { and } \\
& \left(A(\Phi) \alpha_{\bullet,(\gamma, \vec{g})}\right)\left(\boldsymbol{\gamma}^{\prime}, \vec{h}\right):=0 .
\end{aligned}
$$

Hence, we obtained in (8.24), (8.28), and (8.31) sequences

$$
\left\{\alpha_{\bullet,(\gamma, \vec{f})}, \alpha_{\bullet},(\boldsymbol{\gamma}, \vec{g}), \text { and } \alpha_{\bullet,(\gamma, \vec{h})} ; \boldsymbol{\gamma} \in \mathbb{Z}^{2}\right\}
$$

in $l^{2}(\mathcal{E}(\Lambda))$ that get mapped under $A(\Phi)$ onto the standard unit basis of $l^{2}(\mathcal{E}(\Lambda))$.

To conclude surjectivity of $A(\Phi)$ from this, it suffices to show that for all $(a(\vec{e})) \in l^{2}(\mathcal{E}(\Lambda))$ we can bound $u(\vec{e}):=\sum_{\vec{d} \in \mathcal{E}(\Lambda)} a(\vec{d}) \alpha_{\vec{e}, \vec{d}}$ as follows

$$
\|u\|_{l^{2}(\mathcal{E}(\Lambda))}^{2} \leq \frac{C^{2}}{\left|1-e^{-i \Phi}\right|^{2}} \sum_{\vec{e} \in \mathcal{E}(\Lambda)}|a(\vec{e})|^{2} .
$$

We then define

$$
\sigma_{\vec{e}}=\sum_{\vec{d} \in \mathcal{E}(\Lambda) ;[\vec{d}] \neq \vec{h}} a(\vec{d}) \alpha_{\vec{e}, \vec{d}} \text { and } v_{\vec{e}}=\sum_{\vec{d} \in \mathcal{E}(\Lambda) ;[\vec{d}] \neq \vec{h}} a(\vec{d}) \zeta_{\vec{e}, \vec{d}}
$$

Since $\psi_{(\gamma, \vec{f})}, \psi_{(\gamma, \vec{g})} \in D H_{\mathcal{E}(\Lambda)}(\Phi)^{\perp}$ and $\left(\varphi_{\gamma}\right)$ forms an orthonormal system in $D H_{\mathcal{E}(\Lambda)}(\Phi)$, to prove (8.33) it suffices to show

$$
\|\sigma\|_{l^{2}(\mathcal{E}(\Lambda))}^{2} \leq \frac{C^{2}}{\left|1-e^{-i \Phi}\right|^{2}} \sum_{\vec{e} \in \mathcal{E}(\Lambda) ;[\vec{e}] \neq \vec{h}}|a(\vec{e})|^{2} .
$$


Due to $\|\sigma\|_{l^{2}(\mathcal{E}(\Lambda))} \leq\|v\|_{l^{2}(\mathcal{E}(\Lambda))}+\|\sigma-v\|_{l^{2}(\mathcal{E}(\Lambda))}$ we may establish estimate (8.33) for each term on the right-hand side of the triangle inequality, individually.

For two edges $\vec{d}, \vec{e} \in \mathcal{E}(\Lambda)$ we define a function $M(\vec{d}, \vec{e}):=1$ if there are $\boldsymbol{\gamma}, \boldsymbol{\gamma}^{\prime} \in \mathbb{Z}^{2}$ and two hexagons $\Gamma_{\boldsymbol{\gamma}}, \Gamma_{\boldsymbol{\gamma}^{\prime}}$ satisfying $\Gamma_{\boldsymbol{\gamma}} \cap \Gamma_{\boldsymbol{\gamma}^{\prime}} \neq \varnothing$ such that $\vec{d} \in \Gamma_{\gamma}$ and $\vec{e} \in \Gamma_{\gamma^{\prime}}$, and $M(\vec{d}, \vec{e}):=0$ otherwise. Choosing $\tau_{1}$ such that $\sum_{\vec{d} \in \mathcal{E}(\Lambda) ;[\vec{d}] \neq \vec{h}} M(\vec{d}, \vec{e}) \leq \tau_{1}$ for any $\vec{e} \in \mathcal{E}(\Lambda)$, then

$$
\begin{aligned}
\|v\|_{l^{2}(\mathcal{E}(\Lambda))}^{2} & \leq \sum_{\vec{d}, \vec{e} \in \mathcal{E}(\Lambda) ;[\vec{d}],[\vec{e}] \neq \vec{h}}|a(\vec{d})||a(\vec{e})| \underbrace{\left\|\zeta_{\bullet}, \vec{d}\right\|_{\left.l^{2}(\mathcal{E}(\Lambda))\right)}\left\|\zeta_{\bullet}, \vec{e}\right\|_{l^{2}(\mathcal{E}(\Lambda))}}_{\leq \frac{7}{\left|1-e^{-i \Phi}\right|^{2}}} M(\vec{d}, \vec{e}) \\
& \leq \frac{6 \tau_{1}}{\left|1-e^{-i \Phi}\right|^{2}} \sum_{\vec{e} \in \mathcal{E}(\Lambda) ;[\vec{e}] \neq \vec{h}}|a(\vec{e})|^{2} .
\end{aligned}
$$

For the second term $\|\sigma-v\|_{l^{2}(\mathcal{E}(\Lambda))}$, we use that functions $\tilde{\psi}_{(\gamma,[\vec{e}])}$ with $[\vec{e}] \neq$ $[\vec{h}]$ are supported on hexagons $\Gamma$ and can therefore only overlap with finitely many linearly independent double hexagonal states. Thus, we define a function $N$ with $N(\vec{d}, \vec{e}):=1$ if $\vec{d}, \vec{e}$ belong to two hexagons $\Gamma_{\gamma}, \Gamma_{\gamma^{\prime}}$ for which there are two double hexagons $\Gamma_{1}, \Gamma_{2}$ with the property that all intersections $\Gamma_{\gamma} \cap \Gamma_{1}$, $\Gamma_{1} \cap \Gamma_{2}, \Gamma_{2} \cap \Gamma_{\gamma^{\prime}}$ are not empty. Otherwise, we set $N(\vec{d}, \vec{e}):=0$. Choosing $\tau_{2}$ such that $\sum_{\vec{d} \in \mathcal{E}(\Lambda) ;[\vec{d}] \neq \vec{h}} N(\vec{d}, \vec{e}) \leq \tau_{2}$ for any $\vec{e} \in \mathcal{E}(\Lambda)$, then

$$
\begin{aligned}
\|\sigma-v\|_{l^{2}(\mathcal{E}(\Lambda))}^{2}= & \sum_{\vec{d}, \vec{e} \in \mathcal{E}(\Lambda) ;[\vec{d}],[\vec{e}] \neq \vec{h}} \frac{N(\vec{d}, \vec{e}) a(\vec{d}) \overline{a(\vec{e})}}{\left\|s_{\lambda}\right\|_{L^{2}((0,1))}^{2}} \\
& \leq \sum_{D H_{\mathcal{E}(\Lambda)}(\Phi)} \tilde{\psi}_{\vec{d}},\left.P_{D H_{\mathcal{E}(\Lambda)}(\Phi)} \tilde{\psi}_{\vec{e}}\right|_{L^{2}(\mathcal{E}(\Lambda))}|a(\vec{d})||a(\vec{e})|\left\|\zeta_{\bullet}, \vec{d}\right\|_{l^{2}(\mathcal{E}(\Lambda))} \\
& \sum_{\vec{d}, \vec{e} \in \mathcal{E}(\Lambda) ;[\vec{d}],[\vec{e}] \neq \vec{h}}\left\|\zeta_{\bullet, \vec{e}}\right\|_{l^{2}(\mathcal{E}(\Lambda))} N(\vec{d}, \vec{e}) \\
\leq & \frac{6 \tau_{2}}{\left.\left|1-e^{-\left.i \Phi\right|^{2}} \sum_{\vec{e} \in \mathcal{E}(\Lambda) ;[\vec{e}] \neq \vec{h}}\right| a(\vec{e})\right|^{2}}
\end{aligned}
$$

\subsection{Absolutely continuous spectrum for rational flux quanta}

Lemma 8.15 For $\frac{\Phi}{2 \pi}=\frac{p}{q} \in \mathbb{Q}$, the spectrum of $H^{B}$ away from the Dirichlet spectrum is absolutely continuous and has possibly touching, but 


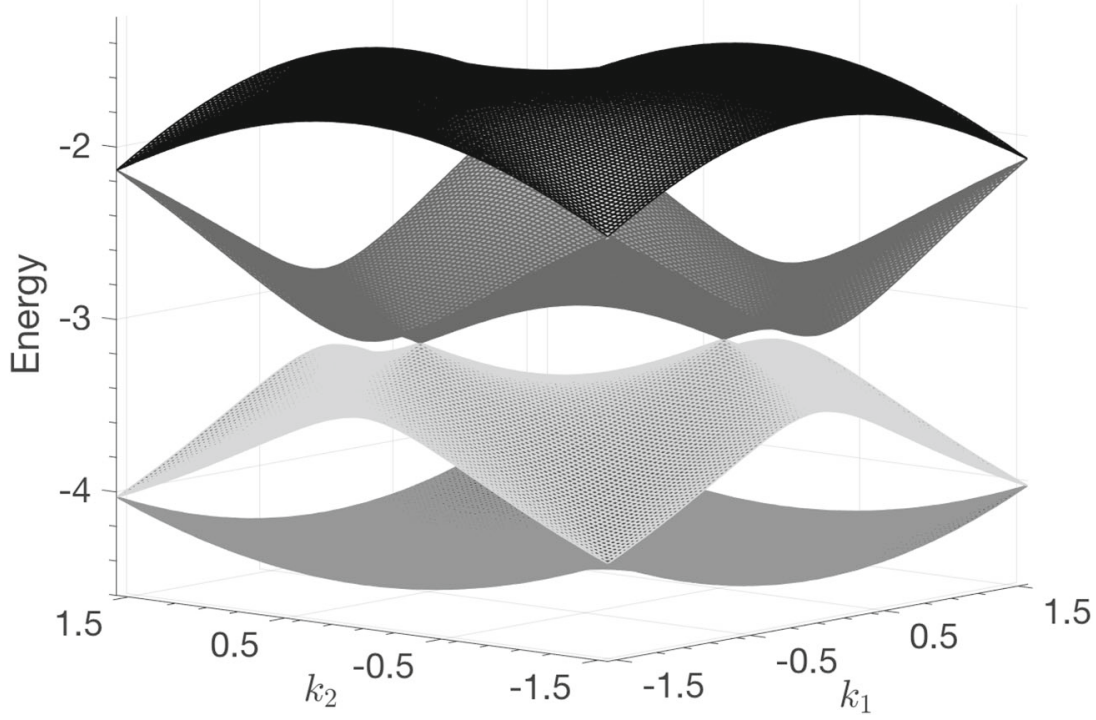

Fig. 9 Touching bands for $\frac{\Phi}{2 \pi}=\frac{1}{2}$ on the first Hill band of a Schrödinger operator with Mathieu potential $V(t)=20 \cos (2 \pi t)$. Different bands are differently colored

non-overlapping band structure. An interval $I \subset[-1,1]$ is a band of $Q_{\Lambda}(\Phi)$ if and only if its pre-image under $\Delta$, on each fixed band of the Hill operator, is a band of $H^{B}$.

Proof That the bands of $Q_{\Lambda}(\Phi)$ do not overlap is shown in Section 6 of [28]. Thus, the unique correspondence between bands of $Q_{\Lambda}(\Phi)$ and $H^{B}$, following from the unitary equivalence (7.14), shows that the non-overlapping of bands holds true for $H^{B}$ as well.

Remark 10 For $\frac{\Phi}{2 \pi}=\frac{1}{2}$ the spectral bands of $Q_{\Lambda}(\Phi)$ are touching and given by [28]

$$
\left[-\sqrt{\frac{2}{3}},-\sqrt{\frac{1}{3}}\right],\left[-\sqrt{\frac{1}{3}}, 0\right],\left[0, \sqrt{\frac{1}{3}}\right] \text {, and }\left[\sqrt{\frac{1}{3}}, \sqrt{\frac{2}{3}}\right] .
$$

Thus, by Lemma 8.15 the bands of $H^{B}$ on each Hill band are touching as well, see Fig. 9. Bands belonging to different Hill bands do, as a rule for $\Phi \in(0,2 \pi)$, not touch by Lemma 8.1 .

In the case of $\frac{\Phi}{2 \pi}=\frac{1}{3}$ however, only the bands at the Dirac points touch, see also Fig. 10. The touching at the Dirac points is always satisfied by Lemma 8.2. 


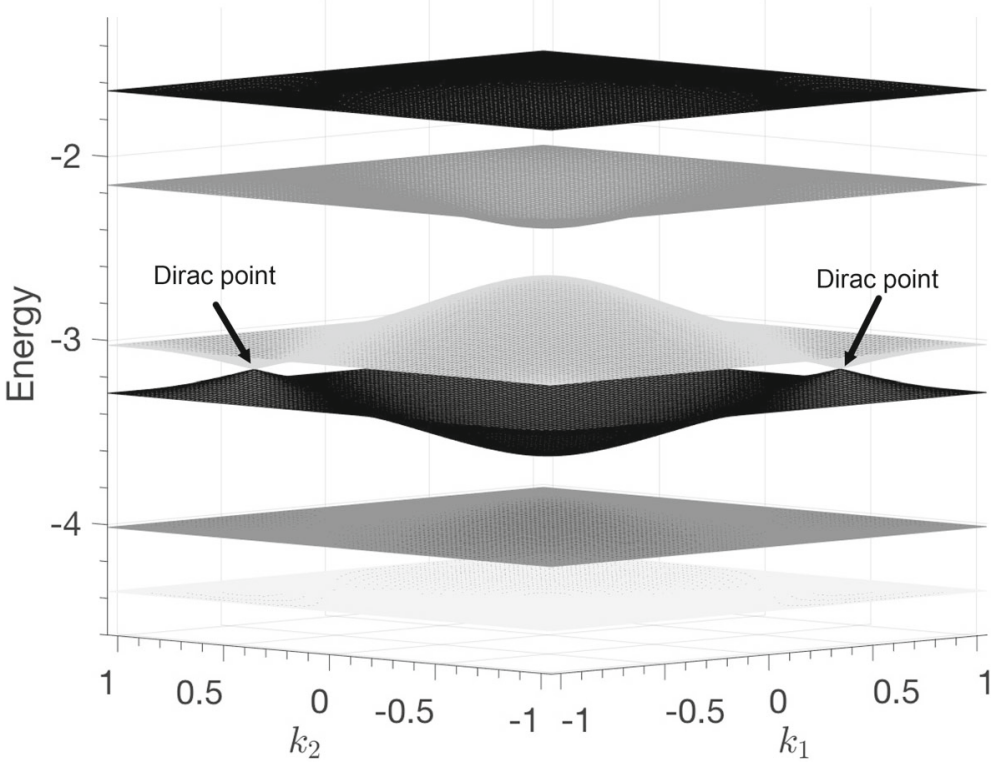

Fig. 10 Only the third and fourth band touch at the Dirac points for $\frac{\Phi}{2 \pi}=\frac{1}{3}$ on the first Hill band of a Schrödinger operator with Mathieu potential $V(t)=20 \cos (2 \pi t)$. Different bands are differently colored

\subsection{Singular continuous Cantor spectrum for irrational flux quanta}

Proof By Lemma 4.2, the spectrum of $Q_{\Lambda}(\Phi)$ for irrational $\frac{\Phi}{2 \pi}$ is a Cantor set of measure zero. Thus, the pullback of $\sigma\left(Q_{\Lambda}\right)$ by $\left.\Delta\right|_{\text {int }\left(B_{n}\right)}$ is still a Cantor set of zero measure that coincides with $\sigma\left(H^{B}\right) \backslash \sigma\left(H^{D}\right)$. Therefore, the absolutely continuous spectrum of $H^{B}$ has to be empty. The Cantor spectrum part of (3) of Theorem 1, and (1) of Theorem 2 then follows from (4) of Lemma 8.2.

\subsection{Proofs of Theorems 1-4}

This section serves as an index to the proofs of our main theorems that are distributed in different sections throughout the paper.

\section{Proof of Theorem 1}

(1). Follows from (1) of Lemma 8.7.

(2). Combine (3) of Lemma 8.2 with Lemma 8.4.

(3). This is proved in Sects. 8.3 and 8.4 (Fig. 11).

(4). Follows from (4) of Lemma 8.2.

(5). This is Lemma 8.3. 


\section{Hofstadter butterfly-Graphene}

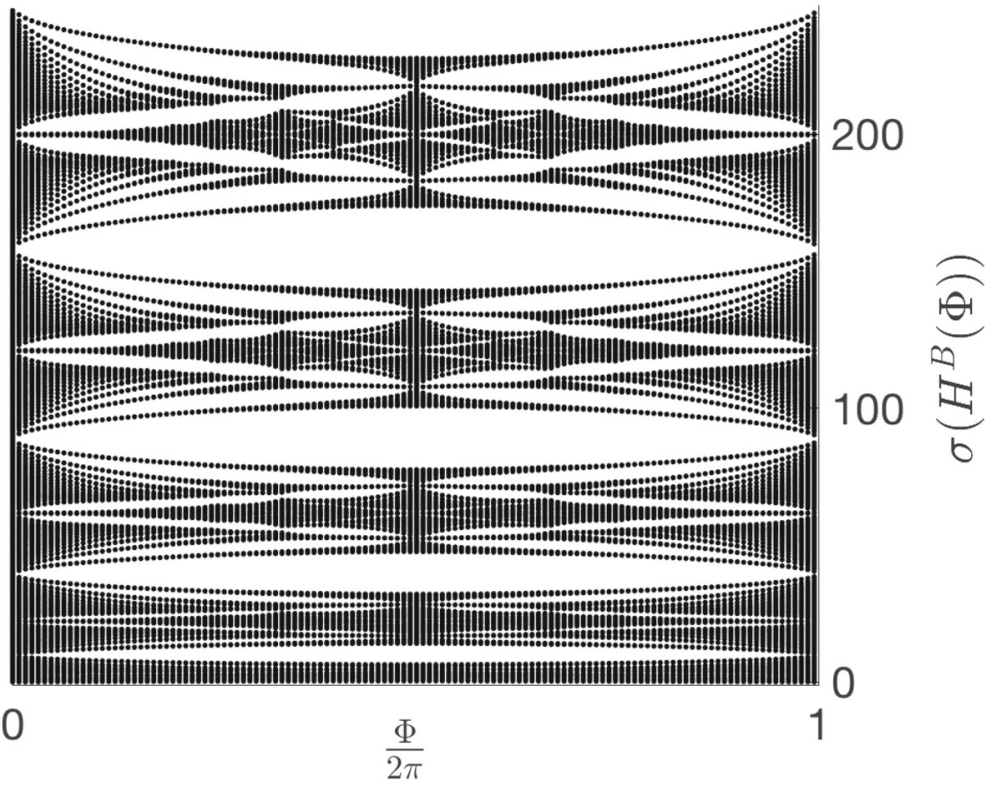

Fig. 11 The Hofstadter butterfly for $H^{B}$ with $V=0$ on the first five Hill bands $B_{k}=\left[\pi^{2}(k-\right.$ $\left.1)^{2}, \pi^{2} k^{2}\right]$ for $k \in\{1, . ., 5\}$ and magnetic flux quanta $\frac{\Phi}{2 \pi}=\frac{p}{q} \in[0,1]$ with $q \leq 50$

\section{Proof of Theorem 2}

This is proved in Sects. 8.3 and 8.4.

\section{Proof of Theorem 3}

This is proved in Lemmas 4.2, 4.3 and 4.4.

\section{Proof of Theorem 4}

This is proved in Lemma 8.10 and Theorem 10.

Acknowledgements This research was partially supported by the NSF DMS-1401204 and DMS-1901462. Support by the EPSRC grant EP/L016516/1 for the University of Cambridge CDT, the CCA is gratefully acknowledged (S.B.). R.H. would like to thank the Institute for Advanced Study, Princeton, for its hospitality during the 2017-2018 academic year. R.H. was supported in part by NSF DMS-1638352 and NSF-DMS-1800689.

Open Access This article is distributed under the terms of the Creative Commons Attribution 4.0 International License (http://creativecommons.org/licenses/by/4.0/), which permits unrestricted use, distribution, and reproduction in any medium, provided you give appropriate credit 
to the original author(s) and the source, provide a link to the Creative Commons license, and indicate if changes were made.

\section{Appendix A. Proof of Proposition 6.4}

The proof of this result is very similar to that for the almost Mathieu operator and the extended Harper's model. We will present it briefly here for completeness. Readers could refer to Theorem 3.2 (together with its proof in "Appendix 2") of [7] for a more detailed discussion.

Let $D^{\lambda}$ be defined as in (2.49), in which $v(\theta)=2 \cos 2 \pi \theta$ and $c(\theta)=$ $1+e^{-2 \pi i \theta}$, hence

$$
D^{\lambda}(\theta)=\left(\begin{array}{cc}
\lambda-e^{2 \pi i \theta}-e^{-2 \pi i \theta} & -1-e^{2 \pi i\left(\theta-\frac{\Phi}{2 \pi}\right)} \\
1+e^{-2 \pi i \theta} & 0
\end{array}\right) .
$$

Let us complexify $\theta$ and define $D_{\varepsilon}^{\lambda}$ for $\varepsilon \in \mathbb{R}$ as follows

$$
D_{\varepsilon}^{\lambda}(\theta):=D^{\lambda}(\theta+i \varepsilon)
$$

Let

$$
L\left(D_{\varepsilon}^{\lambda}, \Phi\right):=\lim _{n \rightarrow \infty} \frac{1}{n} \int_{\mathbb{T}_{1}} \log \left\|\prod_{j=n-1}^{0} D_{\varepsilon}^{\lambda}\left(\theta+j \frac{\Phi}{2 \pi}\right)\right\| \mathrm{d} \theta
$$

be the complexified Lyapunov exponent. By Hardy's convexity theorem, see e.g. Theorem 1.6 in [16], $L\left(D_{\varepsilon}^{\lambda}, \Phi\right)$ is convex in $\varepsilon$.

Let

$$
\omega(\lambda, \Phi ; \varepsilon):=\frac{1}{2 \pi} \lim _{h \rightarrow 0_{+}} \frac{L\left(D_{\varepsilon+h}^{\lambda}, \Phi\right)-L\left(D_{\varepsilon}^{\lambda}, \Phi\right)}{h}
$$

be the right-derivative of the complexified Lyapunov exponent, which has been dubbed acceleration in [2].

By Theorem 1 of [35], since $\operatorname{det}\left(D^{\lambda}(\theta+i \varepsilon)\right) \neq 0$ for $\varepsilon \neq 0$, we have

$$
\omega(\lambda, \Phi ; \varepsilon) \in \mathbb{Z}, \text { for } \varepsilon \neq 0 \text {. }
$$

This is usually referred to as quantization of acceleration. 
One can also easily compute the following asymptotic behaviour

$$
\begin{aligned}
& D_{\varepsilon}^{\lambda}(\theta)=\left(\begin{array}{cc}
-e^{2 \pi \varepsilon} & 0 \\
e^{2 \pi \varepsilon} & 0
\end{array}\right)+O(1), \quad \varepsilon \rightarrow \infty \\
& D_{\varepsilon}^{\lambda}(\theta)=\left(\begin{array}{cc}
-e^{-2 \pi \varepsilon} & -e^{-i \Phi} e^{-2 \pi \varepsilon} \\
0 & 0
\end{array}\right)+O(1), \quad \varepsilon \rightarrow-\infty,
\end{aligned}
$$

hence by (A.5),

$$
\left\{\begin{array}{l}
L\left(D_{\varepsilon}^{\lambda}, \Phi\right)=\varepsilon, \quad \varepsilon>\varepsilon_{0}>0, \\
L\left(D_{\varepsilon}^{\lambda}, \Phi\right)=-\varepsilon, \quad \varepsilon<-\varepsilon_{0} .
\end{array}\right.
$$

Hence convexity of $L\left(D_{\varepsilon}^{\lambda}, \Phi\right)$ and quantization of acceleration force either

- $L\left(D_{0}^{\lambda}, \Phi\right)=0$ or

- $L\left(D_{0}^{\lambda}, \Phi\right)>0$ with $\omega(0, \Phi ; \varepsilon)=0$.

By Theorem 1.2 of [8], the second case is equivalent to $\left(\frac{\Phi}{2 \pi}, D_{0}^{\lambda}\right)$ inducing a dominated splitting. This is equivalent to $\lambda \notin \Sigma_{\Phi}$, by [43].

Finally note that we always have

$$
L(\lambda, \Phi)=L\left(D_{0}^{\lambda}, \Phi\right)-\int_{\mathbb{T}_{1}} \log \left|1+e^{-2 \pi i \theta}\right| \mathrm{d} \theta=L\left(D_{0}^{\lambda}, \Phi\right)
$$

Hence $L(\lambda, \Phi)=0$ if and only if $\lambda \in \Sigma_{\Phi}$.

\section{Appendix B. Proof of Lemma 6.7}

Assume $\theta=\frac{1}{2}+k_{0} \frac{p}{q}$. Let $\left.\left(H_{2 \pi p / q, \theta}\right)\right|_{[0, k-1]}$ be the restriction of $H_{2 \pi p / q, \theta}$ onto interval $[0, k-1]$ with Dirichlet boundary condition. Let $P_{k}(\theta)=$ $\operatorname{det}\left(\lambda-\left.\left(H_{2 \pi p / q, \theta}\right)\right|_{[0, k-1]}\right)$ be the determinant of this $k \times k$ matrix. One can prove by induction (in $k$ ) that the following holds

$$
D_{k}^{\lambda}(\theta)=\left(\begin{array}{cc}
P_{k}(\theta) & -\overline{c\left(\theta-\frac{p}{q}\right)} P_{k-1}\left(\theta+\frac{p}{q}\right) \\
c\left(\theta+(k-1) \frac{p}{q}\right) P_{k-1}(\theta) & -\overline{c\left(\theta-\frac{p}{q}\right)} c\left(\theta+(k-1) \frac{p}{q}\right) P_{k-2}\left(\theta+\frac{p}{q}\right)
\end{array}\right) .
$$

Thus

$$
\operatorname{tr}\left(D_{q}^{\lambda}(\theta)\right)=P_{q}(\theta)-\left|c\left(\theta-\frac{p}{q}\right)\right|^{2} P_{q-2}\left(\theta+\frac{p}{q}\right) .
$$


It then suffices to note that

$$
\left\{\begin{array}{l}
\operatorname{tr}\left(D_{q}^{\lambda}\left(\theta-\left(k_{0}-1\right) \frac{p}{q}\right)\right)=\operatorname{tr}\left(D_{q}^{\lambda}(\theta)\right), \\
c\left(\theta-k_{0} \frac{p}{q}\right)=0, \\
\left(H_{\left.\frac{2 \pi p}{q}, \theta-\left(k_{0}-1\right) \frac{p}{q}\right)\left.\right|_{[0, q-1]}=M_{q} .} .\right.
\end{array}\right.
$$

\section{Appendix C. 1/2-Hölder continuity of spectra of Jacobi matrices}

\section{Proof of Lemma 6.2}

We will prove the following general result for quasi-periodic Jacobi matrices. Let $H_{\alpha, \theta} \in \mathcal{L}\left(l^{2}(\mathbb{Z})\right)$ be defined as

$$
\left(H_{\alpha, \theta} u\right)_{n}=c(\theta+n \alpha) u_{n+1}+\overline{c(\theta+(n-1) \alpha)} u_{n-1}+v(\theta+n \alpha) u_{n} .
$$

Let $\sigma_{\alpha}:=\cup_{\theta \in \mathbb{T}_{1}} \sigma\left(H_{\alpha, \theta}\right)$.

Lemma C.1 Let $c(\cdot), v(\cdot) \in C^{1}\left(\mathbb{T}_{1}, \mathbb{C}\right)$. There exist constants $\tilde{C}(c, v), C$ $(c, v)>0$ such that if $\lambda \in \sigma_{\alpha}$ and $\alpha^{\prime} \in \mathbb{T}_{1}$ is such that $\left|\alpha-\alpha^{\prime}\right|<\tilde{C}(c, v)$, then there is a $\lambda^{\prime} \in \sigma_{\alpha^{\prime}}$ such that

$$
\left|\lambda-\lambda^{\prime}\right| \leq C(c, v)\left|\alpha-\alpha^{\prime}\right|^{\frac{1}{2}}
$$

Lemma 6.2 follows from Lemma C.1 by taking $\Phi=2 \pi \alpha$ and $\Phi^{\prime}=2 \pi \alpha^{\prime}$. Lemma C.1 is in turn the argument of [9] adapted to the Jacobi setting.

\section{Proof of Lemma C.1}

Let $L \geq 1$ be given. There exists $\phi_{L} \in l^{2}(\mathbb{Z})$ and $\theta$ such that

$$
\left\|\left(H_{\alpha, \theta}-\lambda\right) \phi_{L}\right\| \leq \frac{1}{L}\left\|\phi_{L}\right\| .
$$

Let $\eta_{j, L}$ be the test function centered at $j$,

$$
\eta_{j, L}(n)= \begin{cases}(1-|n-j| / L), & |n-j| \leq L \\ 0, & |n-j| \geq L\end{cases}
$$

Then for large $L$,

$$
\sum_{j}\left(\eta_{j, L}(n)\right)^{2}=1+\frac{(L-1)(2 L-1)}{3 L} \equiv a_{L} .
$$


is independent of $n$. Clearly,

$$
\begin{aligned}
\sum_{j}\left\|\eta_{j, L}\left(H_{\alpha, \theta}-\lambda\right) \phi_{L}\right\|^{2} & =a_{L}\left\|\left(H_{\alpha, \theta}-\lambda\right) \phi_{L}\right\|^{2} \\
& \leq \frac{a_{L}}{L^{2}}\left\|\phi_{L}\right\|^{2}=\frac{1}{L^{2}} \sum_{j}\left\|\eta_{j, L} \phi_{L}\right\|^{2} .
\end{aligned}
$$

Since $\|u+v\|^{2} \leq 2\|v\|^{2}+2\|u\|^{2}$, by (C.4), we get

$$
\begin{aligned}
\sum_{j}\left\|\left(H_{\alpha, \theta}-\lambda\right) \eta_{j, L} \phi_{L}\right\|^{2} \leq & 2 \sum_{j}\left\|\eta_{j, L}\left(H_{\alpha, \theta}-\lambda\right) \phi_{L}\right\|^{2} \\
& +2 \sum_{j}\left\|\left[\eta_{j, L}, H_{\alpha, \theta}\right] \phi_{L}\right\|^{2} \\
\leq & \frac{2}{L^{2}} \sum_{j}\left\|\eta_{j, L} \phi_{L}\right\|^{2}+2 \sum_{j}\left\|\left[\eta_{j, L}, H_{\alpha, \theta}\right] \phi_{L}\right\|^{2},
\end{aligned}
$$

where $\left[\eta_{j, L}, H_{\alpha, \theta}\right]=\eta_{j, L} H_{\alpha, \theta}-H_{\alpha, \theta} \eta_{j, L}$ is the commutator. Note that

$$
\begin{aligned}
\left(\left[\eta_{j, l}, H_{\alpha, \theta}\right] \phi\right)_{n}=c(\theta+n \alpha)\left(\eta_{j, L}(n)\right. & \left.-\eta_{j, L}(n+1)\right) \phi_{n+1} \\
& +\overline{c(\theta+(n-1) \alpha)}\left(\eta_{j, L}(n)\right. \\
& \left.-\eta_{j, L}(n-1)\right) \phi_{n-1},
\end{aligned}
$$

which implies

$$
\sum_{j}\left\|\left[\eta_{j, L}, H_{\alpha, \theta}\right] \phi_{L}\right\|^{2} \leq \frac{8\|c\|_{\infty}^{2}}{L}\left\|\phi_{L}\right\|^{2} \leq \frac{8\|c\|_{\infty}^{2}}{L a_{L}} \sum_{j}\left\|\eta_{j, L} \phi_{L}\right\|^{2} .
$$

Combining this with (C.5) and taking into account that $a_{L} \sim \frac{2}{3} L$, we get

$$
\sum_{j}\left\|\left(H_{\alpha, \theta}-\lambda\right) \eta_{j, L} \phi_{L}\right\|^{2} \leq \frac{2+25\|c\|_{\infty}^{2}}{L^{2}} \sum_{j}\left\|\eta_{j, L} \phi_{L}\right\|^{2}
$$

for $L>L_{0}$. Hence for certain $j, \eta_{j, L} \phi_{L} \neq 0$ and

$$
\left\|\left(H_{\alpha, \theta}-\lambda\right) \eta_{j, L} \phi_{L}\right\| \leq \frac{\left(2+25\|c\|_{\infty}^{2}\right)^{\frac{1}{2}}}{L}\left\|\eta_{j, L} \phi_{L}\right\| .
$$


Given $\alpha^{\prime}$ near $\alpha$, choose $\theta^{\prime}$ such that

$$
\theta+j \alpha=\theta^{\prime}+j \alpha^{\prime}
$$

Then on $\operatorname{supp}\left(\eta_{j, L} \phi_{\varepsilon}\right)$,

$$
\left|f(\theta+n \alpha)-f\left(\theta^{\prime}+n \alpha^{\prime}\right)\right| \leq L\left\|f^{\prime}\right\|_{\infty}\left|\alpha-\alpha^{\prime}\right|,
$$

holds for $f=c, v$. Thus, by (C.6) and (C.7),

$$
\left\|\left(H_{\alpha^{\prime}, \theta^{\prime}}-\lambda\right) \eta_{j, L} \phi_{L}\right\| \leq C_{1}(c, v)\left\|\eta_{j, L} \phi_{L}\right\|
$$

where

$$
C_{1}(c, v)=\frac{\left(2+25\|c\|_{\infty}^{2}\right)^{\frac{1}{2}}}{L}+\left(6\left\|c^{\prime}\right\|_{\infty}^{2}+3\left\|v^{\prime}\right\|_{\infty}^{2}\right)^{\frac{1}{2}} L\left|\alpha-\alpha^{\prime}\right| .
$$

Finally, taking

$$
L=C_{2}(c, v)\left|\alpha-\alpha^{\prime}\right|^{-\frac{1}{2}}>L_{0},
$$

we get

$$
\left\|\left(H_{\alpha^{\prime}, \theta^{\prime}}-\lambda\right) \eta_{j, L} \phi_{L}\right\| \leq C(c, v)\left|\alpha-\alpha^{\prime}\right|^{\frac{1}{2}}\left\|\eta_{j, L} \phi_{L}\right\| .
$$

\section{References}

1. Avila, A.: On point spectrum with critical coupling (preprint). www.impa.br/ avila/

2. Avila, A.: Global theory of one-frequency Schrödinger operators. Acta Math. 215, 1-54 (2015)

3. Azbel, M.: Energy spectrum of a conduction electron in a magnetic field. Sov. Phys. JETP 19(3), 634-645 (1964)

4. Agazzi, A., Eckmann, J.-P., Graf, G.M.: The colored Hofstadter butterfly for the Honeycomb lattice. J. Stat. Phys. 156(3), 417-426 (2014)

5. Avila, A., Jitomirskaya, S.: The Ten Martini problem. Ann. Math. 170(1), 303-342 (2009)

6. Avila, A., Jitomirskaya, S.: Almost localization and almost reducibility. J. Eur. Math. Soc. 12(1), 93-131 (2010)

7. Avila, A., Jitomirskaya, S., Marx, C.: Spectral theory of extended Harper's model and a question by Erdôs and Szekeres. Invent. Math. 210(1), 283-339 (2017)

8. Avila, A., Jitomirskaya, S., Sadel, C.: Complex one-frequency cocycles. J. Eur. Math. Soc. 16(9), 1915-1935 (2014)

9. Avron, J., v Mouche, P., Simon, B.: On the measure of the spectrum for the almost Mathieu operator. Commun. Math. Phys. 132(1), 103-118 (1990) 
10. Aizenman, M., Warzel, S.: Resonant delocalization for random Schrödinger operators on tree graphs. J. Eur. Math. Soc. 15, 1167-1222 (2013)

11. Brüning, J., Geyler, V., Pankrashkin, K.: Cantor and band spectra for periodic quantum graphs with magnetic fields. Commun. Math. Phys. 269(1), 87-105 (2007)

12. Becker, S., Han, R.: In preparation

13. Becker, S., Zworski, M.: Magnetic Oscillations in a Model of Graphene. Commun. Math. Phys. 367(3), 941-989 (2019)

14. Becker, S., Han, R., Jitomirskaya, S., Zworski, M.: In preparation

15. Chen, X., Wallbank, A., Patel, A., Mucha-Kruczynski, M., McCann, E., Fal'ko, V.: Dirac edges of fractal magnetic minibands in graphene with hexagonal moiré superlattices. Phys. Rev. B 89(7), 075401 (2014)

16. Duren, P.: Theory of Hp Spaces, Pure and Applied Mathematics, vol. 38. Academic Press, New York (1970)

17. Dean, C.R., Wang, L., Maher, P., Forsythe, C., Ghahari, F., Gao, Y., Katoch, J., Ishigami, M., Moon, P., Koshino, M., Taniguchi, T., Watanabe, K., Shepard, K.L., Hone, J., Kim, P.: Hofstadter's butterfly in moire superlattices: a fractal quantum Hall effect. Nature 497, 598-602 (2013)

18. Dombrowsky, J.: Quasitriangular matrices. Proc. Am. Math. Soc. 69, 95-96 (1978)

19. Exner, P., Vaata, D.: Cantor spectra of magnetic chain graphs. J. Phys. A Math. Theor. 50(16), 165201 (2017)

20. Fefferman, C., Weinstein, M.: Honeycomb lattice potentials and Dirac points. J. Am. Math. Soc. 25(4), 1169-1220 (2012)

21. Fefferman, C., Weinstein, M.: Wave packets in honeycomb structures and two-dimensional Dirac equations. Commun. Math. Phys. 326, 251-286 (2014)

22. Fefferman, C., Lee-Thorp, M.J.P., Weinstein, M.: Honeycomb Schroedinger operators in the strong binding regime. Commun. Pure Appl. Math. 71, 6 (2018)

23. Garcia-C., H., Gaggero-S., L., Díaz-G., D.S., Sotolongo-C., O., Rodríguez-V., I.: Selfsimilar conductance patterns in graphene Cantor-like structures. Sci. Rep. 7(1), 617 (2017)

24. Gomes, K., Mar, W., Ko, W., Guinea, F., Manoharan, H.: Designer Dirac fermions and topological phases in molecular graphene. Nature 483(7389), 306-310 (2012)

25. Ponomarenko, L., Gorbachev, R., Yu, G., Elias, D., Jalil, R., Patel, A., Mishchenko, A., Mayorov, A., Woods, C., Wallbank, J., Mucha-Kruczynski, M., Piot, B., Potemski, M., Grigorieva, I., Novoselov, K., Guinea, F., Fal'ko, V., Geim, A.: Cloning of Dirac fermions in graphene superlattices. Nature 497, 594-597 (2013)

26. Gérard, C., Nier, F.: The Mourre theory for analytically fibered operators. J. Funct. Anal. 152(1), 202-219 (1998)

27. Guinea, F., et al.: Strain-induced pseudo-magnetic fields greater than 300 tesla in graphene nanobubbles. Science 329(5991), 544-547 (2010)

28. Helffer, B., Kerdelhué, P., Royo-Letelier, J.: Chambers's formula for the graphene and the Hou model. Ann. Henri Poincaré 17(4), 795-818 (2016)

29. Han, R.: Dry Ten Martini problem for the non-self-dual extended Harper's model. Trans. Amer. Math. Soc. 370, 197-217 (2018)

30. Han, R.: Absence of point spectrum for the self-dual extended Harper's model. Int. Math. Res. Not. 2018(9), 801-2809 (2018)

31. Hofstadter, D.: Energy levels and wave functions of Bloch electrons in rational and irrational magnetic fields. Phys. Rev. B 14(6), 2239-2249 (1976)

32. Jaksic, V., Last, Y.: Surface states and spectra. Commun. Math. Phys. 218, 459-477 (2001)

33. Jitomirskaya, S., Krasovsky, I.: Critical almost Mathieu operator: hidden singularity, gap continuity, and the Hausdorff dimension of the spectrum (preprint) (2019)

34. Jitomirskaya, S., Marx, C.: Analytic quasi-perodic cocycles with singularities and the Lyapunov exponent of extended Harper's model. Commun. Math. Phys. 316(1), 237-267 (2012) 
35. Jitomirskaya, S., Marx, C.: Erratum to: Analytic quasi-perodic cocycles with singularities and the Lyapunov exponent of extended Harper's model. Commun. Math. Phys. 317, 269271 (2013)

36. Kuchment, P.: Quantum graphs: II. Some spectral properties of quantum and combinatorial graphs. J. Phys. A Math. Gen. 38(22), 4887 (2005)

37. Kuchment, P.: An overview of periodic elliptic operators. Bull. Am. Math. Soc. 53(3), 343-414 (2016)

38. Kato, T.: Perturbation Theory for Linear Operators. Springer, Berlin (1995)

39. Kuchment, P., Post, O.: On the spectra of carbon nano-structures. Commun. Math. Phys. 275(3), 805-82 (2007)

40. Kerdelhué, P., Royo-Letelier, J.: On the low lying spectrum of the magnetic Schrödinger operator with kagome periodicity. Rev. Math. Phys. 26(10), 1450020 (2014)

41. Kostrykin, V., Schrader, R.: Quantum wires with magnetic fluxes. Commun. Math. Phys. 237(1), 161-179 (2003)

42. Last, Y.: Zero measure spectrum for the almost Mathieu operator. Commun. Math. Phys. 164, 421-432 (1994)

43. Marx, C.: Dominated splittings and the spectrum of almost periodic Jacobi operators. Nonlinearity 27, 3059-3072 (2014)

44. Novoselov, K.: Nobel lecture: graphene: materials in the flatland. Rev. Mod. Phys. 83, 837-849 (2011)

45. Puig, J.: Cantor spectrum for the almost Mathieu operator. Commun. Math. Phys. 244(2), 297-309 (2004)

46. Pankrashkin, K.: Spectra of Schrödinger operators on equilateral quantum graphs. Lett. Math. Phys. 77(2), 139-154 (2006)

47. Pankrashkin, K.: An example of unitary equivalence between self-adjoint extensions and their parameters. J. Funct. Anal. 265(2013), 2910-2936, 640-655 (2013)

48. Pankrashkin, K.: Unitary dimension reduction for a class of self-adjoint extensions with applications to graph-like structures. J. Math. Anal. Appl. 396, 640-655 (2014)

49. Reed, M., Simon, B.: Analysis of Operators, Methods of Modern Mathematical Physics, vol. IV. Elsevier, Amsterdam (1978)

50. Schmüdgen, K.: Unbounded Self-adjoint Operators on Hilbert Space. Graduate Texts in Mathematics. Springer, Berlin (2012)

51. Thomas, L.: Time dependent approach to scattering from impurities in a crystal. Commun. Math. Phys. 33(4), 335-343 (1973)

52. Teschl, G.: Jacobi Operators and Completely Integrable Nonlinear Lattices, Mathematical Surveys and Monographs, vol. 72. Amer. Math. Soc, Providence (2000)

53. Toda, M.: Theory of Nonlinear Lattices. Springer, Berlin (1981)

54. Zak, J.: Magnetic translation group. Phys. Rev. 134(6A), A1602 (1964)

55. Zhang, Y., Tan, Y.-W., Stormer, H.-L., Kim, P.: Experimental observation of the quantum Hall effect and Berry's phase in graphene. Nature 438(7065), 201-204 (2005)

Publisher's Note Springer Nature remains neutral with regard to jurisdictional claims in published maps and institutional affiliations. 